\title{
S3-Leitlinie der Deutschen Gesellschaft für Verdauungs- und Stoffwechsel- krankheiten und der Deutschen Gesellschaft für Viszeralchirurgie zur Diagnostik und Behandlung von Gallensteinen AWMF-Register-Nr. $021 / 008$
}

S3-Guidelines for Diagnosis and Treatment of Gallstones. German Society for Digestive and Metabolic Diseases and German Society for Surgery of the Alimentary Tract. AWMF Registry 021/008

Authors

Affiliations
F. Lammert ${ }^{1}$, M. W. Neubrand ${ }^{2}$, R. Bittner ${ }^{3}$, H. Feussner ${ }^{4}$, L. Greiner ${ }^{5}$, F. Hagenmüller ${ }^{6}$, K. H. Kiehne ${ }^{7}$, K. Ludwig ${ }^{8}$, H. Neuhaus ${ }^{9}$, G. Paumgartner ${ }^{10}$, J. F. Riemann ${ }^{11}$, T. Sauerbruch ${ }^{1}$ für die Teilnehmer der Konsensuskonferenz ${ }^{*}$

Die Institutsangaben sind am Ende des Beitrags gelistet.
Schlüsselwörter

- Gallenblasensteine

- Gallengangssteine

- Cholelithiasis

- Cholezystektomie

Key words

- gallstones

- bile duct stones

- cholelithiasis

- cholecystectomy received 26.3.2007

accepted $\quad 16.7 .2007$

\section{Bibliography}

DOI $10.1055 / \mathrm{s}-2007-963437$

Z Gastroenterol 2007; 45:

971-1001 @ Karl Demeter

Verlag im Georg Thieme Verlag

KG Stuttgart · New York .

ISSN 0044-2771

\section{Correspondence}

\section{Prof. Dr. Frank Lammert}

Prof. Dr. Tilman Sauerbruch

Medizinische Klinik und Poli-

klinik I, Rheinische Friedrich-

Wilhelms-Universität Bonn

Sigmund-Freud-Straße 25

53105 Bonn

Tel.: ++ 49/228/28715216

Fax: ++ 49/2 28/28714698

frank.lammert@ukb.uni-

bonn.de

\section{Zusammenfassung \\ $\nabla$}

Die Leitlinie zur Diagnostik und Therapie von Gallensteinen aktualisiert die Leitlinie der Deutschen Gesellschaft für Verdauungs- und Stoffwechselkrankheiten aus dem Jahr 2000. Die Leitlinie wurde interdisziplinär von Gastroenterologen und Chirurgen erstellt. Sie basiert auf den Empfehlungen der Arbeitsgemeinschaft der Wissenschaftlichen Medizinischen Fachgesellschaften für eine systematische evidenzbasierte Konsensus-Leitlinie der Entwicklungsstufe S3 und berücksichtigt die klinische Anwendbarkeit der Studienergebnisse. Zudem werden Präventionsmöglichkeiten beschrieben, Qualitätsindikatoren definiert, ökonomische Auswirkungen berücksichtigt und Vorschläge zur Verzahnung der ambulanten und stationären Versorgung von Patienten mit Gallensteinerkrankungen formuliert.

\section{Vorwort}

\section{$\nabla$}

15 - 20\% unserer Bevölkerung haben Gallensteine [1-4], und jährlich werden in Deutschland mehr als 190000 Cholezystektomien durchgeführt [5]. Das Gallensteinleiden ist damit nach der Refluxerkrankung die gastroenterologische Erkrankung, die die höchsten Kosten verursacht [6]. Die nachfolgende Leitlinie zur Diagnostik und Therapie dieser sehr häufigen Erkrankung aktualisiert die Leitlinie der Deutschen Gesellschaft für Verdauungs- und Stoffwechselkrankheiten (DGVS) aus dem Jahr 2000 [7]. Die neue Leitlinie wurde von der DGVS und der Deutschen Gesellschaft für Viszeralchirurgie (DGVC) gemeinsam entwickelt. Zielgruppe der Leitlinie sind alle praktizierenden Ärztinnen und Ärzte in Deutschland, vorrangig niedergelassene und in Krankenhäusern tätige Allgemeinmediziner, In-

\footnotetext{
* siehe Addendum A für vollständige Teilnehmerliste.
}

\section{Abstract \\ $\nabla$}

This guideline provides evidence-based key recommendations for diagnosis and therapy of gallstones and upgrades version 2000. It was developed by an interdisciplinary team of gastroenterologists, surgeons, radiologists, geneticists, external comparative quality assurance and patient support groups under the auspices of the German Society for Digestive and Metabolic Diseases and the German Society for Surgery of the Alimentary Tract. The guideline used structural S3 consensus-based methodology and includes statements on clinical practice, prevention, outcome analysis, and integration of outpatient and inpatient care for patients with gallstone diseases.

ternisten und Chirurgen, deren Kooperationspartner in Qualitätszirkeln, Praxisnetzen und Klinikverbünden sowie die Kostenträger. Darüber hinaus dient die Leitlinie zur Information von Patienten und Öffentlichkeit über die angemessene medizinische Vorgehensweise bei Gallensteinerkrankungen. Die Leitlinie ist eine systematisch entwickelte ärztliche Orientierungshilfe mit Handlungs- und Entscheidungsvorschlägen, von denen in begründeten Fällen abgewichen werden kann oder sogar muss. Die Entscheidung, ob einer bestimmten Empfehlung gefolgt wird, trifft der behandelnde Arzt unter Berücksichtigung der individuellen Situation seines Patienten.

\section{Methodisches Vorgehen (Leitlinienreport) \\ $\nabla$}

Die vorausgegangene Konsensuskonferenz hatte die Güte der Empfehlung hauptsächlich aus der 
Evidenzstärke abgeleitet [7]. Unberücksichtigt blieben bei diesem Vorgehen die Konsensusstärke und klinische Algorithmen. Die neue Leitlinie beruht auf den drei Grundpfeilern Evidenzstärke, Konsensusstärke und klinische Anwendbarkeit der Studienergebnisse. Wesentliche Kriterien der Leitlinie sind ihre interdisziplinäre Erstellung unter Einbeziehung von Patientenvertretern, die systematische Literaturrecherche mit der Beurteilung ihrer Qualität und ihrer Übertragbarkeit auf den klinischen Alltag sowie die interdisziplinäre Bewertung der Kernaussagen der Leitlinie nach den Kriterien der evidenzbasierten Medizin (EBM). Zudem wurden erstmals Qualitätsindikatoren definiert, ökonomische Auswirkungen berücksichtigt, Vorschläge zu Outcome-Analysen formuliert und Aussagen zur Verzahnung ambulanter und stationärer Versorgungsbereiche gemacht.

Die methodische Grundlage für die Leitlinie waren das Leitlinien-Manual der Arbeitsgemeinschaft der Wissenschaftlichen Medizinischen Fachgesellschaften (AWMF) und des Ärztlichen Zentrums für Qualität in der Medizin [8], das Deutsche Instrument zur methodischen Leitlinien-Bewertung (DELBI) [9] und die Methodik der DGVS-Konsensusempfehlungen für die Colitis ulcerosa [10]. Die Leitlinie folgt daher methodisch und organisatorisch den Empfehlungen der AWMF für eine evidenzbasierte Konsensus-Leitlinie der Entwicklungsstufe 3 (S3-Leitlinie mit allen Elementen systematischer Entwicklung). Zur weiteren Planung des Konsensusprozesses erfolgte eine Beratung durch die Ständige Kommission Leitlinien der AWMF (H.-K. Selbmann, I. Kopp).

\section{Organisatorischer Ablauf des Konsensusprozesses}

Der zeitliche Ablauf des gesamten Konsensusprozesses ist im Addendum B dargestellt. Nach Erteilung eines Auftrags an T. Sauerbruch und F. Lammert zur Aktualisierung der Leitlinie durch die Leitlinienkommission der DGVS wurde das Leitlinienprojekt am 22.7.2005 bei der AWMF angemeldet (AWMFRegister-Nr. 021/008). Ein Steering-Komitee (T. Sauerbruch, F. Lammert, M. Neubrand, R. Bittner) erstellte, basierend auf dem Teilnehmerkreis der früheren Konsensuskonferenz, eine Teilnehmerliste für insgesamt fünf Arbeitsgruppen (siehe Addendum D). Dabei erfolgte die Auswahl unter Berücksichtigung folgender Kriterien:

- Versorgungsebene: Niedergelassene, Krankenhaus der Grund-, Schwerpunkt- oder Maximalversorgung

- Schlüsseldisziplin: Innere Medizin/Gastroenterologie, Chirurgie/Viszeralchirurgie, Radiologie, Humangenetik

- Hierarchische Stufe: Assistenzärztin/-arzt, Oberärztin/-arzt, Chefarzt.

Die Teilnehmer wurden den folgenden Arbeitsgruppen zugeordnet (siehe Addendum D):

1. Prävention und konservative Therapie der Cholelithiasis

2. Diagnostik der Cholezysto- und Choledocholithiasis

3. Chirurgische Therapie der Cholezystolithiasis

4. Therapie der Choledocholithiasis und der biliären Pankreatitis

5. Qualitätssicherung.

Es erfolgte eine systematische Literaturrecherche in MEDLINE und der Cochrane Database of Systematic Reviews, wobei in MEDLINE nur deutsch- und englischsprachige Originalarbeiten ab 1999 berücksichtigt wurden. Literatur vor 1999 war bereits für die vorausgegangene Leitlinie [7] gesichtet worden. Die verwendeten Suchbegriffe finden sich im Addendum C. Die Literatur wurde allen Arbeitsgruppenmitgliedern als Volltext kopiert zur Verfügung gestellt.
Zur weiteren Vorbereitung der Konferenz wurde ein elektronischer Fragebogen erstellt, der per E-mail zirkuliert wurde. Die Fragebögen wurden vor Beginn der Konsensuskonferenz von $83 \%$ (44/53) der teilnehmenden Ärzte beantwortet; zu jeder Frage konnten ergänzende Kommentare gemacht werden. Die statistische Auswertung erfolgte mit Excel-Software, wobei für jede Frage die Konsensusstärke, errechnet nach dem prozentualen Anteil einer Antwortmöglichkeit (siehe Addendum A), ermittelt wurde.

Im Rahmen der eigentlichen Konsensuskonferenz am 7.4.2006 in Bonn (Teilnehmer siehe Addendum E) wurden am Vormittag durch die Arbeitsgruppen Vorschläge für die Konsensusempfehlungen vorbereitet. Diese basierten auf der statistischen Fragebogenauswertung und den gesammelten anonymisierten Kommentaren und Literaturergänzungen der Experten. Am Nachmittag wurde der Konsensusvorschlag jeder Arbeitsgruppe vorgetragen, im Plenum diskutiert und über die ggf. modifizierten Konsensusempfehlungen abgestimmt. Dabei wurden die Evidenzstärke (Literatur), die klinische Anwendbarkeit und die Ergebnisse des Fragebogens (nominaler Gruppenprozess) berücksichtigt. Die Abstimmung im Plenum hatte bei Diskrepanzen Vorrang vor der E-mail-basierten Fragebogenabstimmung.

Der Konsensus wurde entsprechend der aufgezeichneten Diskussion vom Steering-Komitee bearbeitet. Anschließend wurden die Kommentare zu den Leitlinien von den Vorsitzenden der Arbeitsgruppen (siehe Addendum D) erstellt und allen Teilnehmern der Leitlinienkonferenz zur Korrektur zugesandt. Das Manuskript wurde abschließend der Kommission für Leitlinien der DGVS vorgelegt und vor der Veröffentlichung durch unabhängige Experten aus dem medizinischen Bereich und Methodiker begutachtet (J.C. Hoffmann, Berlin; J.C. Arnold, Rotenburg).

Klassifikationen der Evidenzstärke, der Konsensusstärke, der Anwendbarkeit und der Empfehlungsstärke Die Klassifikationen der Evidenz- und Konsensusstärken sowie der Anwendbarkeit der Konsensusempfehlungen sind dem Addendum A zu entnehmen. Die Einteilungen basieren auf dem methodischen Vorgehen der DGVS-Leitlinie zur Colitis ulcerosa [10] und einer modifizierten Klassifikation des Ärztlichen Zentrums für Qualität in der Medizin, die den Empfehlungen der AWMF entspricht [8, 11, 12]. Empfehlungen setzen voraus, dass eine Studie und/oder eine Expertenmeinung auf die Zielpopulation der Leitlinie bzw. auf den einzelnen Patienten anwendbar sind. Diese klinische Anwendbarkeit hängt von der untersuchten Patientengruppe, den Fähigkeiten der Leistungserbringer und dem Ressourcenbedarf ab. Basierend auf den Eckpfeilern Evidenzstärke, Konsensusstärke und Anwendbarkeit wurde ein Algorithmus verwendet [10], der die Empfehlungsstärke in „sehr empfehlenswert“, „empfehlenswert“ und „optional“ einteilt. In vier Fällen wurde eine Empfehlungsstärke abweichend vom Algorithmus (Addendum A) höher eingestuft; die Begründungen für diese Abweichungen sind im zugehörigen Kommentar genannt.

\section{Visualisierung und Implementierung}

Die logische Handlungsstruktur der Leitlinie bzw. ihre Entscheidungsregeln wurden mithilfe der Methode knowledgeTools $^{\circledR}$ [13] überprüft und modelliert (K. Wuhrow, http:// www.knowledgetools.de/). Zudem wurde eine Visualisierung der Leitlinien in Form einer Baumstruktur entwickelt ( $\odot$ Abb.1). Der Arzt kann sich bei Diagnostik- und Therapie- 
entscheidungen an diesen visualisierten Algorithmen orientieren.

Der Leitlinientext wird in der Zeitschrift für Gastroenterologie veröffentlicht. Der Volltext mit methodischen Hinweisen und Evidenztabellen ist über die Homepage der DGVS (http:// www.dgvs.de/) und der AWMF (http://www.awmf-online.de/) frei verfügbar. Eine Kurzfassung der Leitlinie wird in weiteren Medien, u.a. im Manual „Rationale Diagnostik und Therapie in der Inneren Medizin“ der Deutschen Gesellschaft für Innere Medizin (DGIM) und des Berufsverbands Deutscher Internisten (BDI), veröffentlicht und ist über die digitale Version dieses Leitlinienwerks ebenfalls abrufbar (http://www1.us. elsevierhealth.com/BDI/). Informationen für Patienten werden über Selbsthilfegruppen (Deutsche Leberhilfe e.V.) verbreitet. Ein weiteres Element des Implementierungskonzeptes ist die Einbringung der Leitlinien in die Aus-, Weiter- und Fortbildung von Ärzten und anderen Gesundheitsberufen durch die Mitglieder des Steering-Komitees und die Teilnehmer der Konsensuskonferenz.

\section{Finanzierung}

Die Leitlinienerstellung wurde durch Mittel der DGVS unabhängig von Interessengruppen finanziert. Diese Mittel wurden auf ein vom Universitätsklinikum Bonn verwaltetes Konto überwiesen. Reise- und Sekretariatskosten wurden nach den für Hochschulen in NRW gültigen Richtlinien erstattet. Es wurden keine Industriemittel verwendet, und es erfolgte keine externe Beeinflussung der Konferenzteilnehmer und Leitlinienautoren in Bezug auf die Empfehlungen und Kommentare der Leitlinie.

Die Teilnehmer der Konsensuskonferenz erteilten in schriftlicher Form Auskunft zu möglichen finanziellen Interessenkonflikten und unterzeichneten eine Erklärung zur Unabhängigkeit von Interessenbindungen (siehe Addendum E). Es wurden konkrete Angaben zu Berater- und Gutachtertätigkeiten für Industrieunternehmen, finanziellen Zuwendungen und Besitz von Geschäftsanteilen der pharmazeutischen oder biotechnologischen Unternehmen sowie bezahlte Autorenschaft bei Artikeln im Auftrag pharmazeutischer oder biotechnologischer Unternehmen erbeten.

\section{Gültigkeit der Leitlinie}

Die Leitlinie ersetzt die bisherige Version [7] und ist bis zur nächsten Überarbeitung bzw. spätestens bis zum 31.12.2012 gültig. Sollten in dieser Zeit relevante neue Erkenntnisse zur Diagnostik und Therapie von Gallensteinen bekannt werden, wird die Leitlinie zu einem früheren Zeitpunkt revidiert. $\bullet$ Tab. 1 fasst Hinweise der Teilnehmer der Konsensuskonferenz zu notwendigen zukünftigen Forschungsfeldern zusammen. Anregungen und Kommentare werden an die korrespondierenden Autoren (F. Lammert, T. Sauerbruch) als Ansprechpartner für die Aktualisierung der Leitlinie erbeten.

\section{Danksagung}

Wir danken allen Teilnehmern der Konsensuskonferenz (siehe Addendum E) sowie den Mitarbeiterinnen und Mitarbeitern der Medizinischen Klinik und Poliklinik I des Universitätsklinikums Bonn (U. Henseler, U. Moser) und des Universitätsclubs Bonn e.V. (A. Helmer, S. Jöher, K. Stüber) für die Unterstützung bei der Durchführung der Konsensuskonferenz.
Tab. 1 Zukünftige Forschungsfelder (Auswahl)

Nach Ansicht der Teilnehmer der Konsensuskonferenz sollten zukünftig insbesondere die folgenden Forschungsfelder bearbeitet werden, um die Therapie von Gallensteinen weiter zu verbessern:

- Identifizierung genetischer und exogener lithogener Risikofaktoren sowie neuer Präventionsstrategien

- Kosten-Nutzen-Analysen bei natürlichem Verlauf bei stummen oder wenig symptomatischen Gallenblasensteinen im Vergleich zur laparoskopischen Cholezystektomie

- Versorgungsforschung zu Langzeitresultaten der Cholezystektomie und regional unterschiedlichen Eingriffshäufigkeiten

- Studien zum Risiko biliärer Koliken und Komplikationen von asymptomatischen Gallensteinen und Gallenblasen-Sludge

- Pathogenese und Prävention von (rezidivierenden) Gallengangssteinen, Studien zur bakteriellen Besiedlung und Entzündung der Gallenwege sowie zur Antibiotikaprophylaxe

- Versorgungsforschung zur Frage der einzeitigen intraoperativen Choledochusrevision versus therapeutisches Splitting

\section{Begriffsdefinitionen}

Akute Cholangitis: Das klinische Bild der akuten steinbedingten obstruktiven Cholangitis ist geprägt durch die Charcot-Trias: Fieber, Ikterus, Oberbauchschmerz. Insbesondere bei alten oder immuninkompetenten Patienten kann die entzündliche Komponente (Fieber, Entzündungsparameter, Schmerz) fehlen oder schwächer ausgeprägt sein [14-17].

Akute Cholezystitis: Die akute kalkulöse (lithogene) Cholezystitis wird durch biliäre Schmerzen, die länger als $6 \mathrm{~h}$ anhalten, Fieber bzw. Leukozytose und Gallenblasenwandödem (Ultraschall) in Kombination mit lokalem Druckschmerz (klinisches oder sonografisch-palpatorisches Murphy-Zeichen) definiert [18, 19].

Biliäre Pankreatitis: Ein biliärer Ursprung einer Pankreatitis kann angenommen werden, wenn Gallensteine in einem bildgebenden Verfahren erkannt werden und ein Transaminasenanstieg beobachtet wird [20-23].

Biliäre Schmerzen: Akut einsetzende, gut erinnerliche Schmerzen im Epigastrium oder rechten Oberbauch, die länger als 15 min, aber kürzer als $5 \mathrm{~h}$ anhalten [7, 24, 25].

Komplikationen der Cholezystolithiasis: Im vorliegenden Leitlinientext werden unter dem Begriff Komplikationen die akute Cholezystitis, die Gallenblasenperforation, die akute Cholangitis und die biliäre Pankreatitis, die die häufigsten klinisch relevanten Komplikationen darstellen, zusammengefasst.

Leitlinie: Systematisch unter Berücksichtigung der Strategien und Methoden der evidenzbasierten Medizin entwickelte, wissenschaftlich begründete und praxisorientierte Entscheidungshilfe für Ärzte, andere Gesundheitsberufe und Patienten über die angemessene Vorgehensweise bei speziellen Gesundheitsproblemen $[8,9]$.

Pankreatitis: Die Diagnose einer akuten Pankreatitis ist bei akuten Bauchschmerzen, kombiniert mit eindeutiger Erhöhung der Serumaktivitäten der Pankreasenzyme (zumeist initial mehr als das Dreifache der oberen Normgrenze) zu stellen [26].

Sentinel Event: Sehr seltene, schwerwiegende Komplikation, der in einer differenzierten Einzelfallanalyse nachgegangen werden soll. Ziel ist es, diese Komplikationen vollständig zu vermeiden.

Hinweise: Die Leitlinienempfehlungen sind fett, die Kommentare zu den einzelnen Empfehlungen kursiv gedruckt. Die Evidenzstärke, die Konsensusstärke und die Einstufung der Konsensusemp- 
fehlungen sind hinter diesen in Klammern angegeben (zur Erklärung siehe Addendum A).

\section{Gallenblasensteine}

Diagnostik bei Gallenblasensteinen

1. Charakteristische Symptome für Gallenblasensteine sind, abgesehen von Komplikationen, gut erinnerliche Schmerzattacken von mehr als 15 min Dauer im Epigastrium oder rechten Oberbauch, die auch in den Rücken und in die rechte Schulter ausstrahlen können; nicht selten besteht zudem Übelkeit, gelegentlich auch Erbrechen (III, starker Konsens, C).

Kommentar: Obwohl die Spezifität charakteristischer biliärer Symptome umstritten ist, gibt es derzeit keine bessere Definition [27]. Eine neuere Multicenterstudie aus Italien bestätigt das signifikant häufigere Vorkommen der o.a. Beschwerden bei Gallensteinträgern [25]. Eine dänische Prävalenzstudie [28] beobachtete bei Männern mit Gallensteinen am häufigsten Schmerzen im rechten Unterbauch. In einer kürzlich publizierten prospektiven Studie in Norwegen [29] waren die biliären Koliken bei der Mehrzahl der Steinpatienten mit funktionellen abdominellen Beschwerden assoziiert. Dyspepsie und Blähungen sind jedoch keine steintypischen Beschwerden, da sie mindestens gleich häufig bei steinfreien Personen oder Patienten mit Reizdarmsyndrom auftreten [28, 30, 31] und nach Cholezystektomie häufig persistieren [32]. Nach erstmaliger Symptomatik entwickelt mehr als die Hälfte der Patienten im weiteren Verlauf neuerliche Schmerzattacken [33-36]. Allerdings reduziert sich das Risiko nach 5 beschwerdefreien Jahren auf das eines asymptomatischen Steinträgers [34], so dass Patienten mit einer Kolik, die länger als 5 Jahre zurückliegt, wieder der Gruppe asymptomatischer Steinträger zugeordnet werden.

Charakteristische biliäre Symptome sind ein Indiz für ein erhöhtes Risiko, Komplikationen der Cholelithiasis zu entwickeln. Die jährliche Komplikationsrate beträgt nach einer erstmaligen Kolik 1 -3\%, beim asymptomatischen Steinträger jedoch nur 0,1-0,3\% [35, 36]. Dem Patienten mit symptomatischer Cholezystolithiasis wird daher nicht nur zur Verhinderung neuerlicher Schmerzattacken, sondern auch zur Verhinderung von Komplikationen zur Cholezystektomie geraten (siehe II.5).

2. Der Nachweis oder Ausschluss einer Cholezystolithiasis erfolgt primär durch die standardisierte transkutane B-ModeSonografie (Ila, starker Konsens, A).

Kommentar: Die Sonografie ist die Methode der Wahl für die Diagnostik von Gallenblasensteinen mit einer Sensitivität > 95\% [18]. Darüber hinaus kann die Sonografie einen Hinweis auf ein gleichzeitiges Gallenblasenkarzinom oder Gallenblasenpolypen geben. Die Untersuchung sollte standardisiert erfolgen. Dies erfordert den Einsatz der aktuellen Gerätetechnik durch erfahrene ärztliche Untersucher. Unabdingbar ist die komplette Darstellung der Gallenblase in variablen Schnittebenen und in zumindest zwei Patienten-Lagerungsvarianten, die um $90^{\circ}$ zueinander versetzt sind, um das typische gravitationsabhängige Migrationsverhalten auch kleinerer Steine zu erfassen. Die Gallenblasenübersichtsaufnahme oder die Computertomografie sind allenfalls zum Ausschluss oder Nachweis kalkhaltiger Steine sinnvoll. Die orale oder intravenöse Cholangiografie zur Diagnostik von Gallensteinen wird nicht mehr angewandt.
3. Die Sonografie ist in Kombination mit den klinischen Befunden die Methode der Wahl zum Nachweis oder Ausschluss der akuten Cholezystitis (Ib, starker Konsens, A).

Kommentar: Die Sonografie hat eine hohe Sensitivität von 94\% und eine Spezifität von 78\% für die Diagnose der akuten Cholezystitis $[18,19]$. Der isolierte sonografische Befund einer Gallenblasenwandverdickung ( $>4 \mathrm{~mm}$ ) ist nicht pathognomonisch für eine akute Cholezystitis. Eine Dreischichtung der Wand und ein echoarmer Flüssigkeitssaum um die Gallenblase weisen auf eine akute Cholezystitis hin; differenzialdiagnostisch kann eine Gallenblasenwandverdickung auch bei akuter Hepatitis, portaler Hypertension, Hypoproteinämie, Rechtsherzinsuffizienz oder Polyserositis auftreten [37, 38]. Charakteristisch für die akute Cholezystitis ist das sonografisch-palpatorische Murphy-Zeichen (fokaler Schmerz unter direktem Druck). Für dieses wird ein positiver prädiktiver Wert $>90 \%$ angegeben, der in Zusammenschau mit der Wandverdickung auf $>99 \%$ ansteigt [39]. Komplikationen wie die gedeckte oder die offene Perforation sind sonografisch gut nachweisbar bzw. auszuschließen. Die zusätzliche Darstellung durch ein weiteres Schnittbildverfahren kann in Einzelfällen sinnvoll sein. Die hepatobiliäre Funktionsszintigrafie ist in Deutschland zur Diagnostik der akuten Cholezystitis kein akzeptierter Standard.

4. Bei außergewöhnlicher klinischer Konstellation der Cholelithiasis (z. B. familiäre Häufung, Auftreten im Kindes- und Jugendalter, intrahepatische Steine, Assoziation mit Diarrhöen) sollte eine weitere ätiologische Abklärung erfolgen (z. B. hämolytische Anämien, Gallensäurenverlustsyndrom, Medikamentenanamnese, Infektionen) (IV, starker Konsens, C).

Kommentar: In diesen Situationen kann eine erweiterte Labordiagnostik Aufschlüsse zur Ätiologie der Gallensteine liefern. Insbesondere sind hereditäre Formen der Cholelithiasis auszuschließen [3]. Bei hämolytischer Anämie ist an Hämoglobinelektrophorese (Sichelzellanämie, Thalassämie), osmotische Resistenz der Erythrozyten und Blutausstrich (Sphärozytose, Elliptozytose) sowie Glukose-6-phosphatdehydrogenase- und Pyruvatkinase-Mangel $z u$ denken. Die hämolytischen Anämien prädisponieren ebenso wie die Mukoviszidose zur Bildung „schwarzer“ Pigmentsteine (Kalziumbilirubinatsteine) in der Gallenblase. Auf die Mukoviszidose, bei der die Gallensteinprävalenz auf 15-30\% im Vergleich zu 5\% bei altersentsprechenden Kontrollen erhöht ist [40], können Diarrhöen und Gallensäurenverlustsyndrom hinweisen.

Generell beträgt die erbliche Komponente bei der Entstehung von Gallenblasensteinen etwa 25\% [41 - 44]. Bei symptomatischer Cholelithiasis vor dem 40. Lebensjahr, Auftreten von biliären Schmerzen nach Cholezystektomie oder sonografischem Verdacht auf intrahepatische Mikrolithiasis bzw. Sludge kann eine mit einer ABCB4-Defizienz assoziierte Cholelithiasis vorliegen [45-47]. Diagnostisch können eine Lipidanalyse von Leber- oder Duodenalgalle und genetische Tests auf Mutationen des hepatobiliären Phospholipidtransporters $A B C B 4$ in Forschungslaboren durchgeführt werden. Bei ABCB4-Defizienz ist die Phospholipidkonzentration im Verhältnis zu den Gallensäuren- und den Cholesterinkonzentrationen in der Galle vermindert [45]. Genetische Studien konnten kürzlich nachweisen, dass eine häufige Variante des ABCG8-Gens des hepatobiliären Cholesterintransporters das Risiko, Cholesteringallensteine zu entwickeln, mehr als verdoppelt [48, 49]. Seltene Mutationen im ABCB11-Gen des Gallensäurentransporters können infolge niedriger Gallensäurenkonzentrationen ebenfalls zur Cholesterinsteinbildung und zur rekurrierenden intrahepatischen Cholestase führen [50]. 


\section{Chirurgische Therapie bei Gallenblasensteinen}

5. Die symptomatische Cholezystolithiasis ist in der Regel eine Indikation zur Cholezystektomie (Ib, starker Konsens, A).

Kommentar: Die Cholezystektomie bei symptomatischer Cholezystolithiasis sollte erfolgen, da abhängig von Intensität und Anzahl der symptomatischen Episoden etwa die Hälfte der Patienten neuerliche Koliken bekommt [51] und das Risiko, Komplikationen zu entwickeln, 1 -3\% pro Jahr beträgt [34, 36, 52, 53]. Die randomisierte National Cooperative Gallstone Study [33] bot die Möglichkeit, den natürlichen Spontanverlauf bei Patienten zu beobachten, die keine Litholyse mit Chenodeoxycholsäure erhielten. Von diesen Patienten entwickelten 69\% innerhalb von zwei Jahren erneut biliäre Symptome, und 4\% der Patienten mussten akut cholezystektomiert werden [33].

Während die Cholezystektomie die Komplikationen der Gallenblasensteine verhindert, lässt sich das Komplikationsrisiko von in den Gallengang übergetretenen Steinen, insbesondere das der biliären Pankreatitis, auch durch die endoskopische Papillotomie (EPT) verringern; falls die Gallenblase in dieser Situation funktionstüchtig und steinfrei ist, muss nicht cholezystektomiert werden (siehe III.11). Liegt die letzte Gallenkolik länger als 5 Jahre zurück, ist eine Cholezystektomie in aller Regel ebenfalls nicht erforderlich (erneut asymptomatischer Steinträger) [34]. Nach einer Cholezystektomie können abdominelle Beschwerden mit meist unspezifischem Charakter bei jedem dritten bis vierten Patienten persistieren [32, 54-58].

6. Die asymptomatische Cholezystolithiasis ist in der Regel keine Indikation zur Therapie (III, starker Konsens, B).

Kommentar: Diese Empfehlung wurde von der Konsensuskonferenz in Übereinstimmung mit den Patientenvertretern in die Kategorie $B$ eingestuft, da weder umfangreiche klinische Beobachtungen noch detaillierte Analysen prospektiver Studien zum klinischen Verlauf der asymptomatischen Cholezystolithiasis den Nutzen der Cholezystektomie bei asymptomatischen Steinträgern belegen. Zum natürlichen Verlauf der asymptomatischen Gallensteinerkrankung gibt es sieben Studien, in denen insgesamt 823 Patienten über einen Zeitraum von 2-25 Jahren beobachtet wurden. In diesem Zeitraum blieben $60-80 \%$ der Patienten asymptomatisch. Die Wahrscheinlichkeit, biliäre Symptome zu entwickeln, beträgt in den ersten fünf Jahren nach Diagnosestellung 2-4\% pro Jahr und halbiert sich in den folgenden Jahren auf 1-2\% [34, 36, 52, 59, 60]. Die jährliche Inzidenz von Komplikationen liegt bei 0,1 - 0,3\% [34, 36]. Die Behandlung (offene Cholezystektomie) des asymptomatischen Gallenblasensteinträgers erhöht dessen Lebenserwartung nicht, da das Operationsrisiko (Letalität und Morbidität) die Wahrscheinlichkeit, Komplikationen zu erleiden, aufwiegt [35, 61]. Ferner sind die Kosten geringer, wenn man sich bei asymptomatischen Gallensteinträgern bis zum Auftreten von Symptomen oder Komplikationen abwartend verhält und weder eine prophylaktische Cholezystektomie noch eine Litholyse (siehe II.13) durchführt [62]. In Ländern mit niedriger Gallenblasenkarzinomprävalenz [63] rechtfertigt das leicht erhöhte, aber immer noch sehr niedrige Gallenblasenkarzinom-Risiko bei der asymptomatischen Cholezystolithiasis nicht den Eingriff [64, 65]. Auch Diabetiker bedürfen keiner prophylaktischen Therapie [66, 67]. Da sich die bisherigen Kosten-Nutzen-Analysen bei asymptomatischen bzw. symptomarmen Patienten auf die offene Cholezystektomie bezogen, sind weitere Studien sinnvoll, die das höhere Lebensalter der Bevölkerung, die Lebensqualität der Patienten und die laparoskopische Cholezystektomie als aktuellen Standardeingriff berücksichtigen.

\section{Ausnahmen:}

a) Asymptomatische Patienten mit Porzellangallenblase sollten cholezystektomiert werden (III, starker Konsens, B).

Kommentar: Diese Empfehlung wurde von den Patientenvertretern und den anderen Teilnehmern der Konsensuskonferenz aufgrund der Hinweise, dass ein relativ hoher Prozentsatz dieser Patienten ohne rechtzeitige Cholezystektomie ein Gallenblasenkarzinom entwickelt, in die Kategorie B eingestuft. Nach früheren Arbeiten findet sich in bis zu 20\% aller kalzifizierten Gallenblasen ein Karzinom [68], obgleich dieser Zusammenhang nicht in allen Serien bestätigt wurde [69]. Es muss allerdings zwischen homogenen Wandverkalkungen (Karzinomrate sehr niedrig) und fleckförmigen Verkalkungen (Karzinomrate 7\%) unterschieden werden [70]. Bei Patienten mit homogenen Wandverkalkungen kann ggf. auf die Cholezystektomie verzichtet werden [67]. Die Porzellangallenblase kann sonografisch mit hoher Zuverlässigkeit diagnostiziert werden. Eine Bestätigung durch CT ist präoperativ empfehlenswert.

b) Bei asymptomatischen Patienten mit Gallenblasensteinen $>\mathbf{~ c m}$ Durchmesser ist eine Cholezystektomie vertretbar (IIb, starker Konsens, B).

Kommentar: Bei männlichen Steinträgern mit Konkrementen $>3 \mathrm{~cm}$ ist das Risiko, ein Gallenblasenkarzinom zu erleiden, 9- bis 10-fach erhöht [71, 72]. Obwohl Gallensteine mit einem erhöhten Risiko für ein Gallenblasenkarzinom einhergehen, sind sie generell keine Indikation zur prophylaktischen Cholezystektomie (siehe II.6). Je nach zusätzlichen Risikofaktoren müssen zwischen 67 und 769 Cholezystektomien durchgeführt werden, um ein Gallenblasenkarzinom zu verhindern [73].

c) Patienten mit Cholezystolithiasis und Gallenblasenpolypen $\geq 1 \mathrm{~cm}$ sollten unabhängig von der Symptomatik cholezystektomiert werden (IIb, starker Konsens, B).

Kommentar: Die Prävalenz von Gallenblasenpolypen liegt zwischen 1\% und 7\% [74-77], in Deutschland beträgt sie 1,5\% [78]. Die Adenom-Prävalenz liegt unter 5\% [76, 79]. In mehreren großen Studien [76, 77, 80-83] hatten Polypen $\geq 1 \mathrm{~cm}$ Durchmesser eine deutlich erhöhte Wahrscheinlichkeit, neoplastischer Genese (Adenome) zu sein, und in bis zu 50\% das Risiko, ein Karzinom zu tragen [76, 77, 80-83], so dass diese Patienten cholezystektomiert werden sollten. Bei Polypen $<1 \mathrm{~cm}$ Durchmesser war das Karzinomrisiko deutlich geringer [84, 85]. Falls die Patienten jedoch gleichzeitig biliäre Symptome oder weitere Risikofaktoren für eine Adenomentwicklung (Alter > 50 Jahre, solitäre Polypen, Gallensteine) haben, kann auch in diesen Fällen eine Cholezystektomie durchgeführt werden $[76,82,86]$.

Bei Polypen > 18-20 mm sollte wegen des signifkanten Malignitätsrisikos primär die offene Cholezystektomie erwogen werden $[79,83,86,87]$. Bei Polypen $<1 \mathrm{~cm}$, die nicht operiert werden, sollten sonografische Verlaufskontrollen vorgenommen werden (zunächst alle 6 Monate, später jährlich, wenn keine Größenzunahme vorliegt) [77, 83]. Während der Sonografie sind Gallenblasenpolypen durch Umlagern des Patienten von Gallenblasensteinen zu differenzieren. Das Vorliegen von mehr als einem Polypen spricht gegen ein Adenom und für das Vorliegen von Cholesterolpolypen. Farbduplexsonografisch gelingt mitunter der für Adenome typische Nachweis von Gefäßen in der Polypenbasis [88]. Gallenblasenpolypen können mit der Endosonografie präziser als mit der transkutanen Sonografie erfasst werden (87-97\% versus 52 - 76\%) [89, 90]. Bei Gallenblasenpolypen > $5 \mathrm{~mm}$, die in der transkutanen Sonografie suspekt sind, kann die Endosonografie daher zur Abgrenzung von einem Gallenblasenkarzinom hilfreich sein. 
Die Situation von polypoiden Gallenblasenveränderungen ohne gleichzeitige Cholezystolithiasis wurde im Konsensus nicht berücksichtigt.

d) Bei großen abdominellen Eingriffen (z. B. malabsorptive/restriktive Adipositaschirurgie, ausgedehnte Resektion bei Morbus Crohn, radikale Gastrektomie mit Lymphadenektomie) kann eine simultane Cholezystektomie auch bei asymptomatischen Steinen vorgenommen werden (III, starker Konsens, C).

Kommentar: Das Risiko für Gallenblasensteinträger, nach malabsorptiver/restriktiver Adipositaschirurgie symptomatisch zu werden bzw. Komplikationen zu entwickeln, beträgt 10 bis 15\% [91, 92], so dass die prophylaktische Cholezystektomie zu erwägen ist. Obgleich bei M. Crohn, insbesondere nach mehrfachen Darmresektionen [93, 94], und nach radikaler Gastrektomie mit Lymphadenektomie $[95,96]$ das Steinrisiko wahrscheinlich erhöht ist, wird in dieser Situation bei steinfreier Gallenblase die Cholezystektomie nicht generell empfohlen [97]. Das Steinrisiko steigt insbesondere nach Ileozökalresektion, bei Kurzdarmsyndrom mit weniger als $120 \mathrm{~cm}$ und bei längerfristiger parenteraler Ernährung an [98].

Während die Nierentransplantation [99-101] und wahrscheinlich auch die kombinierte Pankreas-/Nieren-Transplantation [102] keine Indikation zur prophylaktischen Cholezystektomie darstellen, senkt diese bei asymptomatischen Gallenblasensteinträgern nach der Herztransplantation die Letalität und ist kosteneffektiv $[102,103]$

7. Die laparoskopische Cholezystektomie ist die Standardtherapie für die symptomatische Cholezystolithiasis; dies schließt die akute Cholezystitis und in der Regel auch Gallenblasensteine mit anderen Komplikationen ein (la, starker Konsens, A).

Kommentar: Die laparoskopische Cholezystektomie ist weltweit zum Standardeingriff geworden. Mehr als 93\% aller Cholezystektomien werden heute laparoskopisch begonnen, die Konversionsrate auf die offene Cholezystektomie beträgt 4 - 7\% [104-107]. Die aktuelle Metaanalyse [108] randomisierter kontrollierter Studien, die beide Verfahren verglichen [109-143], zeigt für die laparoskopische Cholezystektomie identische Komplikationsraten bei einer im Mittel um 3 Tage kürzeren Krankenhaus-Verweildauer und einer um 3 Wochen kürzeren Rekonvaleszenz. Dies spiegelt sich in Kostenanalysen wider, die für die stationäre Behandlung beim laparoskopischen Verfahren um 18\% niedrigere Kosten als bei offener Cholezystektomie ermittelten [144]. Auch im historischen Vergleich sind die aktuellen Komplikationsraten (Gallenleck 0,4-1,5\%, Wundinfektion 1,3-1,8\%, Pankreatitis 0,3\%, Blutung 0,2-1,4\%) geringer als bei der offenen Cholezystektomie [5, 104, 107]. Nachdem eine große Metaanalyse 1996 [145] noch einen Trend zu mehr Gallengangsverletzungen vermutete, ist die Gallengangsverletzungsrate nach Cholezystektomie heute verfahrensunabhängig niedrig $(0,2-0,4 \%)[107,108,146,147]$. Eine Überlegenheit eines der Verfahren kann in diesem Zusammenhang wegen mangelnder Vergleichbarkeit der Kollektive weder aus der gegenwärtigen Literatur noch aus den aktuellen Daten der Erhebung der Bundesgeschäftsstelle Qualitätssicherung (BQS) abgeleitet werden. In der BQS-Erhebung 2005 wurden bei 0,15\% der laparoskopisch begonnenen Cholezystektomien eine Okklusion oder Durchtrennung des Ductus hepatocholedochus gemeldet [147].

Randomisierte kontrollierte Studien zeigen, dass auch die akute Cholezystitis laparoskopisch operiert werden kann [148-150], wobei Operationszeit, Risiken und Konversionsraten höher liegen als bei der elektiven Cholezystektomie. In der deutschen Studie [148] führte die laparoskopische Cholezystektomie im Vergleich zur offenen Cholezystektomie zu einer Verkürzung der Krankenhaus-Verweildauer von 8,5 auf 5,8 Tage und zu einer Abnahme der Gesamt-Komplikationsrate von $22,4 \%$ auf $12,4 \%$.

Bei manifester portaler Hypertension und bei Leberzirrhose im Child-Stadium A/B ist die laparoskopische Cholezystektomie einer offenen Operation möglicherweise überlegen [151], wohingegen bei Child-C-Patienten [152] - möglicherweise aber auch bereits bei Patienten mit einem MELD-Score $\geq 8$ [153] - unabhängig vom Verfahren das Komplikationsrisiko der Cholezystektomie hoch ist. Auf die laparoskopische Cholezystektomie sollte primär zugunsten der offenen Cholezystektomie beim dringenden Verdacht auf ein (fortgeschrittenes) Gallenblasenkarzinom verzichtet werden. Das Vorliegen eines Mirizzi-Syndroms - sofern es präoperativ erkannt wurde - ist nicht per se eine Kontraindikation gegen das laparoskopische Vorgehen; allerdings sollte gerade bei Mirizzi II (Fistel zwischen Gallenblase und Ductus hepaticus) eine hohe Bereitschaft zur Konversion bestehen [154, 155].

Die randomisierten kontrollierten Studien, welche die laparoskopische Cholezystektomie mit der Mini-Laparotomie-Cholezystektomie (Laparotomie $<8 \mathrm{~cm}$ ) verglichen, fanden keine Unterschiede zwischen beiden Verfahren hinsichtlich Komplikationsraten, Krankenhaus-Verweildauern und Rekonvaleszenzzeiten [111, 156-167]. Ein weiteres neues Verfahren, die minilaparoskopische Cholezystektomie, führte in randomisierten kontrollierten Studien in der Hand erfahrener laparoskopischer Chirurgen bei tendenziell längeren OP-Zeiten etwas seltener zu postoperativen Schmerzen als die laparoskopische Cholezystektomie [168-173].

\section{Therapie bei akuter Cholezystitis}

8. Die akute Cholezystitis ist eine Indikation zur frühelektiven laparoskopischen Cholezystektomie (möglichst innerhalb von $72 \mathrm{~h}$ nach Diagnosestellung) (la, starker Konsens, A).

Kommentar: Die akute Cholezystitis ist die häufigste Komplikation des Gallensteinleidens. Bei 90\% der Patienten mit akuter Cholezystitis ist die Ursache ein passagerer oder dauerhafter Verschluss des Ductus cysticus durch einen Gallenstein. Berichte in den 70er-Jahren zeigten bereits, dass in der Regel die frühe Operation innerhalb von 1 - 5 Tagen nach Diagnosestellung gegenüber der Spätoperation (nach 6-8 Wochen) für den Patienten Vorteile hat. Fünf randomisierte kontrollierte Studien belegten eine deutlich kürzere Krankenhaus-Verweildauer bei der frühelektiven Cholezystektomie im Vergleich zur Spätoperation (11 versus 20 Tage), geringere Kosten sowie eine Verringerung der Morbidität und Letalität [174 - 178]. Nach diesen Studien folgten vier weitere randomisierte kontrollierte Studien [179-182], die bei insgesamt 388 Patienten die frühe und die späte Cholezystektomie (> 6 Wochen) für das laparoskopische Vorgehen verglichen ( Tab. 2). Metaanalysen dieser Studien bestätigten die Vorteile der frühelektiven laparoskopischen Cholezystektomie [183]. Die Krankenhaus-Verweildauer ist bei der Spätoperation 3 Tage länger, und es mussten 17,5\% der Patienten während der präoperativen Wartephase notfallmäßig operiert werden [183]. Das frühe operative Vorgehen erhöht nicht die Komplikationsrate (13,1\% versus 17,8\%) im Vergleich zur späten Cholezystektomie [184]. Die Konversionsrate bei der akuten Cholezystitis beträgt 16,4-20,3\% [183, 185] und liegt damit deutlich höher als bei der elektiven Operation nach symptomatischer Cholezystolithiasis (siehe II.7). Bis zur Operation sollte der Patient konservativ durch Flüssigkeitssubstitution, Antibiotikagabe und Elektrolytausgleich behandelt werden. Die Operation sollte bald nach der Feststellung der Narkose- und Operationsfähigkeit erfolgen, da die Konversionsraten eher niedriger und der 
Tab.2 Randomisierte kontrollierte Studien zur früh- versus spätelektiven laparoskopischen Cholezystektomie bei akuter Cholezystitis

\begin{tabular}{|c|c|c|c|c|c|c|c|c|c|}
\hline Studie & Land & Operation & $\mathbf{n}$ & $\begin{array}{l}\text { Letalität } \\
\text { (\%) }\end{array}$ & $\begin{array}{l}\text { Kompli- } \\
\text { kationen } \\
(\%)\end{array}$ & $\begin{array}{l}\text { Operations- } \\
\text { dauer } \\
\text { (min) }\end{array}$ & $\begin{array}{l}\text { Konver- } \\
\text { sionsrate } \\
(\%)\end{array}$ & $\begin{array}{l}\text { Krankenhaus- } \\
\text { Verweildauer } \\
\text { (Tage) }\end{array}$ & $\begin{array}{l}\text { Gallengangs- } \\
\text { verletzungen } \\
(\%)\end{array}$ \\
\hline \multirow[t]{2}{*}{ Lai et al. 1998 [180] } & \multirow[t]{2}{*}{ China } & früh & 53 & 0 & 9,4 & 123 & 20,8 & 7,6 & 0 \\
\hline & & spät & 51 & 0 & 6,5 & 107 & 23,9 & 11,6 & 0 \\
\hline \multirow{2}{*}{ Lo et al. 1998 [179] } & \multirow{2}{*}{ China } & früh & 49 & 0 & 13,3 & 135 & 10,4 & 6,0 & 0 \\
\hline & & spät & 50 & 0 & 29,3 & 105 & 20,0 & 11,0 & 2,2 \\
\hline \multirow{2}{*}{$\begin{array}{l}\text { Johansson et al. } \\
2003 \text { [181] }\end{array}$} & \multirow[t]{2}{*}{ Schweden } & früh & 74 & 0 & 17,6 & 98 & 31,1 & 5,0 & 0 \\
\hline & & spät & 71 & 0 & 10,1 & 100 & 29,0 & 8,0 & 1,5 \\
\hline \multirow{2}{*}{$\begin{array}{l}\text { Kolla et al. } 2004 \\
\text { [182] }\end{array}$} & \multirow[t]{2}{*}{ Indien } & früh & 20 & 0 & 20,0 & 104 & 25,0 & 4,1 & 5,0 \\
\hline & & spät & 20 & 0 & 15,0 & 93 & 25,0 & 10,1 & 0 \\
\hline
\end{tabular}

Krankenhausaufenthalt eher kürzer sind, je früher operiert wird [186].

Die konservative Therapie der akuten Cholezystitis ist zwar möglich, allerdings kommt es nach alleiniger konservativer Behandlung bei über einem Drittel dieser Patienten zu Komplikationen oder Notaufnahmen wegen biliärer Schmerzen, und bei $30 \%$ wird im weiteren Verlauf eine Cholezystektomie erforderlich $[187,188]$. Alternativ kann bei älteren Patienten (> 65 Jahre) oder erhöhtem Operationsrisiko (ASA-Risikoklasse $\geq$ III; $\bullet$ Tab. 5) eine perkutane Drainage der Gallenblase (Cholezystostomie) mit niedrigen Letalitäts- und hohen Erfolgsraten erwogen werden [187, 189, 190]. Kann wegen zu später Diagnosestellung oder aus anderen medizinischen Gründen ( $z$ u hohes OP-Risiko) der Patient nicht frühelektiv innerhalb der ersten 1 -5 Tage operiert werden [191], sollte die Cholezystektomie dann erst nach 6 Wochen im Intervall erfolgen [192].

Bei 10 bis 30\% der Patienten mit akuter Cholezystitis treten schwere Komplikationen wie Gallenblasengangrän, -empyem oder -perforation auf $[149,193,194]$. In diesen Situationen kann ein präoperatives CT hilfreiche Informationen liefern. Fisteln zwischen der Gallenblase und dem Gastrointestinaltrakt entwickeln sich bei weniger als 1\% aller Gallensteinpatienten. Klinisch kann sich eine biliodigestive Fistel durch aszendierende Cholangitis oder Gallensäurenverlustsyndrom manifestieren. In der Regel (60\%) handelt es sich um cholezystoduodenale Fisteln, die oft asymptomatisch sind. Wenn größere Steine durch die Fisteln abgehen, kann es zum Bild des Gallensteinileus kommen, insbesondere im terminalen Ileum [195]. Der Nachweis der Aerobilie ist diagnostisch wegweisend für eine biliodigestive Fistel; zur weiteren Diagnostik sind Magnetresonanztomografie mit Magnetresonanzcholangiografie (MRC) und endoskopische Verfahren geeignet.

\section{Präoperative Diagnostik}

9. Folgende diagnostische Maßnahmen sind vor einer Cholezystektomie notwendig: transkutane B-Mode-Sonografie sowie Bestimmung von Cholestaseenzymen ( $\gamma$-GT, AP), ALT, Bilirubin, Lipase, Gerinnungsparametern (INR, PTT) und kleines Blutbild (IV, starker Konsens, C).

Kommentar: Die präoperative Sonografie dient dem Nachweis bzw. dem Ausschluss der Cholezystolithiasis mit und ohne Komplikationen. Obgleich erfahrene Untersucher unter Einsatz des Farbdopplers darüber hinaus aberrante und pathologische Gefäßverläufe (z.B. Varianten der arteriellen Versorgung, Topografie der mittleren Lebervene, Kollateralkreisläufe) erkennen können [196-200], ist dieses zur präoperativen Risikoeinschätzung hilfreiche Verfahren nicht zwingend erforderlich.

Die präoperative Bestimmung von Cholestaseenzymen, Transaminasen und Bilirubin ist notwendig, um den Verdacht auf Gallen- gangssteine oder eine vorbestehende Leberkrankheit weitgehend auszuschließen bzw. zu bestätigen [201, 202]. Keine Operation sollte ohne Kenntnis der Gerinnungsparameter (INR, PTT) durchgeführt werden. Ein präoperatives Blutbild gehört zum Standard, um bei möglichen Komplikationen (Blutung, postoperative Entzündung) den Verlauf beurteilen zu können.

Bei jüngeren Patienten ohne Begleitkrankheiten kann auf eine präoperative Röntgen-Thoraxaufnahme verzichtet werden [203], bei älteren Patienten mit vorbestehenden Krankheiten des Herzens oder der Lungen sollten jedoch präoperativ eine Thoraxaufnahme und ein EKG durchgeführt werden. Eine Ösophagogastroduodenoskopie ist vor einer geplanten Cholezystektomie nicht generell erforderlich [204, 205]. Sie sollte jedoch dann durchgeführt werden, wenn eine Ulkusanamnese besteht, der Patient nichtsteroidale Antirheumatika (NSAR) einnimmt oder uncharakteristische Beschwerden im Oberbauch bestehen.

Bei Verdacht auf das gleichzeitige Vorhandensein von Gallengangssteinen sind präoperativ weitere diagnostische Maßnahmen (endoskopische retrograde Cholangiografie [ERC], Endosonografie oder MRC) indiziert, deren Einsatz in den Empfehlungen III.3 und III.6 behandelt wird. Eine Routine-ERC vor Cholezystektomie wird nicht empfohlen.

\section{Therapie bei Gallenblasenkarzinom}

10. Wird postoperativ ein Carcinoma in situ (Tis) oder ein Mukosakarzinom (T1a) festgestellt, ist die Entfernung der Gallenblase ausreichend (Ila, starker Konsens, A).

Kommentar: Bei 0,2-0,4\% der Cholezystektomien findet sich ein Gallenblasenkarzinom. Der Anteil der zum Zeitpunkt der Diagnose potenziell resektablen Gallenblasenkarzinome liegt bei $10-30 \%$ mit einer 5-Jahres-Überlebensrate von 10-60\% nach R0-Resektion [206, 207]. Bei einem kleinen Gallenblasenkarzinom (T1a) mit serosanahem Sitz ist eine lymphogene Metastasierung selten ( $<2,5 \%$ ). Es reicht deshalb in der Regel die Cholezystektomie aus. Bezüglich des therapeutischen Vorgehens im Tumorstadium T1b (Infiltration der Muscularis) besteht derzeit kein Konsens [206]. Obgleich es in retrospektiven Analysen [208, 209] und im Zentralregister für okkulte Gallenblasenkarzinome (siehe IV.3) Hinweise gibt, dass eine Nachresektion (siehe II.10) im Stadium T1b einen prognostischen Vorteil besitzt, bietet die Datenlage keine Grundlage, um dieses Vorgehen generell zu empfehlen [210-213].

11. Falls nach Cholezystektomie wegen Cholezystolithiasis postoperativ ein Tumor im Stadium $\geq \mathrm{T} 2$ nachgewiesen wird, ist grundsätzlich eine Reoperation mit kurativem Ansatz indiziert (III, starker Konsens, C). Die Wirksamkeit adjuvanter Therapiemaßnahmen nach R0-Resektion oder einer postoperativen medikamentösen Therapie bzw. Strahlentherapie nach R1- und 
R2-Resektion ist bisher für das Gallenblasenkarzinom nicht bewiesen (IV, starker Konsens, C).

Kommentar: Gallenblasenkarzinome zeigen eine frühe lymphogene Metastasierung. Bei T2/T3-Tumoren (Infiltration des perimuskulären Bindegewebes bzw. Perforation der Serosa) wird daher in der Regel eine radikale Nachresektion, ggf. als Bisegmentektomie (Segmente IVb und V), angeschlossen [214, 215]. Dadurch ließ sich bei T2-Tumoren in japanischen Studien das 5-Jahresüberleben von $19-40 \%$ auf $61-90 \%$ anheben [206, 215-218]. Es sollte eine Lymphadenektomie, die das Ligamentum hepatoduodenale bis zum Truncus coeliacus umfasst und primär prognostischen Wert besitzt, angeschlossen werden [219]. Nach einer laparoskopischen Cholezystektomie mit postoperativer Karzinomdiagnose ist zu beachten, dass die Gefahr von Impfmetastasen besteht, weshalb empfohlen wird, die Trokareinstichkanäle zu exzidieren [220-222]. Obgleich in einer randomisierten kontrollierten Studie aus Japan eine Chemotherapie mit Mitomycin C und 5-Fluorouracil das 5-Jahres-Überleben bei Patienten nach Resektion eines Gallenblasenkarzinoms verlängerte [223], fehlen größere prospektive Studien, um den Wert der adjuvanten Therapie beurteilen zu können [206, 224]. Patienten mit fortgeschrittenen Gallenblasenkarzinomen sollten daher in klinische Studien eingebracht werden.

\section{Therapie in der Schwangerschaft}

12. Im ersten und zweiten Trimenon einer Schwangerschaft kann eine laparoskopische Cholezystektomie bei dringlicher Indikation durchgeführt werden (III, starker Konsens, C). Patientinnen mit gleichzeitig vorliegenden Gallenblasen- und Gallengangssteinen, die nach der Gallengangssanierung asymptomatisch sind, sollten erst post partum cholezystektomiert werden (IV, starker Konsens, C).

Kommentar: Gallenblasen-Sludge oder Gallensteine bilden sich bei je 5\% der Schwangeren aus, und 1,2\% der Schwangeren entwickeln biliäre Schmerzen [225]. Asymptomatische Steinträgerinnen werden nicht behandelt (siehe II.5). Allerdings wird bei diesen Patientinnen häufig im ersten Jahr nach der Schwangerschaft eine Cholezystektomie nötig [226].

Die Schwangerschaft ist grundsätzlich keine Kontraindikation zur Cholezystektomie [227-229]. Ein konservatives Vorgehen bei symptomatischen Patientinnen führte in einer retrospektiven Analyse bei bis zu 40\% der Patientinnen noch während der Schwangerschaft zum Rezidiv der Beschwerden [230]. Die aktuelle Datenlage und Erfahrungswerte zeigen, dass auch im ersten Trimenon bei dringlicher Indikation eine laparoskopische Cholezystektomie sicher durchgeführt werden kann [231 -233]. Der intraabdominelle Druck sollte hierbei unter $12-14 \mathrm{mmHg}$ liegen, und es sollte intraoperativ ein fetales Monitoring erfolgen [234, 235]. Im dritten Trimenon sollte die Indikation wegen der Gefahr der Auslösung vorzeitiger Wehen und der beengten Verhältnisse sehr streng gestellt werden. Für alle in der Literatur berichteten laparoskopischen Cholezystektomien lag die fetale Sterblichkeit bei 4\%; bei der offenen Cholezystektomie betrug sie 5\% [235].

\section{Konservative Therapie der Gallenblasensteine}

13. Die Litholyse mit Ursodeoxycholsäure (UDCA) kann in Einzelfällen bei symptomatischen Gallenblasensteinen ohne Komplikationen mit leichten und/oder seltenen Koliken durchgeführt werden; zuvor muss der Patient insbesondere auf das hohe Rezidivsteinrisiko aufmerksam gemacht werden (la, starker Konsens, A).

Kommentar: Die Metaanalyse von Studien zur Litholyse mit UDCA [236] ergab akzeptable Therapieerfolge (Steinfreiheit bis zu
60\% nach 6 Monaten) vor allem bei Patienten mit einer funktionstüchtigen Gallenblase und multiplen röntgennegativen Cholesterinsteinen $<$ 5-10 $\mathrm{mm}$ [236], wobei die konservative Therapie heute wegen der besseren Ergebnisse nur bei Konkrementen $<5 \mathrm{~mm}$ erwogen werden sollte (siehe II.14). Für die Litholyse kommen insbesondere Patienten mit hohem Operationsrisiko infrage. Heute wird zur Litholyse die Monotherapie mit UDCA empfohlen, da sie bei vergleichbarer Effektivität [237] nicht mit den möglichen Nebenwirkungen der Chenodeoxycholsäure (Anstieg der Transaminasen durch Lebertoxizität, leichter Anstieg des LDL-Cholesterins im Serum) belastet ist. Auch treten Durchfälle deutlich seltener unter Monotherapie mit UDCA als unter der Kombination von UDCA und Chenodeoxycholsäure auf.

Möglicherweise kann eine niedrig dosierte UDCA-Therapie ( $2 \times 150 \mathrm{mg} / \mathrm{Tag})$ die Inzidenz dyspeptischer Beschwerden bei Patienten mit Gallensteinen reduzieren [238, 239]. Die zunächst in einer japanischen Kohortenanalyse aufgefallene, von der Litholyse unabhängige Verminderung des Risikos biliärer Schmerzen oder einer akuten Cholezystitis [240] konnte in einer aktuellen Studie in den Niederlanden nicht bestätigt werden; hier hatte UDCA bei Patienten auf der Warteliste zur Cholezystektomie keinen Nutzen [51].

14. Für die Litholyse mit UDCA eignen sich Patienten mit kleinen ( $\leq 5 \mathrm{~mm}$ ), nicht kalkhaltigen Steinen in einer sonografischen Untersuchung gut kontrahierende Gallenblase (Ib, starker Konsens, A). Als Dosis werden mindestens 10 mg UDCA/kg/Tag empfohlen (lb, starker Konsens, A). Die Therapie sollte nach sonografisch verifizierter Steinfreiheit noch für 3 Monate fortgesetzt werden (IV, starker Konsens, C).

Kommentar: Eine funktionstüchtige Gallenblase mit durchgängigem Ductus cysticus ist die Voraussetzung für die medikamentöse Litholyse [241]. Dies kann durch eine Sonografie mit Reizmahlzeit (Ejektionsfraktion $\geq 60 \%$ ) geprüft werden [242]. Bei Verkalkungen und mit zunehmender Dichte der Steine (CT, Gallenblasenzielaufnahme) nehmen die Erfolgsraten der Litholyse ab; das CT ist zum Nachweis von Verkalkungen am sensitivsten [244].

Laborchemisch sollten vor der Litholyse mit UDCA Cholestaseenzyme ( $\gamma$-GT, AP), ALT und ein kleines Blutbild bestimmt werden. Die UDCADosis sollte mindestens $10 \mathrm{mg} / \mathrm{kg}$ pro Tag betragen, am besten als abendliche Einzeldosis [245]. Das Therapieansprechen sollte sonografisch überprüft werden. Obgleich bei größeren Steinen (6-10 mm) die Erfolgsrate deutlich geringer ist und nur 40\% dieser Patienten steinfrei werden [246], kann auch bei diesen größeren Steinen der Versuch einer konservativen Therapie vertretbar sein.

15. Bei neuerlichen Symptomen nach erfolgreicher Therapie muss eine Sonografie durchgeführt werden (IV, starker Konsens, C). Bei symptomatischem Steinrezidiv ist in der Regel die Cholezystektomie indiziert (IV, starker Konsens, C). Bei asymptomatischen Patienten ist der Nutzen von Kontrolluntersuchungen nicht belegt (IV, starker Konsens, C).

Kommentar: Ein Steinrezidiv kann mit sehr hoher Spezifität und Sensitivität sonografisch diagnostiziert werden. Da diese Patienten offensichtlich eine hohe Prädisposition zur Steinentstehung besitzen [247], wird bei ihnen keine erneute konservative Therapie empfohlen. Beim symptomatischen Steinrezidiv sollte daher zur Cholezystektomie geraten werden. Bei asymptomatischen Rezidivsteinpatienten ist der Wert einer prophylaktischen konservativen oder operativen Therapie nicht belegt. Aus diesem Grund sind auch keine regelmäßigen sonografischen Kontrollen nach erfolgreicher konservativer Therapie erforderlich. 
16. Aufgrund der schlechteren Langzeitergebnisse hat sich die extrakorporale Stoßwellenlithotripsie (ESWL) der Gallenblasensteine im Vergleich zur laparoskopischen Cholezystektomie nicht bewährt (Ila, starker Konsens, A).

Kommentar: Mehrere Studien belegen die hohe Rezidivrate im Langzeitverlauf (25-64\% nach 5 Jahren und 54-80\% nach 10 Jahren) [247-257]. Gute Gallenblasenkontraktilität, BMI $<25 \mathrm{~kg} / \mathrm{m}^{2}$ und UDCA-Prophylaxe verzögern die Rezidivsteinbildung, verhindern sie jedoch nicht; Patienten mit Solitärsteinen bekommen seltener Rezidive [247, 254-261]. Die Mehrzahl der Rezidivsteine ist symptomatisch und ein Drittel der Patienten muss im Mittel nach 3 Jahren cholezystektomiert werden [262]. Auch hinsichtlich der Kosten ist die ESWL der laparoskopischen Cholezystektomie unterlegen [263, 264].

17. Die Litholyse mit Methyl-Ter-Butyl-Ether (MTBE) zur Behandlung von Gallenblasensteinen ist obsolet (IV, starker Konsens, C). Kommentar: Bei der Litholyse mit MTBE kann eine weitgehende Steinfreiheit meistens innerhalb einiger Tage erzielt werden, doch ist die Rezidivrate bei dieser Therapieform sehr hoch (70\% innerhalb von 3 Jahren). Auch bietet dieses sehr nebenwirkungsreiche Verfahren hinsichtlich der Krankenhaus-Verweildauer gegenüber der laparoskopischen Cholezystektomie keinen Vorteil [265-267].

\section{Prävention von Gallenblasensteinen}

18. In Situationen, die infolge Gewichtsreduktion mit hohem Risiko zur Bildung von Gallenblasensteinen einhergehen (z. B. Reduktionsdiät, Adipositaschirurgie), kann das Steinrisiko durch UDCA vermindert werden (Ib, starker Konsens, A). Die Dosis sollte mindestens $500 \mathrm{mg} /$ Tag betragen, und die Therapie sollte bis zur Gewichtsstabilisierung fortgeführt werden (Ib, starker Konsens, A).

Kommentar: Adipositas per se und insbesondere abdominelle Adipositas prädisponieren zur Gallensteinbildung und erhöhen das Risiko für eine Cholezystektomie wegen symptomatischer Gallensteine [4, 268, 269], während körperliche Aktivität steinprotektiv ist [270-273]. Bei rascher Gewichtsreduktion (> 1,5 kg/Woche) unter Reduktionsdiät steigt das Steinrisiko signifikant an [274-277]; Gewichtsschwankungen („Weight cycling“) sind ein unabhängiger Risikofaktor [270, 278, 279].

Mehrere randomisierte kontrollierte Studien [274, 280-285] konnten zeigen, dass das Steinrisiko unter Reduktionsdiät mit ra- scher Gewichtsabnahme, nach Magenbypass oder nach Anlage eines Magenbandes durch die Einnahme von UDCA verringert wird ( Tab. 3). Nach Studienlage wird mit einer Dosis, die niedriger als bei der Litholyse mit UDCA liegt, maximal 6 Monate behandelt. Eine Entscheidungsbaumanalyse zeigt, dass die medikamentöse Prävention mit UDCA die Kosten senkt [286].

\section{Therapie der biliären Kolik}

19. Die Therapie der biliären Kolik erfolgt mit Spasmolytika (z. B. $\mathrm{N}$-Butylscopolamin i. v.) in Kombination mit peripher wirksamen Analgetika (z. B. Diclofenac [lb, starker Konsens, A], Indometacin [Ila, starker Konsens, A], Metamizol [Ila, starker Konsens, A], Paracetamol [IV, starker Konsens, C]); bei schwerer Symptomatik können Opiatderivate (z. B. Pethidin, Buprenorphin i. v.) indiziert sein (IV, starker Konsens, C).

Kommentar: Bei der Behandlung der akuten Gallenkolik muss zwischen der unmittelbar erforderlichen medikamentösen Schmerztherapie und der kausalen Therapie (siehe II.5) unterschieden werden. Zur analgetischen Therapie der biliären Kolik werden Spasmolytika in Kombination mit Analgetika eingesetzt. Schwächer wirksame Analgetika, z.B. Metamizol [287] oder Paracetamol, können ausreichen, oder aber es werden stärker analgetisch wirksame Opiatderivate benötigt. Am besten eignen sich Pethidin und Buprenorphin, da sie den Sphincter Oddi weniger kontrahieren als Morphin [288 - 290]. Außer mit den angegebenen Medikamenten sind Gallenkoliken aber auch mit Nitroglyzerin, erfolgreich behandelt worden [291].

Es ist gezeigt worden, dass NSAR wie Diclofenac (z.B. $75 \mathrm{mg}$ i.m.) [292, 293] oder Indometacin (z.B. 50 mg i.v. oder $2 \times 75$ mg Supp.) [294, 295] bei der biliären Kolik eine gute analgetische Wirksamkeit haben. Neuere randomisierte kontrollierte Studien zeigen, dass durch die Gabe von NSAR in der o.a. Dosis auch die Wahrscheinlichkeit, im Verlauf einer biliären Kolik eine akute Cholezystitis zu entwickeln, gesenkt werden kann [293, 296]. Ob die „traditionelle“ Behandlung der biliären Kolik oder die Therapie mit NSAR zu einer effizienteren Schmerzlinderung führen, kann gegenwärtig aufgrund fehlender vergleichender Studien nicht entschieden werden. Die Wertigkeit verschiedener Medikamentenkombinationen wurde bisher ebenfalls nicht ausreichend untersucht.

Tab.3 Randomisierte kontrollierte Studien zur Steinprophylaxe mit Ursodeoxycholsäure bei Gewichtsreduktion

\begin{tabular}{|c|c|c|c|c|c|c|c|c|}
\hline \multirow[t]{2}{*}{ Studie } & \multirow[t]{2}{*}{ Intervention } & \multirow{2}{*}{$\begin{array}{l}\text { Mittleres } \\
\text { Gewicht } \\
\text { (kg) }\end{array}$} & \multirow{2}{*}{$\begin{array}{l}\text { UDCA } \\
\text { (mg/d) }\end{array}$} & \multirow{2}{*}{$\begin{array}{l}\text { Dauer } \\
\text { Monate }\end{array}$} & \multicolumn{2}{|l|}{ UDCA } & \multicolumn{2}{|l|}{ Plazebo } \\
\hline & & & & & Steine & Steine (\%) & Steine & Steine (\%) \\
\hline $\begin{array}{l}\text { Broomfield et al. } 1988 \\
\text { [274] }\end{array}$ & $520 \mathrm{kcal} / \mathrm{d}$ & 106 & 1200 & 4 & $0 / 18$ & 0 & $5 / 19$ & 26 \\
\hline Williams et al. 1993 [280] & Magenbanding & & $10(\mathrm{mg} / \mathrm{kg})$ & & $0 / 27$ & 0 & $8 / 34$ & 24 \\
\hline Worobetz et al. 1993 [281] & Magenbanding & 143 & 1000 & 3 & $0 / 10$ & 0 & $6 / 14$ & 43 \\
\hline Sugerman et al. 1995 [283] & Magenbypass & 140 & $\begin{array}{r}300 \\
600 \\
1200\end{array}$ & 6 & $\begin{array}{l}7 / 53 \\
1 / 61 \\
4 / 63\end{array}$ & $\begin{array}{r}13 \\
2 \\
6\end{array}$ & $18 / 56$ & 32 \\
\hline Shiffman et al. 1995 [282] & $520 \mathrm{kcal} / \mathrm{d}$ & 128 & $\begin{array}{r}300 \\
600 \\
1200\end{array}$ & 3 & $\begin{array}{r}15 / 185 \\
7 / 201 \\
4 / 200\end{array}$ & $\begin{array}{l}8 \\
3 \\
2\end{array}$ & $57 / 202$ & 28 \\
\hline Wudel et al. 2002 [284] & Magenbypass & 159 & 600 & 6 & $7 / 15$ & 47 & $7 / 11$ & 64 \\
\hline Miller et al. 2003 [285] & Magenbanding & 136 & 500 & 6 & $5 / 64$ & 8 & $18 / 60$ & 30 \\
\hline
\end{tabular}




\section{Gallengangssteine}

$\nabla$

Diagnostik der Gallengangssteine

1. Der anamnestisch-klinisch initiierte Nachweis oder Ausschluss einer Choledocholithiasis erfolgt transkutan sonografisch mit mäßiger diagnostischer Genauigkeit (Ila, Konsens, B).

Kommentar: Die Choledocholithiasis ist mittels des transkutanen Ultraschalls schwieriger als die Cholezystolithiasis nachzuweisen, so dass in Studien wechselnde Sensitivitäten zwischen 38\% und $82 \%$ berichtet wurden [297-303]. Im klinischen Alltag gelingt häufig keine komplette Darstellung der extrahepatischen Gallengänge bis zur Papille. Ein sonografisch erweiterter Ductus choledochus ist ein indirektes diagnostisches Kriterium bei Choledocholithiasis (zu Normwerten siehe III.6 und $\bullet$ Tab. 4) [304, 305]. Es sollte eine standardisierte sonografische Untersuchung durch erfahrene Untersucher unter Einsatz der aktuellen hochauflösenden Gerätetechnik erfolgen [303].

Tab.4 Kriterien für eine simultane Choledocholithiasis bei Cholezystolithiasis

hohe Wahrscheinlichkeit einer simultanen Choledocholithiasis
- sonografisch erweiterter Gallengang (> 7 - $10 \mathrm{~mm}$ ) + Hyperbiliru-
binämie + erhöhte $\gamma$-GT/ALT
- Gallengang > $10 \mathrm{~mm}+$ Gallenblasensteine + Koliken
- sonografischer Verdacht auf Stein im Gallengang
mäßige Wahrscheinlichkeit einer simultanen Choledocholithiasis
- keine hohe oder niedrige Wahrscheinlichkeit
niedrige Wahrscheinlichkeit einer simultanen Choledocholithiasis
- Gallengang normal weit
- Cholestaseparameter normwertig

2. Bei Verdacht auf Choledocholithiasis ist die Bestimmung von Bilirubin, Cholestaseenzymen ( $\gamma$-GT, AP), ALT, Bilirubin und Lipase unerlässlich (la, Konsens, A). Vor endoskopischen Eingriffen ist die Bestimmung von Gerinnungsparametern (INR, PTT) und kleines Blutbild notwendig (IV, Konsens, C).

Kommentar: In Metaanalysen erwiesen sich vor allem das klinische Bild der Cholangitis, der im Ultraschall vermutete Stein, Ikterus, Hyperbilirubinämie und ein sonografisch dilatierter Gallengang als starke Hinweise auf eine Choledocholithiasis [201, 306]. Weitere Studien zeigten darüber hinaus auch den prädiktiven Stellenwert einer Erhöhung der Transaminasen und der Cholestaseenzyme (AP, $\gamma$-GT) [307-320]. Vorhersagemodelle für Gallengangssteine, die auf diesen Parametern basieren, können eine hohe Genauigkeit erreichen, wurden aber nur in wenigen Fällen in einer unabhängigen Kohorte validiert [202] (siehe III.6 und - Tab. 4). Wenn keiner der aufgeführten klinischen oder biochemischen Indikatoren vorliegt, hat dies den gleichen negativen prädiktiven Wert wie eine ERC ohne Steinnachweis [321]. Bei erhöhter Serumlipase ist an eine biliäre Pankreatitis zu denken (siehe III.13).

3. Wenn der direkte sonografische Nachweis oder Ausschluss von Gallengangssteinen nicht gelingt, bestimmen die klinischen Symptome und die Zeichen der biliären Abflussbehinderung den Einsatz der weiteren diagnostischen Maßnahmen, der sich auch nach ihrer Verfügbarkeit richtet (IIb, Konsens, B).

Kommentar: Bei hochgradigem Verdacht auf Gallengangssteine ist die ERC indiziert, da sie auch die gleichzeitige therapeutische Intervention erlaubt (EPT und Steinextraktion). Die ERC hat für die Choledocholithiasis eine Sensitivität und Spezifität > 90\% [299, 300, 322 - 325]. Da die Prävalenz von Gallengangssteinen bei Patienten mit Cholezystolithiasis altersabhängig zwischen 5 und 15\% liegt [326, 327], setzte eine generelle Empfehlung zum Ausschluss von Gallengangssteinen vor einer Cholezystektomie mittels ERC zu viele Patienten unnötig den Risiken dieses diagnostischen Verfahrens aus. Cholangitis, Ikterus und sonografischer Nachweis von Gallengangssteinen sind die besten prädiktiven Hinweise auf das Vorliegen einer Choledocholithiasis bei Cholezystolithiasis [201]; weitere gute diagnostische Indikatoren sind eine sonografische Dilatation der Gallengänge und eine Hyperbilirubinämie [201, 311] (zur Definition hoch- bzw. niedriggradiger Verdacht auf Cholangiolithiasis siehe III.6 und $\odot$ Tab. 4).

Bei zweifelhaftem Befund bzw. möglichen Gallengangssteinen sollten, wenn möglich, die Endosonografie oder alternativ eine MRC vorgeschaltet werden. Sind beide Verfahren zeitnah nicht verfügbar, muss die Entscheidung zur ERC von der Situation des Patienten abhängig gemacht und diese ggf. von einem erfahrenen Untersucher durchgeführt werden. Die höchste Sensitivität für Steine im Ductus hepatocholedochus weist die Endosonografie auf. Sie ist wesentlich risikoärmer als die diagnostische ERC und besitzt bei entsprechender Untersuchererfahrung eine nahezu 100\%ige Sensitivität sowie eine hohe Spezifität (>93\%) zum Nachweis bzw. Ausschluss von Gallengangssteinen, insbesondere auch für kleinere (< $5 \mathrm{~mm}$ ) und präpapilläre Konkremente [298-300, 328-344]. Die Ergebnisse der Endosonografie sind von der Steingröße und dem Gallengangsdurchmesser unabhängig. Die MRC weist insgesamt eine vergleichbare mediane Sensitivität (85 - 93\%) und Spezifität (94-96\%) auf, wobei ihre Aussagefähigkeit für Konkremente $<5 \mathrm{~mm}$ und damit die Beurteilung einer Mikrolithiasis aber eingeschränkt sind [301, 306, 325, 332, 334 - 336, 341, 342, 345 - 372]. Eine kürzlich erschienene Metaanalyse [372] von fünf randomisierten kontrollierten Studien [332, 334-336, 342] mit insgesamt 301 Patienten fand zwischen Endosonografie und MRC keine signifikanten Unterschiede hinsichtlich der Sensitivität (93\% versus 85\%) und Spezifität (96\% versus 93\%) für die Diagnose von Gallengangssteinen.

Zur Darstellung der Anatomie und/oder zum Nachweis von Gallengangssteinen kann auch die intraoperative Cholangiografie durchgeführt werden [107, 373], obgleich deren nicht selektiver Einsatz in Deutschland nicht empfohlen wird [104, 106, 374, 375].

\section{Therapie der Gallengangssteine}

4. Symptomatische Gallengangssteine sind eine Behandlungsindikation (III, starker Konsens, B). Asymptomatische Gallengangssteine können ebenfalls behandelt werden (IV, starker Konsens, C). Kommentar: Die erste Empfehlung wurde von der Konsensuskonferenz in die Kategorie B eingestuft, um aufgrund der Häufigkeit potenziell letaler Komplikationen der symptomatischen Choledocholithiasis eine stärkere als optionale Empfehlung $z u$ geben. Gallengangssteine werden entweder primär im Gallengang gebildet (meist braune Pigmentsteine) oder sind aus der Gallenblase in den Gallengang eingewandert (primäre Cholesterinsteine), wo sie an Größe erheblich zunehmen können (zumeist Pigmentsteine mit einem Cholesterinkern) [376-379]. Die Prävalenz von gleichzeitigen Gallengangssteinen liegt bei symptomatischen Gallenblasensteinen wahrscheinlich zwischen 10 und 20\% [380-384] und bei nicht ikterischen Patienten mit sonografisch nicht erweiterten Gallenwegen unter 10\% [385]. Die Daten zum natürlichen Verlauf von Gallengangssteinen belegen, dass symptomatische Gallengangssteine bei mehr als 50\% der Patienten im Verlauf erneut Koliken verursachen und bei einem Viertel auch Komplikationen nach sich ziehen [326, 376, 386]. Asymptomatische Gallengangssteine scheinen im Verlauf benigner zu sein als 
symptomatische Gallengangssteine. Auch wenn langfristige prospektive Daten bisher fehlen, so lassen kurzfristige Analysen [327, 385,387 ] und langfristige retrospektive Studien [388] den Schluss $z u$, dass weniger als die Hälfte der Patienten mit asymptomatischen Gallengangssteinen symptomatisch werden und mehr als $20 \%$ der Steine spontan abgehen. Daraus ergibt sich, dass asymptomatische Gallengangssteine behandelt werden können, aber nicht zwingend behandelt werden müssen. Spontane Steinpassagen in den Darm sind vermutlich häufig, besonders wenn die Steine klein sind [387, 389]. Stuhluntersuchungen nach ESWL von Gallenblasensteinen haben gezeigt, dass Steine bis zu $8 \mathrm{~mm}$ Durchmesser die Papilla Vateri spontan passieren können [390].

5. Bei cholezystektomierten Patienten mit symptomatischen Gallengangssteinen sollte eine endoskopische Steinextraktion nach Papillotomie vorgenommen werden (Illa, starker Konsens, A). Altersgrenzen für die Papillotomie lassen sich nicht begründen (Ila, starker Konsens, A).

Kommentar: Die endoskopische Papillotomie (EPT; Synonyme: endoskopische Sphinkterotomie, EST oder ES) wurde erstmalig im Jahre 1974 durchgeführt [391]. Ihre Resultate bei der Behandlung von Gallengangssteinen sind mit denen der offenen Gallengangschirurgie vergleichbar [392 -394]. Letalität und Morbidität sind bei der EPT jedoch geringer. Jüngere und ältere Patienten haben nach der derzeitigen Datenlage, auch im Langzeitverlauf, eine ähnliche Komplikationsrate [395-398].

6. Bei Patienten mit gleichzeitig vorliegenden Gallenblasen- und Gallengangssteinen wird ein therapeutisches Splitting empfohlen (III, starker Konsens, C). In Zentren mit hoher Expertise kann eine laparoskopische Cholezystektomie mit laparoskopischer Choledochusrevision erfolgen (Ib, starker Konsens, A).

Kommentar: Als mögliche Optionen bieten sich grundsätzlich sowohl die prä-, die intra- und die postoperative ERC als auch die intraoperative Cholangiografie mit laparoskopischer Gallengangsrevision an [313, 375, 399-410]. Bei sehr hoher Wahrscheinlichkeit einer gleichzeitigen Choledocholithiasis bleibt die präoperative EPT und Steinextraktion die wichtigste Behandlungsoption und wird auch in $>85 \%$ der deutschen Kliniken bevorzugt [411]. Alle Teilnehmer der Konsensuskonferenz sprachen sich für eine Empfehlung zum therapeutischen Splitting aus, auch wenn sich in den letzten Jahren die Datenlage zur laparoskopischen Gallengangsrevision verbessert hat [412]. Die EPT und die Cholezystektomie sollten nicht am selben Tag erfolgen, um postinterventionelle Komplikationen vor Operationsbeginn ausschließen zu können.

Beim primär offenen, konventionellen Vorgehen ist die operative Choledochusrevision in Metaanalysen [412, 413] effizienter als die präoperative ERC und EPT [394, 414-419]. Die Rate an zurückgelassenen Steinen liegt bei 6,7\% [412]. Allerdings gelten schwere Cholangitis und höheres Risikoprofil des Patienten als Einschränkung. Daher sollte immer die individuelle Situation berücksichtigt werden. Anzustreben ist eine möglichst zeitnahe Gesamtbehandlung.

Bei mäßiger Wahrscheinlichkeit einer Choledocholithiasis ist die präoperative ERC nicht Standard, sondern je nach Verfügbarkeit sollten weniger invasive Verfahren vorgeschaltet werden. Sowohl die Endosonografie als auch die MRC besitzen eine hohe Sensitivität und Spezifität zur Entdeckung von Gallengangssteinen [321, 370, 371] (siehe III.3), so dass dann bei positivem Steinnachweis die präoperative EPT mit Gallengangssanierung angestrebt werden kann.
Die intraoperative Gallengangsdarstellung und gegebenenfalls laparoskopische transzystische Gallengangsrevision setzen eine hohe Expertise voraus, die sich darin ausdrückt, dass laparoskopische Gallengangsrevisionen nicht nur fallweise, sondern regelmäßig in mindestens zweistelliger Zahl pro Jahr vorgenommen werden. Als Operationstechniken werden eine transzystische Gallengangsexploration oder eine laparoskopische Choledochotomie durchgeführt. In zwei randomisierten kontrollierten Studien $[420,421]$ und deren Metaanalysen $[412,413]$ unterschieden sich bei 347 Patienten Erfolgsraten, Morbidität und Letalität nicht zwischen therapeutischem Splitting und laparoskopischer Gallengangsrevision, allerdings lagen die Komplikationsraten in diesen Studien bei endoskopischer Intervention mit 13-19\% sehr hoch (16-18\% beim operativen Vorgehen). Bei Patienten mit niedrigem Risiko (ASA I-II ( $\bullet$ Tab. 5) und leichtgradiger Cholangitis) lag die Krankenhaus-Verweildauer bei transzystischer Gallengangsrevision um bis zu 3 Tage niedriger als beim therapeutischen Splitting [420].

\begin{tabular}{|ll|}
\hline Tab.5 & ASA-Klassifikation \\
\hline ASA I & normaler, gesunder Patient \\
\hline ASA II & $\begin{array}{l}\text { Patient mit leichten Allgemeinerkrankungen } \\
\text { ASA III }\end{array}$ \\
$\begin{array}{l}\text { Patient mit schweren Allgemeinerkrankungen und } \\
\text { Leistungsminderung }\end{array}$ \\
\hline ASA IV & $\begin{array}{l}\text { Patient mit einer inaktivierenden Allgemeinerkrankung, } \\
\text { die eine ständige Lebensbedrohung darstellt }\end{array}$ \\
\hline ASA V & $\begin{array}{l}\text { moribunder Patient, von dem nicht erwartet wird, dass er die } \\
\text { nächsten 24 Stunden überlebt, sei es mit oder ohne Inter- } \\
\text { vention }\end{array}$ \\
\hline
\end{tabular}

Die Frage, ob die postoperative ERC der laparoskopischen Gallengangsrevision gleichwertig ist, wurde in zwei weiteren randomisierten kontrollierten Studien bei 166 Patienten untersucht [412, $422,423]$. Vorteil des einzeitigen laparoskopischen Vorgehens sind die kürzere Krankenhaus-Verweildauer und ein geringerer Ressourcenverbrauch [405, 422, 424], nachteilig sind die höhere Rate an Gallenlecks [423] und die eingeschränkte Erfahrung mit diesem Verfahren, für das auch eine entsprechende technische Ausrüstung einschließlich eines flexiblen Choledochoskops erforderlich ist. Aufgrund der wahrscheinlich hohen spontanen Abgangsrate für kleine Gallengangskonkremente [385] (siehe III.4) erscheint zudem ein Verzicht auf eine laparoskopische Gallengangsrevision bzw. eine frühe postoperative ERC bei Steinen < $5 \mathrm{~mm}$ möglich [425].

In mehreren Studien wurde auch die Machbarkeit der intraoperativen ERC demonstriert [402, 408, 410, 426-430]. Allerdings bleibt dieses Verfahren trotz erleichtertem Gallengangszugang über einen durch den Chirurgen transzystisch prograd bis zur Papille eingeführten Draht logistisch sehr aufwendig und kann nicht abschließend beurteilt werden.

Falls eine Choledocholithiasis unwahrscheinlich ist, sind ERC, Endosonografie oder MRC vor einer Cholezystektomie nicht indiziert. Wenn die Wahrscheinlichkeitskriterien eingehalten werden, ist die Routine-Cholangiografie intraoperativ nicht notwendig [431, 432].

Die Kriterien für die Wahrscheinlichkeit einer simultanen Choledocholithiasis sind in $\bigcirc$ Tab. 4 dargestellt. Bei Auswertung der Studien [202, 307-318, 320, 336, 433-436] und auch Metaanalysen [201] ergeben sich drei Konstellationen, die für eine hohe Wahrscheinlichkeit (67-93\%) gleichzeitiger Gallenblasen- und Gallen- 
gangssteine sprechen ( $\bullet$ Tab. 4): Erstens ein sonografisch erweiterter Gallengang ( > 7 - $10 \mathrm{~mm}$ ) [437] bei gleichzeitig erhöhten Bilirubin-, $\gamma$-GT- und ALT-Werten [201, 202], zweitens ein sonografisch > $10 \mathrm{~mm}$ erweiterter Gallengang bei gleichzeitigen Gallenblasensteinen und Koliken und drittens der sonografische Verdacht auf einen Gallengangsstein.

Eine niedrige Wahrscheinlichkeit einer simultanen Cholezystound Choledocholithiasis ergibt sich bei sonografisch normal weiten Gallengängen ohne Erhöhung der Cholestasewerte [321, 438]. Kriterien für eine mäßige Wahrscheinlichkeit ergeben sich dementsprechend dann, wenn weder Kriterien der hohen noch der niedrigen Wahrscheinlichkeit erfüllt werden ( Tab. 4).

7. Falls die endoskopische transpapilläre Therapie nicht möglich ist, bieten sich eine perkutan-transhepatische oder eine chirurgische Behandlung an (III, Konsens, C).

Kommentar: Die perkutan-transhepatische Cholangiografie (PTC) und Steinbehandlung kann bei Misslingen des endoskopisch-retrograden Vorgehens oder bei endoskopisch nicht erreichbarer Papille alternativ zum operativen Vorgehen bei Choledocholithiasis therapeutisch eingesetzt werden [439, 440]. Die Komplikationsraten (Hämobilie, Pneumothorax, Gallenleck in die Bauchhöhle) der interventionell-therapeutisch erweiterten PTC sind höher als die der ERC und EPT [441 -443]. Bei größeren Steinen müssen auch bei der PTC oft adjuvante Lithotripsieverfahren eingesetzt werden [444-446]. Die Option der chirurgischen Choledochusrevision steht in diesen Fällen mindestens gleichwertig zur Verfügung, zumal im Verlauf meistens eine Cholezystektomie indiziert ist.

8. Bei Misslingen (auch unter Einsatz der mechanischen Lithotripsie) der endoskopischen oder perkutan-transhepatischen Steinextraktion sollten als adjuvante Lithotripsieverfahren extrakorporale Stoßwellenlithotripsie (ESWL), intrakorporale Laserlithotripsie oder elektrohydraulische Lithotripsie (EHL) eingesetzt werden (III, starker Konsens, C). Bei gleichzeitiger Cholezystolithiasis sollte die chirurgische Alternative früh erwogen werden (Ib, starker Konsens, A).

Kommentar: Als Maßnahme der Wahl bei großen, endoskopisch primär schwer zu extrahierenden Steinen gilt die mechanische Lithotripsie [447-449]. Als adjuvante Lithotripsieverfahren beim Versagen der mechanischen Lithotripsie wurden die ESWL, die intrakorporale Laserlithotripsie und die EHL entwickelt. Hinsichtlich der Fragmentationseffizienz zeigten kleinere randomisierte kontrollierte Studien diskrete Vorteile der Laserlithotripsie [450, 451]. Die Ergebnisse der EHL in großen Patientenserien aus Zentren sind mit denjenigen der ESWL [452 - 458] und der Laserlithotripsie vergleichbar [459 - 463]. Da sich die adjuvanten Lithotripsieverfahren hinsichtlich ihrer Effizienz und ihrer Komplikationsraten nicht wesentlich unterscheiden, sollten sie je nach lokaler Verfügbarkeit und Erfahrung eingesetzt werden. Die Litholyse von Gallengangssteinen mit über eine nasobiliäre Sonde infundierten Monooctanoin-, MTBE- oder Gallensäuren-/EDTA-Lösungen wird wegen der mäßigen Erfolgs- und der hohen Nebenwirkungsraten [464, 465] nicht empfohlen.

9. Vor Steinextraktion sollte in der Regel eine Papillotomie durchgeführt werden (la, starker Konsens, A).

Kommentar: Nur in Ausnahmefällen kann die Steinextraktion nach endoskopischer Ballondilatation (EBD) erwogen werden, z.B. bei Gerinnungsdefekten, Immundefekten oder schwieriger Anatomie (BII-Magen). Die Erfolgsrate der Steinextraktion ist nach EBD geringer als nach EPT (90,1\% versus 95,3\%), und das Pankreatitisrisiko ist - insbesondere bei jüngeren Patienten mit nicht oder wenig dilatiertem Gangsystem - doppelt so hoch (8,6\% versus 4,3\%) [466 - 478]. Die EPT mit mechanischer Lithotripsie muss zusätzlich sicher beherrscht werden, weil nach EBD häufiger Steinimpaktionen auftreten, die anderweitig nicht zu beheben sind (20,0\% versus $13,3 \%$ ) [467, 469, 473, 475, 479]. Blutungen ( $0,1 \%$ versus $4,8 \%$ ) und Infektionen (2,5\% versus 5,0\%) sind nach EBD seltener als nach EPT [478]. Die Letalitätsraten beider Verfahren unterscheiden sich in der Metaanalyse [478] nicht signifikant (EPT: 0,3\%, EBD: 0,7\%).

10. Bei Hochrisikopatienten mit endoskopisch nicht entfernbaren Gallengangssteinen ist als Primärversorgung die Einlage einer Endoprothese angezeigt (Ib, starker Konsens, A).

Kommentar: Bei Hochrisikopatienten (z.B. ASA IV; $\bigcirc$ Tab. 5) kann alternativ zur Steinextraktion der Gallefluss durch endoskopisch platzierte Gallengangsprothesen wiederhergestellt werden. Hierzu liegen eine randomisierte kontrollierte Studie [480] und mehrere Kohortenstudien vor [481-488], die - zumindest bei Hochrisikopatienten - Vorteile für die vorübergehende Platzierung einer Endoprothese ergaben [489]. Neuere Studien zeigen, dass im weiteren Verlauf möglichst eine definitive endoskopische und/oder chirurgische Versorgung erfolgen sollte [439, 490]. Eine Begleittherapie mit UDCA kann möglicherweise die Steinextraktion zu einem späteren Zeitpunkt erleichtern [483]. Vor allem aufgrund des Risikos von rezidivierenden Cholangitiden im Langzeitverlauf sollte die Indikation zur Einlage einer Gallengangsprothese bei Gallengangssteinen daher auf die o.a. Patienten beschränkt bleiben und interdisziplinär zwischen Gastroenterologen und Chirurgen abgesprochen werden.

11. Nach erfolgreicher endoskopischer oder perkutaner Gallengangssanierung sollte bei Cholezystolithiasis unter Risikoabwägung möglichst innerhalb von 6 Wochen cholezystektomiert werden (Ib, starker Konsens, A). Eine funktionstüchtige steinfreie Gallenblase muss nicht entfernt werden (III, starker Konsens, C).

Kommentar: Bei Cholezystolithiasis, unabhängig davon, ob sich die Gallenblase retrograd bei der ERC darstellt oder nicht, wurde bereits früher aufgrund des erhöhten Risikos von Komplikationen in den Folgejahren (15\% der Patienten entwickeln im weiteren Verlauf von 5 Jahren eine akute Cholezystitis [35]) eine Cholezystektomie angeraten, wenn es die perioperative Risikoabschätzung erlaubt [394, 417]. Neue randomisierte kontrollierte Multicenterstudien [491, 492], die zwischen laparoskopischer Cholezystektomie nach endoskopischer Gallengangssanierung und abwartendem Verhalten verglichen, bestätigen, dass das abwartende Verhalten mit einer deutlich höheren Komplikationsrate (Koliken $20 \%$ versus $5 \%$; Cholezystitis $6 \%$ versus $0 \%$; Cholangitis $15 \%$ versus 6\%) [492] und häufigeren Notfalleingriffen verbunden ist (Notfallcholezystektomie $37 \%$ versus $0 \%$; Notfall-ERC $10 \%$ versus $0 \%$ ) [491]. Auch liegt die Konversionsrate während der laparoskopischen Cholezystektomie zur offenen Operation signifikant höher. Das Studiendesign der europäischen Multicenterstudie war so geplant, dass die Cholezystektomie innerhalb von sechs Wochen nach der endoskopischen Gallengangssanierung erfolgte; in der Wait-andsee-Gruppe lag sie im Median bei 11 Wochen [491]. Es fand die mehrheitliche Zustimmung der Konsensuskonferenz (74\%), dass der Patient vor der Cholezystektomie auch entlassen werden kann.

Eine prospektive japanische Studie bei 1042 Patienten [493] und Verlaufsbeobachtungen bei Patienten mit funktionstüchtiger, 
steinfreier Gallenblase [258] erlauben den Schluss, dass nach erfolgreicher EPT und Steinextraktion aufgrund des auch im Langzeitverlauf sehr geringen Risikos erneuter biliärer Komplikationen ein abwartendes Verhalten gerechtfertigt ist; randomisierte kontrollierte Studien liegen zu dieser Frage jedoch nicht vor.

\section{Therapie der akuten Cholangitis}

12. Die (obstruktive) steinbedingte akute Cholangitis sollte so rasch wie möglich (bei septischen Zeichen notfallmäßig) durch endoskopische Beseitigung des Steines behandelt werden (Ib, starker Konsens, A). Eine antibiotische Begleittherapie ist angezeigt (Ib, starker Konsens, A). Gelingt die Steinentfernung nicht, müssen eine nasobiliäre Sonde oder ein Stent eingelegt werden (IIb, starker Konsens, B). Falls das transduodenale Vorgehen misslingt, ist eine perkutane Drainage angezeigt (Ilb, starker Konsens, B).

Kommentar: Bei der Cholangitis zeigte sich in einer randomisierten kontrollierten Studie ein signifikanter Vorteil des endoskopischen gegenüber dem operativen Vorgehen hinsichtlich der Komplikationen und der Letalität [15]. Deshalb gilt das endoskopische Vorgehen heute nach wie vor als Therapie der Wahl. Falls zunächst keine endoskopische Steinentfernung erfolgt, sind bei drohender mechanischer Obstruktion der Gallenwege Drainagemaßnahmen unerlässlich, wobei nasobiliäre Sonde und Stent gleichwertig sind [484, 494, 495]. Die nasobiliäre Sonde ermöglicht die Gewinnung von Galle für mikrobiologische Untersuchungen und ggf. Spülungen $[14,17]$. Eine systemische antibiotische Therapie soll, ergänzend zur Beseitigung des biliären Abflusshindernisses, septische Komplikationen verhindern und hat sich in mehreren Studien bei Gallengangsobstruktion bewährt [16, 496-498]. Gelingt es auf endoskopischem Weg nicht, die Papilla Vateri oder den Gallengang $z u$ erreichen, ist der perkutan-transhepatische Zugang mit Anlage einer perkutan-transhepatischen Cholangio-Drainage (PTCD) eine Alternative. Große Gallengangssteine können mit den gleichen Fragmentationsmethoden, gegebenenfalls unter Zuhilfenahme der Cholangioskopie, zerkleinert und entfernt werden, wie dies für den transduodenalen Zugang gilt (siehe III.8). Die Erfolgsrate für die Behandlung von Steinen in den Gallengängen mit perkutan-transhepatischer Cholangioskopie beträgt 90 -99\%, die Komplikationsrate ist etwas höher als bei der EPT [442, 443].

In Fällen, in denen die Papille endoskopisch zwar einstellbar, jedoch nicht intubierbar ist, kann ein kombiniertes perkutanes und endoskopisches Vorgehen indiziert sein (so genanntes RendezvousVerfahren), bei dem ein perkutan platzierter Führungsdraht endoskopisch aufgenommen wird und als Schiene für die weitere, dann endoskopische Intervention verwendet werden kann. Kann die steinbedingte Gallengangsobstruktion nicht behoben werden, muss perkutan-transhepatisch interveniert werden. Steht dieses Verfahren nicht zur Verfügung, sollte der Patient in ein Zentrum verlegt werden. Von Teilnehmern der Konsensuskonferenz wurde darauf hingewiesen, dass nach Versagen der endoskopischen Therapie alternativ zur perkutanen Intervention auch eine operative Revision erwogen werden kann. Es bestand kein Konsens darüber, ob bei steinbedingter eitriger Cholangitis auch nach Steinentfernung vorübergehend die Anlage einer nasobiliären Sonde erfolgen sollte.

\section{ERC/Papillotomie bei biliärer Pankreatitis}

13. Bei der biliären Pankreatitis mit Cholestase/Ikterus und/oder Zeichen einer Cholangitis ist eine ERC/Papillotomie mit Steinextraktion nach Maßgabe der klinischen Dringlichkeit so rasch wie möglich, bei Cholangitis innerhalb von 24 Stunden nach Aufnahme, indiziert (la, starker Konsens, A).
Kommentar: Beim Vorliegen einer Cholangitis oder schweren biliären Pankreatitis ist der Wert einer möglichst raschen endoskopischen Intervention gesichert ( Tab. 6) [499-502]. In einer aktuellen Studie [503], die frühe und elektive ERC verglich und die in der Metaanalyse [502] noch nicht berücksichtigt wurde, wurden bei 72\% der Patienten im ERC-Arm Gallengangssteine nachgewiesen und entfernt; Patienten mit Cholangitis waren ausgeschlossen, und $>90 \%$ der Patienten wurden im Verlauf des stationären Aufenthaltes cholezystektomiert. Es ergaben sich keine Unterschiede hinsichtlich Organversagen, Komplikationen und Mortalität [503]. Es bestand in Anlehnung an Studien [15, 500, 504] Konsens, dass die ERC/EPT innerhalb von 24 Stunden nach Aufnahme erfolgen sollte. Der Schweregrad der akuten Pankreatitis wird nach der Atlanta-Klassifikation definiert [505, 506].

14. Bei unkomplizierter biliärer Pankreatitis und abklingender Cholestase/Pankreatitis kann auf eine ERC verzichtet werden (Ib, Konsens, A). Insbesondere kann von einer ERC abgesehen werden, wenn Endosonografie oder MRC keinen Steinnachweis ergaben (IV, Konsens, C).

Kommentar: Bei leichter biliärer Pankreatitis ist keine umgehende endoskopische Intervention erforderlich [501, 502, 504, 507], zumal > 80\% der Steine innerhalb einer Woche spontan abgehen [508, 509]. Dennoch gilt auch für die leichte biliäre Pankreatitis, dass eine Choledocholithiasis beseitigt werden sollte, um ein Pankreatitisrezidiv zu verhindern. Zum genauen Zeitpunkt der Intervention liegen keine ausreichenden Daten vor [510]. Falls die Patienten nach leichter biliärer Pankreatitis cholezystektomiert werden (siehe III.15), muss keine präoperative ERC durchgeführt werden. Es sollten jedoch weitere bildgebende Verfahren (Endosonografie, MRC, intraoperative Cholangiografie) je nach individueller Situation erwogen werden.

Bei fraglicher Indikation zur ERC (sonografisch kein eindeutiger Nachweis von Gallengangssteinen, Fehlen einer Cholangitis) oder bei unklarer Ätiologie wird die Durchführung einer Endosonografie oder MRC empfohlen. Die Datenlage lässt keine eindeutige Überlegenheit einer der beiden Methoden erkennen, sodass die Methodenwahl von den lokalen Gegebenheiten abhängt [300, 323, 325, 511-514]. Ziel der weiterführenden Diagnostik durch Endosonografie oder MRC muss es auch sein, eine andere Ursache der Pankreatitis im Bereich der Papille oder der Pankreasgänge (z.B. einen Tumor) auszuschließen.

15. Bei gegebener Indikation zur Cholezystektomie sollte diese erst nach Abklingen der Pankreatitis erfolgen (III, starker Konsens, C).

Kommentar: Nach Möglichkeit sollte die (laparoskopische) Cholezystektomie (ggf. mit intraoperativer Cholangiografie) nach Abklingen der Pankreatitis erfolgen; dieses Vorgehen vermindert die Rate an septischen Komplikationen [515, 516]. Falls der klinische Verlauf es zulässt, sollte die Cholezystektomie im Rahmen desselben stationären Aufenthaltes erfolgen [517 - 521], da die Wartezeit auf eine elektive Cholezystektomie mit einem signifikanten Risiko für ein Pankreatitisrezidiv einhergeht $[522,523]$. Bei peripankreatischem Flüssigkeitsverhalt oder Pseudozysten wurde ein Zeitraum von 6 Wochen vorgeschlagen, nach dem die Cholezystektomie erst sicher durchgeführt werden kann [516].

Falls eine EPT wegen der biliären Pankreatitis durchgeführt wurde, verhindert diese sehr wahrscheinlich eine erneute biliäre Pankreatitis oder verringert zumindest das Pankreatitisrisiko erheblich [523 - 526], so dass die Indikation zur Cholezystektomie nach EPT nur mit den möglichen Komplikationen vonseiten der Gallen- 
Tab. 6 Randomisierte kontrollierte Studien zur endoskopischen Intervention bei akuter biliärer Pankreatitis (Abkürzungen: ARR, absolute Risikoreduktion; NNT, Number needed to treat; RRR, relative Risikoreduktion)

\begin{tabular}{|c|c|c|c|c|c|c|c|c|c|c|c|c|}
\hline Studie & $\begin{array}{l}\text { Inter- } \\
\text { vention }\end{array}$ & $\mathrm{n}$ & $\begin{array}{l}\text { Zeit bis } \\
\text { zur ERC } \\
\text { (h) }\end{array}$ & $\begin{array}{l}\text { Schweregrad } \\
\text { der Pankrea- } \\
\text { titis } \\
\text { (\%) }\end{array}$ & $\begin{array}{l}\text { Steine } \\
(\%)\end{array}$ & $\begin{array}{l}\text { Erfolgsrate/ } \\
\text { ERC-Rate } \\
\text { (\%) }\end{array}$ & $\begin{array}{l}\text { Kompli- } \\
\text { kationen } \\
(\%)\end{array}$ & $\begin{array}{l}\text { RRR } \\
\text { (\%) }\end{array}$ & NNT & $\begin{array}{l}\text { Morta- } \\
\text { lität } \\
(\%)\end{array}$ & $\begin{array}{l}\text { RRR } \\
\text { (\%) }\end{array}$ & NNT \\
\hline $\begin{array}{l}\text { Neoptolemos } \\
\text { et al. } 1988 \\
\text { [499] }\end{array}$ & $\begin{array}{l}\text { früh } \\
\text { selektiv }\end{array}$ & $\begin{array}{l}59 \\
62\end{array}$ & $\begin{array}{l}<72 \\
>120\end{array}$ & $\begin{array}{l}44 \text { (modif. } \\
\text { Glasgow) }\end{array}$ & 85 & $\begin{array}{l}90 \\
23\end{array}$ & $\begin{array}{l}16,9 \\
33,9\end{array}$ & 50 & 6 & $\begin{array}{l}1,7 \\
8,1\end{array}$ & 79 & 16 \\
\hline $\begin{array}{l}\text { Fan et al. } 1993 \\
\text { [500] }\end{array}$ & $\begin{array}{l}\text { früh } \\
\text { selektiv }\end{array}$ & $\begin{array}{l}97 \\
98\end{array}$ & $<24$ & $\begin{array}{l}42 \\
\text { (Ranson) }\end{array}$ & 38 & $\begin{array}{l}90 \\
28\end{array}$ & $\begin{array}{l}17,5 \\
28,6\end{array}$ & 39 & 9 & $\begin{array}{l}5,2 \\
9,2\end{array}$ & 44 & 25 \\
\hline $\begin{array}{l}\text { Fölsch et al. } \\
1997 \text { [501] }\end{array}$ & $\begin{array}{l}\text { früh } \\
\text { selektiv }\end{array}$ & $\begin{array}{l}126 \\
112\end{array}$ & $<72$ & $\begin{array}{l}22 \text { (modif. } \\
\text { Glasgow) }\end{array}$ & 46 & $\begin{array}{l}95 \\
20\end{array}$ & $\begin{array}{l}46,0 \\
50,9\end{array}$ & 20 & 20 & $\begin{array}{r}11,1 \\
6,3\end{array}$ & -77 & -21 \\
\hline $\begin{array}{l}\text { Oria et al. } \\
2007 \text { [503] }\end{array}$ & $\begin{array}{l}\text { früh } \\
\text { selektiv }\end{array}$ & $\begin{array}{l}51 \\
51\end{array}$ & $<72$ & $\begin{array}{l}36 \\
\text { (APACHE II) }\end{array}$ & 72 & $\begin{array}{r}97 \\
4\end{array}$ & $\begin{array}{l}21,6 \\
17,6\end{array}$ & -19 & -25 & $\begin{array}{l}5,9 \\
2,0\end{array}$ & -195 & -26 \\
\hline
\end{tabular}

blasensteine zu begründen ist. Zu dieser Problematik fehlen ausreichende prospektive Daten. Bei älteren Patienten und/oder hohem Operationsrisiko (z.B.ASA IV; $\bullet$ Tab. 5) kann von der Cholezystektomie auch bei Gallenblasensteinen abgesehen werden, wenn eine EPT durchgeführt wurde [523-526].

Bei „idiopathischer“ akuter Pankreatitis ist nach Ausschluss anderer Ursachen der Pankreatitis (Alkohol, Stoffwechselstörungen, hereditäre Pankreatitis) die Cholezystektomie auch bei sonografisch steinfreier Gallenblase als Therapieoption erwogen worden, da eine Mikrolithiasis ursächlich zugrunde liegen könnte [339, 343, 527-529]. Obgleich auch durch UDCA (10 mg/kg/d) das Pankreatitisrisiko in dieser Situation gesenkt wird [527, 530], hat dieses Vorgehen den Nachteil einer lebenslangen Behandlung. Es kommt insbesondere bei älteren Patienten mit hohem Operationsrisiko infrage. Ob bei asymptomatischen kleinen Gallenblasensteinen ( $<5 \mathrm{~mm}$ ), für die ebenfalls ein höheres Pankreatitisrisiko als bei großen Steinen besteht [531], eine prophylaktische Cholezystektomie sinnvoll ist [532], müsste durch eine prospektive Studie geklärt werden. Ohne solche Daten gilt auch in dieser Situation, dass asymptomatische Gallenblasensteine nicht behandelt werden.

\section{Diagnostik und Therapie bei intrahepatischen Gallen- steinen}

16. Bei Verdacht auf intrahepatische Gallensteine ist die transkutane B-Mode-Sonografie die diagnostische Methode der ersten und die MRC die Methode der zweiten Wahl (III, Konsens, C).

17. Asymptomatische intrahepatische Steine müssen nicht grundsätzlich behandelt werden (III, Konsens, C). Bei symptomatischen intrahepatischen Steinen sollte die Therapieentscheidung patientenbezogen interdisziplinär getroffen werden (IV, starker Konsens, C).

Kommentar: Die Sonografie hat gegenüber der diagnostisch intendierten ERC Vorteile, da bei dieser lithogene Abbrüche der intrahepatischen Gallenwege durch die meist nicht kalzifizierten Konkremente in aller Regel nicht in Erscheinung treten. Darüber hinaus birgt die ERC die Gefahr der Cholangitisinduktion, so dass nicht invasive Verfahren zur Diagnostik vorzuziehen sind. Die Sonografie erlaubt zuverlässig die Darstellung der Steine und die Diagnose prästenotischer Erweiterungen der Gallengänge. Die MRC ist ebenfalls der ERC bei der Diagnostik der intrahepatischen Steine überlegen (Sensitivität 97\% versus 59\%); auch intrahepatische biliäre Strikturen werden zuverlässig entdeckt (Spezifität 97\%, Sensitivität 93\%) [364, 533, 534]. Mittels CT können dilatierte Gänge und Strikturen sowie Leberabszesse dargestellt werden, obgleich die Steine häufig nicht direkt zur Darstellung kommen [534].
Die ERC und PTC haben ihre primäre Bedeutung für die anschließende Therapieplanung. Asymptomatische intrahepatische Steine werden im Verlauf von 15 Jahren nur bei 11,5\% der Patienten im Mittel nach 3,4 Jahren symptomatisch. Die häufigsten Symptome sind Koliken, Cholangitis und Leberabszess, seltener Cholangiokarzinome [535]. Aus diesem Grund ist das abwartende Verhalten gerechtfertigt. Bei symptomatischen Steinen ist die interdisziplinäre Therapieplanung sinnvoll [536]. Der Langzeiterfolg der Chirurgie ist insbesondere dann gegeben, wenn der Befall auf einzelne periphere Lebersegmente und einen Leberlappen begrenzt ist [537]. Bei diffuser Verteilung der intrahepatischen Steine sind modifizierte perkutan-transhepatische cholangioskopische Verfahren mit Lithotripsie und Gallengangsdilatationen sinnvoll [534, 538-542].

\section{Therapie in der Schwangerschaft}

18. Symptomatische Gallengangssteine werden in der Gravidität primär durch endoskopische Papillotomie und Steinextraktion therapiert (III, starker Konsens, C). Falls eine Verwendung von Röntgenstrahlen erforderlich ist, stellt diese bei Beachtung der Strahlenschutzrichtlinien auch im ersten Trimenon keine absolute Kontraindikation dar (IV, starker Konsens, C).

Kommentar: Mehrere Studien haben die Sicherheit der ERC in der Schwangerschaft nachgewiesen [543-547]. Die Untersuchung sollte durch einen erfahrenen Untersucher erfolgen, wobei eine Ultraschall-geleitete EPT helfen kann, die Strahlenbelastung vollständig zu vermeiden [548]. Die Ermittlung der Strahlendosis basiert auf dem 3-Stufen-Konzept, wobei bis $20 \mathrm{mSv}$ (Stufe I) eine Dosisabschätzung und Protokollierung durch den Arzt ausreichend ist [549]. Da eine Durchleuchtung a.p. bei normaler Konstitution zu einer Uterusdosis von $24 \mathrm{mSv} / \mathrm{min}$ führt, sollten die Durchleuchtungszeiten möglichst kurz gehalten und keine Röntgenaufnahmen angefertigt werden [549]. Eine schwangere Patientin sollte während der ERC auf die linke Körperseite gelagert werden, um eine Hypotonie in Rückenlage und eine Okklusion der Vena cava zu vermeiden. Bei der EPT ist zu beachten, dass der Uterus nicht zwischen Sphinkterotom und Elektrode liegt.

\section{Prävention von Gallengangssteinen}

19. Es gibt keine gesicherte medikamentöse Prävention der Entstehung von Rezidivsteinen in den Gallengängen (III, starker Konsens, C).

Kommentar: Rezidivsteine im Gallengang sind zumeist Pigmentsteine, mit hervorgerufen durch bakterielle Besiedelung der Gallengänge. Sie werden bei 5-20\% der Patienten nach EPT beob- 
achtet und können im Regelfall erneut endoskopisch entfernt werden [550-555]. Eine gesicherte prophylaktische Therapie besteht derzeit nicht. Erste Arbeiten zeigten keinen Nutzen einer medikamentösen Prophylaxe. Ältere, nicht überprüfte Daten zu einer UDCA-Behandlung zeigten bei einer kleinen Patientengruppe und kurzem Follow-up eine leichte Reduktion der Rezidivrate [556].

\section{Qualitätssicherung}

\section{$\nabla$}

1. Cholezystektomien, die unter der Hauptdiagnose einer nicht bösartigen Erkrankung der Gallenblase oder der Gallengänge oder einer akuten Pankreatitis durchgeführt werden, sind dokumentationspflichtig (Bundesgeschäftsstelle Qualitätssicherung, BQS) (IV, starker Konsens, A).

Kommentar: Diese Empfehlung wurde von der Konsensuskonferenz in die Kategorie A eingestuft, da eine Dokumentationspflicht besteht, die auf der externen vergleichenden Qualitätssicherung der deutschen Krankenhäuser nach § 137 SGB V basiert. Die Daten werden von der Bundesgeschäftsstelle Qualitätssicherung (http//www.bqs-online.de/) zentral erfasst und jährlich ausgewertet ( Tab. 7). Cholezystektomien, die im Rahmen größerer abdomineller Eingriffe durchgeführt werden, fallen nicht hierunter; die Aufnahme dieser Cholezystektomien in die Qualitätssicherung wird im Rahmen des BQS-Verfahrens zur Diskussion gestellt.

2. Die folgenden Qualitätsindikatoren sollten für die Cholezystektomie erfüllt werden (Zielbereiche in Klammern):

- Erhebung eines histologischen Befundes (100\%)

- Durchführung präoperativer Diagnostik bei Zeichen der extrahepatischen Cholestase (100\%)

- Rate eingriffsspezifischer behandlungsbedürftiger Komplikationen (Blutung, periphere Gallengangsverletzung, Okklusion oder Durchtrennung des Ductus hepatocholedochus, Residualstein im Gallengang) ( $\leq$ 95\%-Perzentile aller Kliniken)

- Okklusion oder Durchtrennung des Ductus hepatocholedochus (Sentinel Event, siehe Begriffsdefinitionen)

- Rate allgemeiner behandlungsbedürftiger postoperativer Komplikationen ( $\leq \mathbf{9 5} \%$-Perzentile aller Kliniken)

- Reinterventionsrate bei laparoskopisch begonnener Cholezystektomie ( $\leq \mathbf{2} \%$ )

- Letalität in den ASA-Risikoklassen I-III (Sentinel Event)
(IV, starker Konsens, C).

Kommentar: Bezüglich der Heranziehung der Konversionsrate bei laparoskopisch begonnener Cholezystektomie und der Rate fraglicher Indikationen als Qualitätsindikatoren konnte kein Konsens erzielt werden. Die Erfassung der stationären Wiederaufnahmerate als Qualitätsparameter ist aufgrund mangelnder Verfügbarkeit von Daten derzeit nicht möglich. Die Letalität aller in der BQS-Erhebung 2005 erfassten Cholezystektomien lag bei 0,66\%; bei Patienten der Risikoklassen ASA I-III ( $\bullet$ Tab. 5) betrug sie 0,39\% ( Tab. 7) [5, 147].

3. Gallenblasenkarzinome sollten dem zuständigen Krebsregister bzw. Tumorzentrum gemeldet werden (IV, starker Konsens, C). Kommentar: Im Rahmen der Cholezystektomie, die ursprünglich durch das Gallensteinleiden indiziert war, kommt es relativ häufig zur Entdeckung vormals okkulter Gallenblasenkarzinome. Im Falle eines präoperativ nicht diagnostizierten Gallenblasenkarzinoms besteht die Möglichkeit, dieses zusätzlich im Zentralregister für okkulte Gallenblasenkarzinome der Deutschen Gesellschaft für Chirurgie (DGCH) zu melden (http://www.ketteler-krankenhaus.de/register/infos.htm) [557]. Diese freiwillige Meldung entspricht einer weit verbreiteten Praxis (bis dato mehr als 500 gemeldete Fälle) und wird daher dem Operateur nahe gelegt.

4. Die Teilnahme am Deutschen Papillotomieregister wird dringend empfohlen (IV, starker Konsens, C).

Kommentar: Das Deutsche Papillotomieregister ist eine externe Maßnahme zur Sicherung der Ergebnisqualität der EPT mittels prospektiver Datenerhebung (http://www.papillotomieregister.de/). Es ist ein Projekt der Arbeitsgemeinschaft leitender gastroenterologischer Krankenhausärzte (ALGK) und der DGVS. Die Pilotphase (Machbarkeitsstudie) hat belegt, dass relevante Daten, einschließlich technischer Details zum Vergleich der Ergebnisse unterschiedlicher Endoskopieeinheiten, unter den Bedingungen der täglichen Praxis dokumentiert werden [558]. Ziele sind die wissenschaftlich fundierte Dokumentation bisher nicht ausreichend abgesicherter Faktoren der Ergebnisqualität der EPT und die kontinuierliche Verbesserung der Struktur-, Prozess- und Ergebnisqualität der EPT. Die Daten zur Ergebnisqualität der EPT sollen Rückschlüsse auf Faktoren der Prozessqualität erlauben und Maßnahmen zur Qualitätsverbesserung initiieren.

\begin{tabular}{lll}
\hline Prozessqualität & & \\
\hline präoperative Diagnostik bei extrahepatischer Cholestase & $14083 / 14817$ & $95,05 \%$ \\
\hline Erhebung eines histologischen Befundes & $163623 / 167263$ & $97,82 \%$ \\
\hline Ergebnisqualität & & $3,69 \%$ \\
\hline eingriffsspezifische Komplikationen & $6333 / 171611$ & $0,15 \%$ \\
- Okklusion oder Durchtrennung des Ductus hepatocholedochus & $264 / 171611$ & $0,34 \%$ \\
- periphere Gallengangsverletzung & $591 / 171611$ & $0,53 \%$ \\
- Residualstein im Gallengang & $908 / 171611$ & $1,21 \%$ \\
- Blutung & $2083 / 171611$ & \\
postoperative Wundinfektionen & & $1,53 \%$ \\
- alle Patienten & $2625 / 171611$ & $0,65 \%$ \\
- Patienten der ASA-Risikoklasse 0 & $656 / 100472$ & $3,75 \%$ \\
\hline allgemeine postoperative Komplikationen & $6443 / 171611$ & \\
Reinterventionsrate & & $2,04 \%$ \\
- alle Patienten & $3509 / 171611$ & $1,68 \%$ \\
- Patienten mit laparoskopisch begonnener Operation & $2613 / 155907$ & \\
Letalität & & $0,66 \%$ \\
- alle Patienten & $1135 / 171611$ & $0,39 \%$ \\
- Patienten der ASA-Risikoklassen I-III & $664 / 169135$ & $0,27 \%$ \\
- Patienten mit laparoskopisch begonnener Operation & $426 / 155907$ &
\end{tabular}

Tab.7 Qualitätsindikatoren der Cholezystektomie $2005[5,147]$ 
5. Qualitätsindikatoren für die EPT sind: Erfolgsrate der Steinextraktion, Komplikationen und Letalität (IV, starker Konsens, C). Kommentar: Die Erfolgs-, Komplikations- und Letalitätsraten der EPT haben sich seit ihrer Einführung nicht nennenswert geändert. Bei mindestens 85\% der Patienten lassen sich die Gallengangssteine nach EPT endoskopisch entfernen [558]. Bei Einsatz von intra- und extrakorporaler Lithotripsie kann die Erfolgsrate auf $>95 \%$ gesteigert werden [478]. Die wesentlichen durch die EPT hervorgerufenen Komplikationen umfassen Pankreatitis (1,3-6,7\%), Blutung (0,7-2,4\%), Cholangitis und Sepsis (0,1-5,0\%) sowie Perforation (0,3-1,1\%) [478, 559-566]. Komplikationen treten häufiger bei Duodenaldivertikel [567], Leberzirrhose [559] oder Dysfunktion des Sphincter Oddi auf [559, 564]. Alter < 60 Jahre [559, 561, 563, 565, 568, 569], weibliches Geschlecht [559], eine lange Dauer der Intervention, die schwierige Kanülierung des Gallengangs, die Precut-Sphinkterotomie und die Kombination von perkutanen und transduodenalen Interventionen erhöhen das Risiko für das Auftreten von Komplikationen [559, 564]. Erfahrene Endoskopiker mit einer Frequenz von mehr als einer EPT pro Woche verursachen signifikant weniger Komplikationen [559, 561]. Die Letalität der EPT beträgt in großen Serien zwischen 0,2 und 5,8\% [565]; bei ausschließlicher Berücksichtigung von Gallensteinen als Indikation liegt sie mit etwa 0,2\% am unteren Rand dieses Bereiches und sollte daher als Sentinel Event (siehe Begriffsdefinitionen) angesehen werden.

6. Die endoskopische Papillotomie bei Choledocholithiasis kann bei ausgewählten Patienten ambulant durchgeführt werden (IIb, starker Konsens, B). Bei der Entscheidung zur ambulanten EPT sind prozedurbezogene, patientenindividuelle und soziale Umfeldfaktoren bedeutend (IV, starker Konsens, C).

Kommentar: Mehrere Studien haben gezeigt, dass Patienten unter bestimmten Voraussetzungen direkt nach einer ERC und sogar nach EPT entlassen werden können. In der Regel wird eine stationäre Nachbeobachtung von 24 h empfohlen, da die meisten Komplikationen nach EPT erst nach 4- 24 h auftreten [570, 571]. In einer prospektiven Multicenterstudie betrug die stationäre Wiederaufnahmerate nach ambulanter EPT 5,7\% [571]. Bei Vorliegen spezifischer Risikofaktoren (Sphincter-Oddi-Dysfunktion, jüngeres Lebensalter, schwierige Kanülierung, Precut-Sphinkterotomie, Pankreasgangdarstellung, kombiniertes endoskopisch-perkutanes Vorgehen, Leberzirrhose; siehe III.5) stieg diese Rate auf 12,2\% an, wohingegen sie ohne diese Risikofaktoren bei 4,2\% lag [571].

7. Outcome-Parameter nach Cholezystektomie sind: Komplikationen, Arbeitsfähigkeit und Lebensqualität (IV, starker Konsens, C). Kommentar: Diese Outcome-Parameter wurden überwiegend im frühpostoperativen Verlauf untersucht (siehe II.7), während für Langzeitergebnisse bisher keine ausreichenden Daten vorliegen. Patienten nach laparoskopischer Cholezystektomie haben weniger Schmerzen, eine kürzere Krankenhaus-Verweildauer und eine raschere Rekonvaleszenz als nach offener Cholezystektomie [108, 572]. Zukünftige Studien sollten vermehrt die perioperative Rehabilitation und den Ressourceneinsatz berücksichtigen. In den letzten Jahren wurden mehrere Studien zur Verbesserung der Lebensqualität nach laparoskopischer Cholezystektomie veröffentlicht, wobei am häufigsten der Gastrointestinal Quality of Life Index (GIQLI) von Eypasch et al. [573] verwendet wurde [574-581]. Es liegen zwei randomisierte kontrollierte Studien zum Vergleich der Lebensqualität nach laparoskopischer und offener Cholezystektomie vor, wobei eine signifikante Verbesserung bereits 10 Tage nach laparoskopischer, aber erst 1 Monat nach of- fener Cholezystektomie festgestellt wurde $[156,159]$. Obgleich das Outcome insbesondere für symptomatische Patienten mit niedrigem Operationsrisiko gut ist [581], profitieren auch ältere und komorbide Patienten von der laparoskopischen Operation, wenn keine lokalen oder anästhesiologischen Kontraindikationen vorliegen [572, 579]. Gallengangsverletzungen (siehe II.7) führen in prospektiven Studien trotz sehr guter objektiver Ergebnisse der überwiegend endoskopischen Therapie zu einer signifikanten Beeinträchtigung der Lebensqualität im Langzeitverlauf [159].

8. Die elektive laparoskopische Cholezystektomie kann bei ausgewählten Patienten ambulant durchgeführt werden (Ib, starker Konsens, A). Bei der Entscheidung zur ambulanten laparoskopischen Cholezystektomie sind prozedurbezogene, patientenindividuelle und soziale Umfeldfaktoren bedeutend (IV, starker Konsens, C).

Kommentar: $\mathrm{Zu}$ dieser Fragestellung liegen mehrere randomisierte kontrollierte Studien vor [582 - 584]. In der gut geplanten, randomisierten kontrollierten Studie aus Schweden [584] unterschieden sich Komplikationsraten und Lebensqualitätsindex nicht zwischen ambulanten Patienten und stationären Kurzliegern $(n=100)$; die Kosten wurden kaum gesenkt. Zwischen 1993 und 1997 stieg der Anteil ambulanter Cholezystektomien in einer retrospektiven amerikanischen Studie von 0,6\% auf 48\% [585]. Abhängig von den Studienkriterien kamen 40 - 60\% aller Elektivpatienten für eine tageschirurgische Behandlung in Betracht [586-588]. Bei etwa 80\% der Patienten ist die Durchführung dann tatsächlich ambulant möglich; die Wiederaufnahmerate ist mit 3,4\% vertretbar gering [589]. Beachtenswert ist allerdings, dass ein hoher Anteil der Patienten (20-32\%) retrospektiv eine stationäre Behandlung vorgezogen hätte [586, 590, 591]. Das Konsensus-Statement wurde von den an der Konsensuskonferenz teilnehmenden Chirurgen einstimmig befürwortet. Voraussetzung für eine ambulante Cholezystektomie sind neben patientenspezifischen Faktoren eine sorgfältige präoperative Vorbereitung und Aufklärung sowie strukturierte Nachsorgepläne.

\section{Addendum A: Klassifikation der Leitlinien (0 Tab. 8-10) \\ 7}

Tab. 8 Klassifikation der Evidenzstärke

la systematisches Review oder Metaanalyse randomisierte kontrollierter Studien

Ib mindestens eine gut geplante, randomisierte kontrollierte Studie

Ila mindestens eine gut geplante, kontrollierte Studie ohne Randomisierung (z. B. Fallkontrollstudie oder historischer Vergleich)

IIb mindestens eine gut geplante, quasi experimentelle Studie (z. B. Fallkontrollstudie oder historischer Vergleich)

III gut geplante, nicht experimentelle deskriptive Studien (Fallserien, Korrelationsstudien)

IV Berichte/Meinungen von Expertenkreisen, Konsensuskonferenzen und/oder klinische Erfahrung ohne explizite kritische Bewertung (physiologische Modelle, Vergleiche oder Grundsätze)

Tab.9 Klassifikation der Konsensstärke

\begin{tabular}{|ll}
\hline starker Konsens & Zustimmung von $\geq 95 \%$ der Teilnehmer \\
\hline Konsens & Zustimmung von 75-95\% der Teilnehmer \\
\hline $\begin{array}{l}\text { mehrheitliche } \\
\text { Zustimmung }\end{array}$ & Zustimmung von 50-75\% der Teilnehmer \\
\hline kein Konsens & Zustimmung von < 50\% der Teilnehmer \\
\hline
\end{tabular}


Tab.10 Klassifikation der Konsensusempfehlungen

\begin{tabular}{|c|c|}
\hline A & $\begin{array}{l}\text { starke Empfehlung } \\
\text { Evidenzstärke la + lb/starker Konsens oder Konsens/direkte } \\
\text { Anwendbarkeit } \\
\text { Evidenzstärke Ila/starker Konsens/direkte Anwendbarkeit }\end{array}$ \\
\hline B & $\begin{array}{l}\text { Empfehlung } \\
\text { alle unter A, jedoch mit extrapolierter Anwendbarkeit } \\
\text { Evidenzstärke la + Ib/mehrheitliche Zustimmung/direkte } \\
\text { Anwendbarkeit } \\
\text { Evidenzstärke Ila/Konsens/direkte Anwendbarkeit } \\
\text { Evidenzstärke IIb/starker Konsens bzw. Konsens/direkte } \\
\text { Anwendbarkeit }\end{array}$ \\
\hline C & $\begin{array}{l}\text { optional } \\
\text { alle unter B, jedoch mit extrapolierter Anwendbarkeit } \\
\text { Evidenzstärke la + Ib/kein Konsens/direkte Anwendbarkeit } \\
\text { Evidenzstärke Ila + IIb/mehrheitliche Zustimmung/direkte } \\
\text { Anwendbarkeit } \\
\text { Evidenzstärke III/starker Konsens bis mehrheitliche Zustimmung/ } \\
\text { direkte Anwendbarkeit } \\
\text { Evidenzstärke IV/starker Konsens bzw. Konsens/direkte } \\
\text { Anwendbarkeit }\end{array}$ \\
\hline
\end{tabular}

\section{Addendum B: Zeitlicher Ablauf der Leitlinien- erstellung ( $O$ Tab. 11$)$}

$\nabla$

Tab. 11 Zeitlicher Ablauf der Leitlinienerstellung

\begin{tabular}{|c|c|c|}
\hline Aufgabe & Teilaufgabe & Zeitraum \\
\hline \multirow[t]{5}{*}{ Initiierung } & $\begin{array}{l}\text { Auftrag der Leitlinienkom- } \\
\text { mission der DGVS }\end{array}$ & $5 / 05$ \\
\hline & $\begin{array}{l}\text { Festlegung der zu beteiligen- } \\
\text { den Gesellschaften und Aus- } \\
\text { wahl der Konferenzteilneh- } \\
\text { mer }\end{array}$ & $6-7 / 05$ \\
\hline & $\begin{array}{l}\text { Anmeldung des Leitlinien- } \\
\text { projekts bei der AWMF }\end{array}$ & $7 / 05$ \\
\hline & Erstellung von Suchbegriffen & $8 / 05$ \\
\hline & $\begin{array}{l}\text { systematische Literatur- } \\
\text { recherche und -bewertung }\end{array}$ & $9 / 05-2 / 06$ \\
\hline \multirow[t]{4}{*}{ Delphi-Befragung } & Erstellung des Fragebogens & $1 / 06$ \\
\hline & $\begin{array}{l}\text { E-mail-basierter Versand der } \\
\text { Fragebögen und der aktuel- } \\
\text { len Literatur (Volltext) }\end{array}$ & $2 / 06$ \\
\hline & $\begin{array}{l}\text { E-mail-basierte Beantwor- } \\
\text { tung der Fragebögen }\end{array}$ & $3 / 06$ \\
\hline & $\begin{array}{l}\text { Excel-basierte Auswertung } \\
\text { der Fragebögen }\end{array}$ & $3-4 / 06$ \\
\hline \multirow[t]{3}{*}{ Konsensuskonferenz } & $\begin{array}{l}\text { Erarbeiten von Vorschlägen } \\
\text { für die Konsensusempfehlun- } \\
\text { gen in den Arbeitsgruppen- } \\
\text { sitzungen }\end{array}$ & $4 / 06$ \\
\hline & $\begin{array}{l}\text { Diskussion der Leitlinie mit } \\
\text { Patientenvertretern }\end{array}$ & \\
\hline & $\begin{array}{l}\text { Plenarsitzung mit Abstim- } \\
\text { mung }\end{array}$ & \\
\hline \multirow[t]{5}{*}{ Nachbereitung } & $\begin{array}{l}\text { Erstellung und Versand des } \\
\text { Leitlinienentwurfs }\end{array}$ & $5-8 / 06$ \\
\hline & $\begin{array}{l}\text { Überarbeitung und Verfassen } \\
\text { der Kommentare durch die } \\
\text { Arbeitsgruppenvorsitzenden }\end{array}$ & $9-12 / 06$ \\
\hline & $\begin{array}{l}\text { Versand der editierten Manu- } \\
\text { skripte an alle Teilnehmer }\end{array}$ & $1 / 07$ \\
\hline & Endfassung der Leitlinie & $2 / 07$ \\
\hline & $\begin{array}{l}\text { Vorlage bei der Leitlinien- } \\
\text { kommission der DGVS }\end{array}$ & $2 / 07$ \\
\hline
\end{tabular}

\section{Addendum C: Suchbegriffe für die MEDLINE- Literaturrecherche \\ $\nabla$}

biliary pancreatitis OR gallstone pancreatitis chenodeoxycholic acid AND (gallstone OR cholelithiasis) cholangiopancreatography OR ERCP AND (gallstone OR cholelithiasis)

cholecystectomy AND (clinical trial OR controlled clinical trial OR randomized controlled trial)

cholecystitis AND (clinical trial OR controlled clinical trial OR randomized controlled trial)

cholecystostomy AND (gallstone OR cholelithiasis)

choledocholithiasis OR bile duct stone OR bile duct calculi

endosonography OR endoscopic ultrasound AND (gallstone OR cholelithiasis)

extracorporeal AND (gallstone OR cholelithiasis)

intraoperative cholangiography OR intraoperative cholangiogram AND (gallstone OR cholelithiasis)

lithotripsy AND (gallstone OR cholelithiasis)

magnetic resonance OR MRCP AND (gallstone OR cholelithiasis) percutaneous AND (gallstone OR cholelithiasis)

sphincteroplasty OR endoscopic balloon dilation AND (gallstone OR cholelithiasis)

sphincterotomy AND (gallstone OR cholelithiasis)

ursodeoxycholic acid AND (gallstone OR cholelithiasis)

\section{Addendum D: Mitglieder der Arbeitsgruppen \\ $\nabla$}

Arbeitsgruppe Prävention und konservative Therapie der Cholelithiasis

F. Lammert, G. Paumgartner (Vorsitzende), D. Klass, H.-J. Krämling, R. Kubitz, C. Pagenstecher, G. Sauter, A. Sommer, H. Wittenburg

\section{Arbeitsgruppe Diagnostik der Cholezysto- und Choledocho-} lithiasis

L. Greiner, K. Ludwig (Vorsitzende), F.-L. Dumoulin, M. Gebel, S. Rickes, J. Textor

Arbeitsgruppe Chirurgische Therapie der Cholezystolithiasis H. Feussner, T. Sauerbruch (Vorsitzende), N. Busch, U. Beuers, A. Kautz (Patientenvertreter), V. Lange, V. Paolucci, B. Schneider

\section{Arbeitsgruppen Therapie der Choledocholithiasis und biliäre} Pankreatitis

F. Hagenmüller, H. Neuhaus, M. W. Neubrand, K.-H. Kiehne (Vorsitzende), Th.P. Hüttl, K. Jessen, S.Jonas, D. Jüngst, V. Keim, M. Maier, G. Otto, H. Schild, M. Schepke, F. Schreiber, H.-J. Schulz, H. Seifert, I. van Thiel (Patientenvertreter), M. Trauner

\section{Arbeitsgruppe Qualitätssicherung}

R. Bittner, J. F. Riemann (Vorsitzende), H. Barlag, H. Bauer, W. O. Bechstein, P. Büchler, P. Frühmorgen, I. Gockel, R. Jakobs, A. Hirner, E. Kraas, J. Maiss, M. Sackmann 


\begin{tabular}{|c|c|c|c|}
\hline Name & Ort & Fachgesellschaft/Organisation ${ }^{1}$ & Finanzielle Interessenkonflikte ${ }^{2}$ \\
\hline Barlag, $\mathrm{H}$. & Düsseldorf & BQS & - \\
\hline Bauer, $\mathrm{H}$. & Berlin & DGCH, BQS, DKG & - \\
\hline Bechstein, W. O. & Frankfurt a. M. & DGVC, GASL & - \\
\hline Beuers, U. & München & DGVS, GASL & - \\
\hline Bittner, R. & Stuttgart & DGVC & - \\
\hline Busch, N. & Würselen & DGVS & - \\
\hline Büchler, P. & Heidelberg & DGVC & - \\
\hline Dumoulin, F.-L. & Bocholt & DGVS, GASL & - \\
\hline Feussner, $\mathrm{H}$. & München & DGVC & - \\
\hline Frühmorgen, $\mathrm{P}$. & Ludwigsburg & DGVS & - \\
\hline Gebel, M. & Hannover & DGVS, DEGUM, GASL & - \\
\hline Gockel, I. & Mainz & DGVC & - \\
\hline Greiner, L. & Wuppertal & DGVS, ALGK, DEGUM & - \\
\hline Hagenmüller, F. & Hamburg & DGVS & - \\
\hline Hirner, A. & Bonn & DGVC, GASL & - \\
\hline Hüttl, Th.P. & München & DGVC & - \\
\hline Jakobs, R. & Wetzlar & DGVS, ALGK & - \\
\hline Jessen, $\mathrm{K}$. & Kiel & DGVS & - \\
\hline Jonas, S. & Berlin & DGVC, GASL & - \\
\hline Jüngst, D. & München & DGVS & - \\
\hline Kautz, A. & Köln & $\mathrm{DL}$ & - \\
\hline Keim, V. & Leipzig & DGVS & - \\
\hline Kiehne, K.-H. & Kiel & DGVS & - \\
\hline Klass, D. & Ulm & DGVS, GASL & - \\
\hline Kraas, E. & Berlin & DGVC, BQS & - \\
\hline Krämling, H.-J. & Düsseldorf & DGVC & - \\
\hline Kubitz, R. & Düsseldorf & DGVS, GASL & - \\
\hline Lammert, F. & Bonn & DGVS, GASL & - \\
\hline Lange, V. & Berlin & DGVC & - \\
\hline Ludwig, K. & Rostock & DGVC & - \\
\hline Maier, M. & Püttlingen & DGVS, ALGK & - \\
\hline Maiß, J. & Erlangen & DGVS & - \\
\hline Neubrand, M. W. & Remagen & DGVS & - \\
\hline Neuhaus, $\mathrm{H}$. & Düsseldorf & DGVS & - \\
\hline Otto, G. & Mainz & DGVC, GASL & - \\
\hline Pagenstecher, C. & Bonn & GFH & - \\
\hline Paolucci, V. & Offenbach & DGVC & - \\
\hline Paumgartner, G. & München & DGVS, GASL & - \\
\hline Rickes, S. & Halberstadt & DGVS, DEGUM & - \\
\hline Riemann, J. F. & Ludwigshafen & DGVS, ALGK & - \\
\hline Sackmann, M. & Bamberg & DGVS & - \\
\hline Sauerbruch, T. & Bonn & DGVS, GASL & - \\
\hline Sauter, G. & München & DGVS & - \\
\hline Schepke, M. & Bonn & DGVS, GASL & - \\
\hline Schild, H. H. & Bonn & DRG & - \\
\hline Schneider, B. & Bonn & DGVC & - \\
\hline Schreiber, F. & Graz & ÖGGH & - \\
\hline Schulz, H.-J. & Berlin & DGVS & - \\
\hline Seifert, H. & Oldenburg & DGVS & - \\
\hline Sommer, A. & Köln & DGVS & - \\
\hline Textor, J. & Bonn & DRG & - \\
\hline Trauner, M. & Graz & ÖGGH & - \\
\hline van Thiel, I. & Köln & $\mathrm{DL}$ & - \\
\hline Werner, J. & Heidelberg & DGVC & - \\
\hline Wittenburg, H. & Leipzig & DGVS, GASL & - \\
\hline
\end{tabular}

Tab. 12 Teilnehmer

1 ALGK, Arbeitsgemeinschaft leitender gastroenterologischer Krankenhausärzte; BQS, Bundesgeschäftsstelle Qualitätssicherung, Fachgruppe Viszeralchirurgie; DEGUM, Deutsche Gesellschaft für Ultraschall in der Medizin; DGCH, Deutsche Gesellschaft für Chirurgie; DGVC, Deutsche Gesellschaft für Viszeralchirurgie; DGVS, Deutsche Gesellschaft für Verdauungs- und Stoffwechselkrankheiten; DKG, Deutsche Krankenhausgesellschaft; DL, Deutsche Leberhilfe; DRG, Deutsche Röntgengesellschaft; GASL, Deutsche Arbeitsgemeinschaft zum Studium der Leber; GFH, Deutsche Gesellschaft für Humangenetik; ÖGGH, Österreichische Gesellschaft für Gastroenterologie und Hepatologie.

2 Siehe I. Methodisches Vorgehen (Leitlinienreport). 


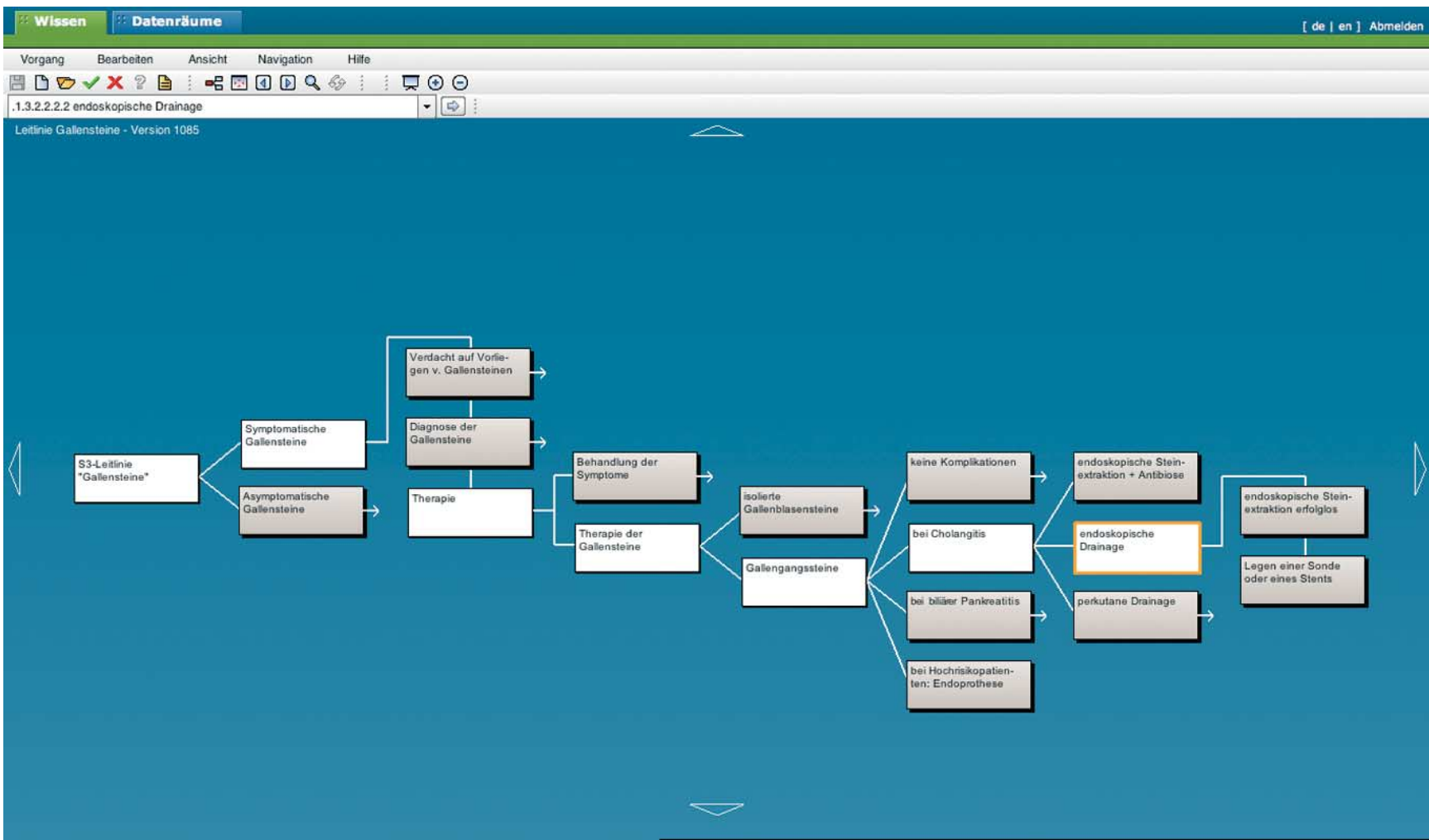

Abb. 1 Klinischer Entscheidungsbaum (Ausschnitt) zur Diagnose und Therapie von Gallensteinen (symptomatische und asymptomatische Gallenblasenund/oder Gallengangssteine). Die Baumstruktur wurde mithilfe der Methode knowledgeTools ${ }^{\circledR}$ entwickelt (http://www.knowledgetools.de/).

\section{Affiliations}

Medizinische Klinik und Poliklinik I, Universitätsklinikum Bonn Innere Abteilung, Krankenhaus Maria Stern Remagen

Klinik für Allgemein- und Viszeralchirurgie, Marienhospital Stuttgart

${ }^{4}$ Chirurgische Universitätsklinik, Klinikum rechts der Isar der Technischen Universität München

Medizinische Klinik 2, Helios Klinikum Wupperta

1. Medizinische Abteilung - Gastroenterologie, Asklepios Klinik Altona

I. Medizinische Klinik, Klinik für Allgemeine Innere Medizin, Universitätsklinikum Schleswig-Holstein, Campus Kiel

8 Klinik für Chirurgie, Klinikum Südstadt Rostock

9 Medizinische Klinik, Evangelisches Krankenhaus Düsseldorf

10 Klinikum Großhadern der Ludwig-Maximilians-Universität München

1 Medizinische Klinik C, Klinikum der Stadt Ludwigshafen am Rhein

\section{Literatur}

1 Berndt H, Nürnberg D, Pannwitz H. Prävalenz der Cholelithiasis. Ergebnisse einer epidemiologischen Studie mittels Sonografie in der DDR. Z Gastroenterol 1989; 27: 662-666

2 Kratzer W, Mason RA, Kachele V. Prevalence of gallstones in sonographic surveys worldwide. J Clin Ultrasound 1999; 27: 1-7

3 Lammert F, Sauerbruch T. Mechanisms of disease: the genetic epidemiology of gallbladder stones. Nat Clin Pract Gastroenterol Hepatol 2005; 2: 423-433

4 Völzke H, Baumeister SE, Alte D et al. Independent risk factors for gallstone formation in a region with high cholelithiasis prevalence. Digestion 2005; 71: 97-105

5 Statistisches Bundesamt. Fallpauschalenbezogene Krankenhausstatistik (DRG-Statistik), Diagnosen und Prozeduren der vollstationären Patienten und Patientinnen in Krankenhäusern 2005. Fachserie 12, Reihe 6.4. Wiesbaden: Statistisches Bundesamt, 2007

6 Sandler RS, Everhart JE, Donowitz $M$ et al. The burden of selected digestive diseases in the United States. Gastroenterology 2002; 122: $1500-1511$

7 Neubrand M, Sackmann M, Caspary W et al. Leitlinien der Deutschen Gesellschaft für Verdauungs- und Stoffwechselkrankheiten zur Behandlung von Gallensteinen. Z Gastroenterol 2000; 38: 449-468
8 Lorenz W, Ollenschläger G, Geraedts M et al. Das Leitlinien-Manual. Entwicklung und Implementierung von Leitlinien in der Medizin. Z Ärztl Fortbild Qual Gesundheitswes 2001; 95 (Suppl I): 1-84

9 Beyer M, Geraedts M, Gerlach FM et al. Deutsches Instrument zur methodischen Leitlinien-Bewertung (DELBI). Z Ärztl Fortbild Qual Gesundheitswes 2006; 99: 468-492

10 Hoffmann JC, Fischer I, Hohne W et al. Methodische Basis für die Entwicklung von Konsensusempfehlungen. Z Gastroenterol 2004; 42: 984-986

11 Ollenschläger G, Kirchner $H$, Berenbeck $C$ et al. Aktuelle Initiativen zur Realisierung nationaler Leitlinien in Deutschland - eine Übersicht. Gesundheitswesen 2002; 64: 513-520

12 Kirchner H, Fiene M, Ollenschläger G. Bewertung und Implementierung von Leitlinien. Rehabilitation 2003; 42: 74-82

13 Breidenbach S. Transparenz von Recht durch knowledgeTools. Versicherungswissenschaftliche Studien 2003; 24: 131-136

14 Leese T, Neoptolemos JP, Baker AR et al. Management of acute cholangitis and the impact of endoscopic sphincterotomy. Br J Surg 1986; 73: 988-992

15 Lai EC, Mok FP, Tan ES et al. Endoscopic biliary drainage for severe acute cholangitis. N Engl J Med 1992; 326: 1582-1586

16 Leung JW, Ling TK, Chan RC et al. Antibiotics, biliary sepsis, and bile duct stones. Gastrointest Endosc 1994; 40: 716-721

17 Sharma BC, Agarwal DK, Baijal SS et al. Endoscopic management of acute calculous cholangitis. J Gastroenterol Hepatol 1997; 12: 874-876

18 Shea JA, Berlin JA, Escarce JJ et al. Revised estimates of diagnostic test sensitivity and specificity in suspected biliary tract disease. Arch Intern Med 1994; 154: 2573-2581

19 Trowbridge RL, Rutkowski NK, Shojania KG. Does this patient have acute cholecystitis? JAMA 2003; 289: 80-86

20 Tenner S, Dubner H, Steinberg W. Predicting gallstone pancreatitis with laboratory parameters: a meta-analysis. Am J Gastroenterol 1994; 89: 1863-1866

21 Ammori BJ, Boreham B, Lewis P et al. The biochemical detection of biliary etiology of acute pancreatitis on admission: a revisit in the modern era of biliary imaging. Pancreas 2003; 26: e32-e35

22 Lévy P, Boruchowicz A, Hastier P et al. Diagnostic criteria in predicting a biliary origin of acute pancreatitis in the era of endoscopic ultra- 
sound: multicentre prospective evaluation of 213 patients. Pancreatology 2005; 5: 450-456

23 Liu CL, Fan ST, Lo CM et al. Clinico-biochemical prediction of biliary cause of acute pancreatitis in the era of endoscopic ultrasonography. Aliment Pharmacol Ther 2005; 22: 423-431

24 Sackmann M. Behandlung der biliären Kolik. Dtsch Med Wochenschr 1998; 123: 945-947

25 Festi D, Sottili S, Colecchia A et al. Clinical manifestations of gallstone disease: evidence from the multicenter Italian study on cholelithiasis (MICOL). Hepatology 1999; 30: 839-846

26 Rünzi M, Layer P, Büchler MW et al. Therapie der akuten Pankreatitis. Gemeinsame Leitlinien. Z Gastroenterol 2000; 38: 571-581

27 Berger MY, van der Velden JJ, Lijmer JG et al. Abdominal symptoms: do they predict gallstones? A systematic review. Scand J Gastroenterol 2000; 35: 70-76

28 Jørgensen T. Abdominal symptoms and gallstone disease: an epidemiological investigation. Hepatology 1989; 9: 856-860

29 Berhane T, Vetrhus M, Hausken T et al. Pain attacks in non-complicated and complicated gallstone disease have a characteristic pattern and are accompanied by dyspepsia in most patients: the results of a prospective study. Scand J Gastroenterol 2006; 41: 93-101

30 Glambek I, Arnesjo B, Soreide O. Correlation between gallstones and abdominal symptoms in a random population. Results from a screening study. Scand J Gastroenterol 1989; 24: 277-281

31 Kennedy TM, Jones RH. Epidemiology of cholecystectomy and irritable bowel syndrome in a UK population. Br J Surg 2000; 87: 1658-1663

32 Weinert CR, Arnett D, Jacobs D et al. Relationship between persistence of abdominal symptomes and successful outcome after cholecystectomy. Arch Intern Med 2000; 160: 989-995

33 Thistle JL, Cleary PA, Lachin JM et al. The natural history of cholelithiasis: the National Cooperative Gallstone Study. Ann Intern Med 1984; 101: 171-175

34 Friedman GD, Raviola CA, Fireman B. Prognosis of gallstones with mild or no symptoms: 25 years of follow-up in a health maintenance organization. J Clin Epidemiol 1989; 42: 127-136

35 Ransohoff DF, Gracie WA. Treatment of gallstones. Ann Intern Med 1993; 119: 606-619

36 Attili AF, De Santis A, Capri $R$ et al. The natural history of gallstones: the GREPCO experience. Hepatology 1995; 21: 655-660

37 Gebel M. Die Sonografie beim Gallensteinleiden. Chir Gastroenterol 1989; 5: 469-476

38 Macheiner P, Gritzmann N. Sonografie der Streßcholezystitis - Diagnose und Differentialdiagnose. Z Gastroenterol 1996; 34: 21-26

39 Ralls PW, Colletti PM, Lapin SA et al. Real-time sonography in suspected acute cholecystitis. Prospective evaluation of primary and secondary signs. Radiology 1985; 155: 767-771

40 Angelico M, Gandin C, Canuzzi P et al. Gallstones in cystic fibrosis: a critical reappraisal. Hepatology 1991; 14: 768-775

41 Duggirala R, Mitchell BD, Blangero J et al. Genetic determinants of variation in gallbladder disease in the Mexican-American population. Genet Epidemiol 1999; 16: 191-204

42 Nakeeb A, Comuzzie AG, Martin L et al. Gallstones: genetics versus environment. Ann Surg 2002; 235: 842-849

43 Katsika D, Grjibovski A, Einarsson C et al. Genetic and environmental influences on symptomatic gallstone disease: a Swedish study of 43,141 twin pairs. Hepatology 2005; 41: 1138-1143

44 Puppala S, Dodd GD, Fowler $S$ et al. A genomewide search finds major susceptibility loci for gallbladder disease on chromosome 1 in Mexican Americans. Am J Hum Genet 2006; 78: 377-392

45 Rosmorduc 0 , Hermelin B, Poupon R. MDR3 gene defect in adults with symptomatic intrahepatic and gallbladder cholesterol cholelithiasis. Gastroenterology 2001; 120: 1459-1467

46 Rosmorduc $O$, Hermelin B, Boelle PY et al. ABCB4 gene mutation-associated cholelithiasis in adults. Gastroenterology 2003; 125: 452-459

47 Fracchia $M$, Pellegrino $S$, Secreto $P$ et al. Biliary lipid composition in cholesterol microlithiasis. Gut 2001; 48: 702-706

48 Buch S, Schafmayer C, Völzke H et al. A genome-wide association scan identifies the hepatic cholesterol transporter ABCG8 as a susceptibility factor for human gallstone disease. Nat Genet 2007; 46: 995-999

49 Grünhage F, Acalovschi M, Tirziu S et al. Increased gallstone risk in humans conferred by common variant of hepatic ATP binding cassette transporter for cholesterol. Hepatology 2007; 46: epub
50 Van Mil SW, van der Woerd WL, van der Brugge G et al. Benign recurrent intrahepatic cholestasis type 2 is caused by mutations in ABCB11. Gastroenterology 2004; 127: 379-384

51 Venneman NG, Besselink MG, Keulemans YC et al. Ursodeoxycholic acid exerts no beneficial effect in patients with symptomatic gallstones awaiting cholecystectomy. Hepatology 2006; 43: 1276-1283

52 McSherry CK, Ferstenberg H, Calhoun WF et al. The natural history of diagnosed gallstone disease in symptomatic and asymptomatic patients. Ann Surg 1985; 202: 59-63

53 Ransohoff DF, Gracie WA. Management of patients with symptomatic gallstones: a quantitative analysis. Am J Med 1990; 88: 154-160

54 Bates T, Ebbs SR, Harrison $M$ et al. Influence of cholecystectomy on symptoms. Br J Surg 1991; 78: 964-967

55 Qureshi MA, Burke PE, Brindley NM et al. Post-cholecystectomy symptoms after laparoscopic cholecystectomy. Ann R Coll Surg Engl 1993; 75: 349-353

56 Plaisier PW, van der Hul RL, Nijs HG et al. The course of biliary and gastrointestinal symptoms after treatment of uncomplicated symptomatic gallstones: results of a randomized study comparing extracorporeal shock wave lithotripsy with conventional cholecystectomy. Am J Gastroenterol 1994; 89: 739-744

57 Lublin M, Crawford DL, Hiatt JR et al. Symptoms before and after laparoscopic cholecystectomy for gallstones. Am Surg 2004; 70: 863-866

58 Vetrhus $M$, Berhane $T$, Soreide 0 et al. Pain persists in many patients five years after removal of the gallbladder: observations from two randomized controlled trials of symptomatic, noncomplicated gallstone disease and acute cholecystitis. J Gastrointest Surg 2005; 9: 826-831

59 Gracie WA, Ransohoff DF. The natural history of silent gallstones: the innocent gallstone is not a myth. N Engl J Med 1982; 307: 798-800

60 Friedman GD. Natural history of asymptomatic and symptomatic gallstones. Am J Surg 1993; 165: 399-404

61 Ransohoff DF, Gracie WA, Wolfenson LB et al. Prophylactic cholecystectomy or expectant management for silent gallstones. A decision analysis to assess survival. Ann Intern Med 1983; 99: 199-204

62 Sonnenberg A, Derfus GA, Soergel KH. Lithotripsy versus cholecystectomy for management of gallstones. A decision analysis by Markov process. Dig Dis Sci 1991; 36: 949-956

63 Randi G, Franceschi S, LaVecchia C. Gallbladder cancer worldwide: geographical distribution and risk factors. Int J Cancer 2006; 118: 1591-1602

64 Maringhini A, Moreau JA, Melton LJ et al. Gallstones, gallbladder cancer, and other gastrointestinal malignancies. An epidemiologic study in Rochester, Minnesota. Ann Intern Med 1987; 107: 30-35

65 Sheth S, Bedford A, Chopra S. Primary gallbladder cancer: recognition of risk factors and the role of prophylactic cholecystectomy. Am J Gastroenterol 2000; 95: 1402-1410

66 Aucott JN, Cooper GS, Bloom AD et al. Management of gallstones in diabetic patients. Arch Intern Med 1993; 153: 1053-1058

67 Law CHL, McKay D, Tandan VR. Gallstone disease. In: McDonald JWD, Burroughs A, Feagan BG (Hrsg). Evidence-based Gastroenterology and Child Health. Oxford: Blackwell Publishing, 2004: 311-320

68 Ashur H, Siegal B, Oland Y et al. Calcified gallbladder (porcelain gallbladder). Arch Surg 1978; 113: 594-596

69 Towfigh S, McFadden DW, Cortina GR et al. Porcelain gallbladder is not associated with gallbladder carcinoma. Am Surg 2001; 67: 7-10

70 Stephen AE, Berger DL. Carcinoma in the porcelain gallbladder: a relationship revisited. Surgery 2001; 129: 699-703

71 Diehl AK. Gallstone size and the risk of gallbladder cancer. JAMA 1983; 250: 2323-2326

72 Lowenfels $A B$, Walker AM, Althaus DP et al. Gallstone growth, size, and risk of gallbladder cancer: an interracial study. Int J Epidemiol 1989; 18: $50-54$

73 Lowenfels $A B$, Lindstrom CG, Conway MJ et al. Gallstones and risk of gallbladder cancer. J Natl Cancer Inst 1985; 75: 77-80

74 Jørgensen $T$, Jensen $K H$. Polyps in the gallbladder. A prevalence study. Scand J Gastroenterol 1990; 25: 281-286

75 Chen CY, Lu CL, Chang FY et al. Risk factors for gallbladder polyps in the Chinese population. Am J Gastroenterol 1997; 92: 2066-2068

76 Okamoto $M$, Okamoto $H$, Kitahara $F$ et al. Ultrasonographic evidence of association of polyps and stones with gallbladder cancer. Am J Gastroenterol 1999; 94: 446-450 
77 Myers RP, Shaffer EA, Beck PL. Gallbladder polyps: epidemiology, natural history and management. Can J Gastroenterol 2002; 16: 187-194

78 Heyder N, Gunter E, Giedl J et al. Polypoide Läsionen der Gallenblase. Dtsch Med Wochenschr 1990; 115: 243-247

79 Persley KM. Gallbladder polyps. Curr Treat Options Gastroenterol 2005; 8: 105-108

80 Yang HL, Sun YG, Wang Z. Polypoid lesions of the gallbladder: diagnosis and indications for surgery. Br J Surg 1992; 79: 227-229

81 Mainprize KS, Gould SW, Gilbert JM. Surgical management of polypoid lesions of the gallbladder. Br J Surg 2000; 87: 414-417

82 Terzi C, Sokmen S, Seckin S et al. Polypoid lesions of the gallbladder: report of 100 cases with special reference to operative indications. Surgery 2000; 127: 622-627

83 Lee KF, Wong J, Li JC et al. Polypoid lesions of the gallbladder. Am J Surg 2004; 188: 186-190

84 Moriguchi H, Tazawa J, Hayashi Y et al. Natural history of polypoid lesions in the gall bladder. Gut 1996; 39: 860-862

85 Csendes A, Burgos AM, Csendes P et al. Late follow-up of polypoid lesions of the gallbladder smaller than $10 \mathrm{~mm}$. Ann Surg 2001; 234: 657-660

86 Koga A, Watanabe H, Fukuyama T. Diagnosis and operative indication for polypoid lesions of the gallbladder. Arch Surg 1988; 123: 26-29

87 Kubota K, Bandai Y, Noie T et al. How should polypoid lesions of the gallbladder be treated in the era of laparoscopic cholecystectomy? Surgery 1995; 117: 481-487

88 Nürnberg D, Ignee A, Dietrich CF. Aktueller Stand der Sonografie in der Gastroenterologie. Biliopankreatisches System. Med Klinik 2007 102: $112-126$

89 Azuma T, Yoshikawa T, Araida T et al. Differential diagnosis of polypoid lesions of the gallbladder by endoscopic ultrasonography. Am J Surg 2001; 181: 65-70

90 Sugiyama M, Atomi Y, Yamato T. Endoscopic ultrasonography for differential diagnosis of polypoid gall bladder lesions: analysis in surgical and follow up series. Gut 2000; 46: 250-254

91 O'Brien PE, Dixon JB. A rational approach to cholelithiasis in bariatric surgery: its application to the laparoscopically placed adjustable gastric band. Arch Surg 2003; 138: 908-912

92 Swartz DE, Felix EL. Elective cholecystectomy after Roux-en-Y gastric bypass: why should asymptomatic gallstones be treated differently in morbidly obese patients? Surg Obes Relat Dis 2005; 1: 555-560

93 Lapidus A, Bangstad M, Astrom $M$ et al. The prevalence of gallstone disease in a defined cohort of patients with Crohn's disease. Am J Gastroenterol 1999; 94: 1261-1266

94 Fraquelli M, Losco A, Visentin S et al. Gallstone disease and related risk factors in patients with Crohn disease: analysis of 330 consecutive cases. Arch Intern Med 2001; 161: 2201-2204

95 Lindner $H$, Bauer $H$. Untersuchungen zur Gallenblasenmotilität nach totaler Gastrektomie. Viszeralchirurgie 2002; 327: 299-304

96 Kobayashi T, Hisanaga M, Kanehiro $\mathrm{H}$ et al. Analysis of risk factors for the development of gallstones after gastrectomy. Br J Surg 2005; 92: 1399-1403

97 Chew SS, Ngo TQ Douglas PR et al. Cholecystectomy in patients with Crohn's ileitis. Dis Colon Rectum 2003; 46: 1484-1488

98 Thompson JS. The role of prophylactic cholecystectomy in the shortbowel syndrome. Arch Surg 1996; 131: 556-559

99 Greenstein SM, Katz S, Sun S et al. Prevalence of asymptomatic cholelithiasis and risk of acute cholecystitis after kidney transplantation. Transplantation 1997; 63: 1030-1032

100 Melvin WS, Meier DJ, Elkhammas EA et al. Prophylactic cholecystectomy is not indicated following renal transplantation. Am J Surg 1998; 175: $317-319$

101 Jackson T, Treleaven D, Arlen D et al. Management of asymptomatic cholelithiasis for patients awaiting renal transplantation. Surg Endosc 2005; 19: 510-513

102 Kao LS, Flowers C, Flum DR. Prophylactic cholecystectomy in transplant patients: a decision analysis. J Gastrointest Surg 2005; 9: 965-972

103 Milas M, Ricketts RR, Amerson JR et al. Management of biliary tract stones in heart transplant patients. Ann Surg 1996; 223: 747-753

104 Hüttl TP, Hrdina C, Krämling HJ et al. Gallstone surgery in German university hospitals. Development, complications and changing strategies. Langenbecks Arch Surg 2001; 386: 410-417
105 Gleisberg C, Seidel JH, Bauer H et al. Cholezystektomie In: Bundesgeschäftsstelle Qualitätssicherung (Hrsg). Qualität sichtbar machen BQS-Qualitätsbericht 2001. Düsseldorf, 2002: 47-53

106 Ludwig K, Köckerling F, Hohenberger $W$ et al. Die chirurgische Therapie der Cholecysto-/Choledocholithiasis. Ergebnisse einer deutschlandweiten Umfrage an 859 Kliniken mit 123090 Cholecystektomien. Chirurg 2001; 72: 1171-1178

107 Velanovich V, Morton JM, McDonald $M$ et al. Analysis of the SAGES outcomes initiative cholecystectomy registry. Surg Endosc 2006; 20: $43-50$

108 Keus F, de Jong JA, Gooszen HG et al. Laparoscopic versus open cholecystectomy for patients with symptomatic cholecystolithiasis. Cochrane Database Syst Rev 2006; CD006231

109 Putensen-Himmer G, Putensen C, Lammer H et al. Comparison of postoperative respiratory function after laparoscopy or open laparotomy for cholecystectomy. Anesthesiology 1992; 77: 675-680

110 Agnifili A, Verzaro R, Colangeli $A$ et al. Perioperative pulmonaryfunction, pain and stress-response after cholecystectomy performed via laparotomy or laparoscopy - comparison between laparoscopy and laparotomy. Minim Invasive Ther Allied Technol 1993; 2: 283-288

111 Coelho JC, de Araujo RP, Marchesini JB et al. Pulmonary function after cholecystectomy performed through Kocher's incision, a mini-incision, and laparoscopy. World J Surg 1993; 17: 544-546

112 Garcia-Caballero M, Vara-Thorbeck $C$. The evolution of postoperative ileus after laparoscopic cholecystectomy. A comparative study with conventional cholecystectomy and sympathetic blockade treatment. Surg Endosc 1993; 7: 416-419

113 Trondsen E, Reiertsen O, Andersen OK et al. Laparoscopic and open cholecystectomy. A prospective, randomized study. Eur J Surg 1993; 159: 217-221

114 Berggren U, Gordh T, Grama D et al. Laparoscopic versus open cholecystectomy: hospitalization, sick leave, analgesia and trauma responses. Br J Surg 1994; 81: 1362-1365

115 Dionigi R, Dominioni L, Benevento A et al. Effects of surgical trauma of laparoscopic vs. open cholecystectomy. Hepatogastroenterology 1994; 41: 471-476

116 Kjaersgaard P, Reiertsen O, Trondsen $E$ et al. Comparison of sequential and fixed-sample designs in a controlled clinical trial with laparoscopic versus conventional cholecystectomy. Scand J Gastroenterol 1994; 29: 854-858

117 Milheiro A, Sousa FC, Manso EC et al. Metabolic responses to cholecystectomy: open vs. laparoscopic approach. J Laparoendosc Surg 1994; 4: $311-317$

118 Dauleh MI, Rahman S, Townell NH. Open versus laparoscopic cholecystectomy: a comparison of postoperative temperature. J R Coll Surg Edinb 1995; 40: 116-118

119 Essen P, Thorell A, McNurlan MA et al. Laparoscopic cholecystectomy does not prevent the postoperative protein catabolic response in muscle. Ann Surg 1995; 222: 36-42

120 Huang SM, Wu CW, Lui WY et al. A prospective randomised study of laparoscopic vs. open cholecystectomy in aged patients with cholecystolithiasis. S Afr J Surg 1996; 34: 177-179

121 Koprulu G, Esen F, Pembeci Ket al. Pulmonary mechanics during laparoscopic surgery. Adv Exp Med Biol 1996; 388: 643-646

122 Ortega $A E$, Peters $J H$, Incarbone $R$ et al. A prospective randomized comparison of the metabolic and stress hormonal responses of laparoscopic and open cholecystectomy. J Am Coll Surg 1996; 183: $249-256$

123 Rovina N, Bouros D, Tzanakis $N$ et al. Effects of laparoscopic cholecystectomy on global respiratory muscle strength. Am J Respir Crit Care Med 1996; 153: 458-461

$124 \mathrm{Gal}$ I, Roth E, Lantos J et al. Inflammatory mediators and surgical trauma regarding laparoscopic access: free radical mediated reactions. Acta Chir Hung 1997; 36: 97-99

125 Karayiannakis AJ, Makri GG, Mantzioka A et al. Systemic stress response after laparoscopic or open cholecystectomy: a randomized trial. Br J Surg 1997; 84: 467-471

126 Bellon JM, Manzano L, Larrad A et al. Endocrine and immune response to injury after open and laparoscopic cholecystectomy. Int Surg 1998; 83: 24-27

127 Chumillas MS, Ponce JL, Delgado F et al. Pulmonary function and complications after laparoscopic cholecystectomy. Eur J Surg 1998; 164 : 433-437 
128 Engin A, Bozkurt BS, Ersoy E et al. Stress hyperglycemia in minimally invasive surgery. Surg Laparosc Endosc 1998; 8: 435-437

129 Lujan JA, Sanchez-Bueno F, Parrilla P et al. Laparoscopic vs. open cholecystectomy in patients aged 65 and older. Surg Laparosc Endosc 1998; 8: 208-210

130 Volpino $P$, Cangemi V, D'Andrea $N$ et al. Hemodynamic and pulmonary changes during and after laparoscopic cholecystectomy. A comparison with traditional surgery. Surg Endosc 1998; 12: 119-123

131 Chaudhary D, Verma GR, Gupta R et al. Comparative evaluation of the inflammatory mediators in patients undergoing laparoscopic versus conventional cholecystectomy. Aust N Z J Surg 1999; 69: 369-372

132 Lausten SB, Ibrahim TM, El-Sefi T et al. Systemic and cell-mediated immune response after laparoscopic and open cholecystectomy in patients with chronic liver disease. A randomized, prospective study. Dig Surg 1999; 16: 471-477

133 LeBlanc-Louvry I, Coquerel A, Koning E et al. Operative stress response is reduced after laparoscopic compared to open cholecystectomy: the relationship with postoperative pain and ileus. Dig Dis Sci 2000; 45: $1703-1713$

134 Coskun I, Hatipoglu AR, Topaloglu A et al. Laparoscopic versus open cholecystectomy: effect on pulmonary function tests. Hepatogastroenterology 2000; 47: 341-342

135 Demirer S, Karadayi K, Simsek $S$ et al. Comparison of postoperative acute-phase reactants in patients who underwent laparoscopic vs open cholecystectomy: a randomized study. J Laparoendosc Adv Surg Tech A 2000; 10: 249-252

136 Hendolin HI, Paakonen ME, Alhava EM et al. Laparoscopic or open cholecystectomy: a prospective randomised trial to compare postoperative pain, pulmonary function, and stress response. Eur J Surg 2000; 166: 394-399

137 Mimica Z, Biocic M, Bacic A et al. Laparoscopic and laparotomic cholecystectomy: a randomized trial comparing postoperative respiratory function. Respiration 2000; 67: 153-158

138 Prisco D, De Gaudio AR, Carla $R$ et al. Videolaparoscopic cholecystectomy induces a hemostasis activation of lower grade than does open surgery. Surg Endosc 2000; 14: 170-174

139 Galizia G, Prizio G, Lieto E et al. Hemodynamic and pulmonary changes during open, carbon dioxide pneumoperitoneum and abdominal wall-lifting cholecystectomy. A prospective, randomized study. Surg Endosc 2001; 15: 477-483

140 Hasukic S, Mesic D, Dizdarevic E et al. Pulmonary function after laparoscopic and open cholecystectomy. Surg Endosc 2002; 16: 163-165

141 Zulfikaroglu B, Koc M, Soran A et al. Evaluation of oxidative stress in laparoscopic cholecystectomy. Surg Today 2002; 32: 869-874

142 Luo K, Li JS, Li LT et al. Operative stress response and energy metabolism after laparoscopic cholecystectomy compared to open surgery. World J Gastroenterol 2003; 9: 847-850

143 Bukan MH, Bukan N, Kaymakcioglu N et al. Effects of open vs. laparoscopic cholecystectomy on oxidative stress. Tohoku J Exp Med 2004; 202: 51-56

144 Bosch F, Wehrman U, Saeger HD et al. Laparoscopic or open conventional cholecystectomy: clinical and economic considerations. Eur J Surg 2002; 168: 270-277

145 Shea JA, Healey MJ, Berlin JA et al. Mortality and complications associated with laparoscopic cholectystectomy. A meta-analysis. Ann Surg 1996; 224: 609-620

146 Ludwig K, Bernhardt B, Wilhelm L et al. Gallenwegsverletzungen bei laparoskopischer Cholezystektomie. Viszeralchirurgie 2002; 37: 61-66

147 Bundesgeschäftsstelle Qualitätssicherung. BQS-Bundesauswertung 2005 Cholezystektomie. Düsseldorf, 2006: 1-26

148 Schiedeck TH, Schulte T, Gunarsson R et al. Laparoskopische Cholezystektomie bei akuter Cholezystitis. Minimal Invasive Chirurgie 1997; 6: 48-51

149 Kiviluoto T, Siren J, Luukkonen P et al. Randomised trial of laparoscopic versus open cholecystectomy for acute and gangrenous cholecystitis. Lancet 1998; 31: 321-325

150 Johansson M, Thune A, Nelvin L et al. Randomized clinical trial of open versus laparoscopic cholecystectomy in the treatment of acute cholecystitis. Br J Surg 2005; 92: 44-49

151 Puggioni A, Wong LL. A metaanalysis of laparoscopic cholecystectomy in patients with cirrhosis. J Am Coll Surg 2003; 197: 921-926

152 Currò G, Iapichino G, Melita G et al. Laparoscopic cholecystectomy in Child-Pugh class C cirrhotic patients. JSLS 2005; 9: 311-315
153 Perkins L, Jeffries M, Patel T. Utility of preoperative scores for predicting morbidity after cholecystectomy in patients with cirrhosis. Clin Gastroenterol Hepatol 2004; 2: 1123-1128

154 Desai DC, Smink RD. Mirizzi syndrome type II: is laparoscopic cholecystectomy justified? JSLS 1997; 1: 237-239

155 Yeh CN, Jan YY, Chen MF. Laparoscopic treatment for Mirizzi syndrome. Surg Endosc 2003; 17: 1573-1578

156 Barkun JS, Barkun AN, Sampalis JS et al. Randomised controlled trial of laparoscopic versus mini cholecystectomy. Lancet 1992; 340: 1116-1119

157 Kunz R, Orth K, Vogel J et al. Laparoskopische Cholezystektomie versus Mini-Lap-Cholezystektomie. Chirurg 1992; 63: 291-295

158 Tate JJ, Lau WY, Leung KL et al. Laparoscopic versus mini-incision cholecystectomy. Lancet 1993; 341: 1214-1215

159 McMahon AJ, Russell IT, Baxter JN et al. Laparoscopic versus minilaparotomy cholecystectomy: a randomised trial. Lancet 1994; 343: 135-138

160 Redmond HP, Watson RW, Houghton T et al. Immune function in patients undergoing open vs laparoscopic cholecystectomy. Arch Surg 1994; 129: 1240-1246

161 McGinn FP, Miles AJ, Uglow M et al. Randomized trial of laparoscopic cholecystectomy and mini-cholecystectomy. Br J Surg 1995; 82: 1374-1377

162 Majeed AW, Troy G, Nicholl JP et al. Randomised, prospective, singleblind comparison of laparoscopic versus small-incision cholecystectomy. Lancet 1996; 347: 989-994

163 Bruce DM, Smith M, Walker CB et al. Minimal access surgery for cholelithiasis induces an attenuated acute phase response. Am J Surg 1999; 178: 232-234

164 Ros A, Gustafsson L, Krook H et al. Laparoscopic cholecystectomy versus mini-laparotomy cholecystectomy: a prospective, randomized, single-blind study. Ann Surg 2001; 234: 741-749

165 Srivastava A, Srinivas G, Misra MC et al. Cost-effectiveness analysis of laparoscopic versus minilaparotomy cholecystectomy for gallstone disease. A randomized trial. Int J Technol Assess Health Care 2001; 17: 497-502

166 Grande M, Tucci GF, Adorisio $O$ et al. Systemic acute-phase response after laparoscopic and open cholecystectomy. Surg Endosc 2002; 16: $313-316$

167 Keus F, de Jong JA, Gooszen HG et al. Laparoscopic versus small-incision cholecystectomy for patients with symptomatic cholecystolithiasis. Cochrane Database Syst Rev 2006; CD006229

168 Schwenk W, Neudecker J, Mall J et al. Prospective randomized blinded trial of pulmonary function, pain, and cosmetic results after laparoscopic vs. microlaparoscopic cholecystectomy. Surg Endosc 2000; 14: $345-348$

169 Cheah WK, Lenzi JE, So JB et al. Randomized trial of needlescopic versus laparoscopic cholecystectomy. Br J Surg 2001; 88: 45-47

170 Bisgaard T, Klarskov B, Trap R et al. Microlaparoscopic vs conventional laparoscopic cholecystectomy: a prospective randomized doubleblind trial. Surg Endosc 2002; 16: 458-464

171 Huang MT, Wang W, Wei PL et al. Minilaparoscopic and laparoscopic cholecystectomy: a comparative study. Arch Surg 2003; 138: 1017-1023

172 Hsieh $\mathrm{CH}$. Early minilaparoscopic cholecystectomy in patients with acute cholecystitis. Am J Surg 2003; 185: 344-348

173 Sarli L, Iusco D, Gobbi S et al. Randomized clinical trial of laparoscopic cholecystectomy performed with mini-instruments. Br J Surg 2003; 90: $1345-1348$

174 Linden W, Sunzel $H$. Early versus delayed operation for acute cholecystitis. A controlled clinical trial. Am J Surg 1970; 120: 7-13

175 Lahtinen J, Alhava EM, Aukee S. Acute cholecystitis treated by early and delayed surgery. A controlled clinical trial. Scand J Gastroenterol 1978; 13: 673-678

176 Jarvinen HJ, Hastbacka J. Early cholecystectomy for acute cholecystitis: a prospective randomized study. Ann Surg 1980; 191: 501-505

177 Jaeger G, Rothenbühler JM, Famos M et al. Wann soll die Cholecystektomie bei akuter Cholecystitis vorgenommen werden? Schweiz Med Wschr 1983; 113: 552-554

178 Norrby S, Herlin P, Holmin Tet al. Early or delayed cholecystectomy in acute cholecystitis? A clinical trial. Br J Surg 1983; 70: 163-165

179 Lo CM, Liu CL, Fan ST et al. Prospective randomized study of early versus delayed laparoscopic cholecystectomy for acute cholecystitis. Ann Surg 1998; 227: 461-467 
180 Lai PB, Kwong KH, Leung KL et al. Randomized trial of early versus delayed laporoscopic cholecystectomy for acute cholecystitis. Br J Surg 1998; 85: 764-767

181 Johansson M, Thune A, Blomqvist A et al. Management of acute cholecystitis in the laparoscopic era: results of a prospective, randomized clinical trial. J Gastrointest Surg 2003; 7: 642-645

182 Kolla SB, Aggarwal S, Kumar A et al. Early versus delayed laparoscopic cholecystectomy for acute cholecystitis: a prospective randomized trial. Surg Endosc 2004; 18: 1323-1327

183 Gurusamy KS, Samraj K. Early versus delayed laparoscopic cholecystectomy for acute cholecystitis. Cochrane Database Syst Rev 2006; CD005440

184 Papi C, Catarci M, D’Ambrosio L et al. Timing of cholecystectomy for acute calculous cholecystitis: a meta-analysis. Am J Gastroenterol 2004; 99: 147-155

185 Dietzel M, Lippert H, Gastinger I et al. Die „akute Galle“ - laparoskopische Cholezystektomie häufig möglich. Ergebnisse einer Multizenterstudie der Ostdeutschen Arbeitsgruppe für Leistungserfassung und Qualitätssicherung in der Chirurgie. Zentralbl Chir 2000; 125: 547-551

186 Madan AK, Aliabadi-Wahle S, Tesi D et al. How early is early laparoscopic treatment of acute cholecystitis? Am J Surg 2002; 183: 232-236

187 Hatzidakis AA, Prassopoulos P, Petinarakis I et al. Acute cholecystitis in high-risk patients: percutaneous cholecystostomy vs conservative treatment. Eur Radiol 2002; 12: 1778-1784

188 Vetrhus M, Soreide O, Nesvik I et al. Acute cholecystitis: delayed surgery or observation. A randomized clinical trial. Scand J Gastroenterol 2003; 38: 985-990

189 Ito K, Fujita N, Noda Y et al. Percutaneous cholecystostomy versus gallbladder aspiration for acute cholecystitis: a prospective randomized controlled trial. Am J Roentgenol 2004; 183: 193-196

190 Akyürek N, Salman B, Yuksel O et al. Management of acute calculous cholecystitis in high-risk patients: percutaneous cholecystotomy followed by early laparoscopic cholecystectomy. Surg Laparosc Endosc Percutan Tech 2005; 15: 315-320

191 Schwesinger WH, Sirinek KR, Strodel WE. Laparoscopic cholecystectomy for biliary tract emergencies: state of the art. World J Surg 1999; 23: 334-342

192 Garber SM, Korman J, Cosgrove JM et al. Early laparoscopic cholecystectomy for acute cholecystitis. Surg Endosc 1997; 11: 347-350

193 Wilson RG, Macintyre IM, Nixon SJ et al. Laparoscopic cholecystectomy as a safe and effective treatment for severe acute cholecystitis. $\mathrm{Br}$ Med J 1992; 305: 394-396

194 Bedirli A, Sakrak O, Sozuer EM et al. Factors effecting the complications in the natural history of acute cholecystitis. Hepatogastroenterology 2001; 48: 1275-1278

195 Clavien PA, Richon J, Burgan S et al. Gallstone ileus. Br J Surg 1990; 77: 737-742

196 Misawa T, Koike M, Suzuki Ket al. Ultrasonographic assessment of the risk of injury to branches of the middle hepatic vein during laparoscopic cholecystectomy. Am J Surg 1999; 178: 418-421

197 Kebudi A, Halefoglu AM, Yetkin G et al. Role of preoperative color Doppler ultrasound scan in the evaluation of the risk of injury of major branches of the middle hepatic vein during laparoscopic cholecystectomy. Int Surg 2002; 87: 236-239

198 Shen BY, Li HW, Chen M et al. Color Doppler ultrasonographic assessment of the risk of injury to major branch of the middle hepatic vein during laparoscopic cholecystectomy. Hepatobiliary Pancreat Dis Int 2003; 2: 126-130

199 Yau HM, Lee KT, Kao EL et al. Color Doppler ultrasound detection and classification of the tangential hepatic vein before laparoscopic cholecystectomy. Surg Endosc 2005; 19: 1377-1380

200 Zhang WZ, Shen J, Xie JX et al. Color Doppler ultrasonographic examination on the relationship between the gallbladder bed and major branch of the middle hepatic vein. Hepatobiliary Pancreat Dis Int 2005; 4: 299-301

201 Abboud PA, Malet PF, Berlin JA et al. Predictors of common bile duct stones prior to cholecystectomy: a meta-analysis. Gastrointest Endosc 1996; 44: 450-455

202 Trondsen E, Edwin B, Reiertsen $O$ et al. Prediction of common bile duct stones prior to cholecystectomy: a prospective validation of a discriminant analysis function. Arch Surg 1998; 133: 162-166
203 Ritz J, Germer C, Buhr H. Preoperative routine chest x-ray: expensive and of little value. Langenbecks Arch Chir Suppl Kongressbd 1997; 114: 1051-1053

204 Fahlke J, Ridwelski K, Manger T et al. Diagnostic workup before laparoscopic cholecystectomy - which diagnostic tools should be used? Hepatogastroenterology 2001; 48: 59-65

205 Zehetner J, Shamiyeh A, Wayanad W. Cholecystolithiasis - Update 2006. In: Neugebauer EA, Sauerland S, Fingerhut A, Millat B, Buess G (Hrsg). EAES Guidelines for Endoscpic Surgery. Twelve Years Evidenced-Based Surgery in Europe. Berlin, Heidelberg: Springer, 2006: 291-295

206 Misra S, Chaturvedi A, Misra NC et al. Carcinoma of the gallbladder. Lancet Oncol 2003; 4: 167-176

207 Wiedmann M, Schoppmeyer K, Witzigmann H et al. Aktuelle Diagnostik und Therapie von Gallengangs- und Gallenblasenkarzinomen. Z Gastroenterol 2005; 43: 305-315

208 Kim EK, Lee SK, Kim WW. Does laparoscopic surgery have a role in the treatment of gallbladder cancer? J Hepatobiliary Pancreat Surg 2002; 9: 559-563

209 Shimizu T, Arima Y, Yokomuro S et al. Incidental gallbladder cancer diagnosed during and after laparoscopic cholecystectomy. J Nippon Med Sch 2006; 73: 136-140

210 Wakai T, Shirai Y, Yokoyama N et al. Early gallbladder carcinoma does not warrant radical resection. Br J Surg 2001; 88: 675-678

211 Ouchi K, Mikuni J, Kakugawa Y. Laparoscopic cholecystectomy for gallbladder carcinoma: results of a Japanese survey of 498 patients. J Hepatobiliary Pancreat Surg 2002; 9: 256-260

212 Yeh CN, Jan YY, Chen MF. Management of unsuspected gallbladder carcinoma discovered during or following laparoscopic cholecystectomy. Am Surg 2004; 70: 256-258

213 Sun $C D$, Zhang BY, Wu LQ et al. Laparoscopic cholecystectomy for treatment of unexpected early-stage gallbladder cancer. J Surg Oncol 2005; 91: 253-257

214 Kraas E, Frauenschuh D, Farke S. Intraoperative suspicion of gallbladder carcinoma in laparoscopic surgery: what to do? Dig Surg 2002; 19: 489-493

215 Wakai T, Shirai Y, Hatakeyama K. Radical second resection provides survival benefit for patients with T 2 gallbladder carcinoma first discovered after laparoscopic cholecystectomy. World J Surg 2002; 26: 867-871

216 Shirai Y, Yoshida K, Tsukada Ket al. Inapparent carcinoma of the gallbladder. An appraisal of a radical second operation after simple cholecystectomy. Ann Surg 1992; 215: 326-331

217 Yamaguchi K, Chijiiwa K, Saiki S et al. Retrospective analysis of 70 operations for gallbladder carcinoma. Br J Surg 1997; 84: 200-204

218 Suzuki S, Yokoi Y, Kurachi K et al. Appraisal of surgical treatment for pT2 gallbladder carcinomas. World J Surg 2004; 28: 160-165

219 Kondo S, Nimura Y, Hayakawa $N$ et al. Regional and para-aortic lymphadenectomy in radical surgery for advanced gallbladder carcinoma. Br J Surg 2000; 87: 418-422

220 Lundberg 0 , Kristoffersson A. Port site metastases from gallbladder cancer after laparoscopic cholecystectomy. Results of a Swedish survey and review of published reports. Eur J Surg 1999; 165: 215-222

221 Paolucci V, Schaeff B, Schneider M et al. Tumor seeding following laparoscopy: international survey. World J Surg 1999; 23: 989-995

222 Sicklick JK, Choti MA. Controversies in the surgical management of cholangiocarcinoma and gallbladder cancer. Semin Oncol 2005; 32: S112-S117

223 Takada T, Amano H, Yasuda $\mathrm{H}$ et al. Is postoperative adjuvant chemotherapy useful for gallbladder carcinoma? A phase III multicenter prospective randomized controlled trial in patients with resected pancreaticobiliary carcinoma. Cancer 2002; 95: 1685-1695

224 Kubicka S. Cholangiozelluläres Karzinom und Gallenblasenkarzinom. Z Gastroenterol 2004; 42: 397-402

225 Ko CW, Beresford SA, Schulte SJ et al. Incidence, natural history, and risk factors for biliary sludge and stones during pregnancy. Hepatology 2005; 41: 359-365

226 Ko CW. Risk factors for gallstone-related hospitalization during pregnancy and the postpartum. Am J Gastroenterol 2006; 101: 2263-2268

227 Hiatt JR, Hiatt JC, Williams RA et al. Biliary disease in pregnancy: strategy for surgical management. Am J Surg 1986; 151: 263-265

228 McKellar DP, Anderson CT, Boynton CJ et al. Cholecystectomy during pregnancy without fetal loss. Surg Gynecol Obstet 1992; 174 465-468 
229 Ghumman E, Barry M, Grace PA. Management of gallstones in pregnancy. Br J Surg 1997; 84: 1646-1650

$230 \mathrm{Lu}$ EJ, Curet MJ, El-Sayed YY et al. Medical versus surgical management of biliary tract disease in pregnancy. Am J Surg 2004; 188 : 755-759

231 Steinbrook RA, Brooks DC, Datta S. Laparoscopic cholecystectomy during pregnancy. Review of anesthetic management, surgical considerations. Surg Endosc 1996; 10: 511-515

232 Glasgow RE, Visser BC, Harris HW et al. Changing management of gallstone disease during pregnancy. Surg Endosc 1998; 12: 241-246

233 Cosenza CA, Saffari B, Jabbour $N$ et al. Surgical management of biliary gallstone disease during pregnancy. Am J Surg 1999; 178: 545-548

234 Neudecker J, Sauerland S, Neugebauer EA et al. The EAES clinical practice guidelines on the pneumoperitoenum for laparoscopic surgery (2002). In: Neugebauer EA, Sauerland S, Fingerhut A, Millat B, Buess G (Hrsg). EAES Guidelines for Endoscopic Surgery. Twelve Years Evidence-Based Surgery in Europe. Berlin, Heidelberg: Springer, 2006: 39-85

235 Curet MJ. Laparoscopy during pregnancy. In: Scott-Conner CE (Hrsg). The SAGES Manual. Fundamentals of Laparoscopy, Thoracoscopy, and GI Endoscopy. New York: Springer, 2006: 84-89

236 May GR, Sutherland LR, Shaffer EA. Efficacy of bile acid therapy for gallstone dissolution: a meta-analysis of randomized trials. Aliment Pharmacol Ther 1993; 7: 139-148

237 Petroni ML, Jazrawi RP, Pazzi P et al. Ursodeoxycholic acid alone or with chenodeoxycholic acid for dissolution of cholesterol gallstones: a randomized multicentre trial. The British-Italian Gallstone Study group. Aliment Pharmacol Ther 2001; 15: 123-128

238 Frigerio G. Ursodeoxycholic acid (UDCA) in the treatment of dyspepsia: report of a multicenter controlled trial. Current Therapeutic Research 1979; 26: 214-223

239 Inter-Hospital Clinical Research Group (G.I.R.C.). Effect of ursodeoxycholic acid (UDCA) on dyspesia in patients with gallstones or other biliary tract anomalies. Current Therapeutic Research 1979; 26: 230-233

240 Tomida S, Abei M, Yamaguchi Tet al. Long-term ursodeoxycholic acid therapy is associated with reduced risk of biliary pain and acute cholecystitis in patients with gallbladder stones: a cohort analysis. Hepatology 1999; 30: 6-13

241 Paumgartner G. Strategies in the treatment of gallstone disease. Working team report. Gastroenterology International 1993; 6: 65-75

242 Pauletzki J, Sailer C, Klüppelberg U et al. Gallbladder emptying determines early gallstone clearance after shock-wave lithotripsy. Gastroenterology 1994; 107: 1496-1502

243 Wermke W. Ultrasonic diagnosis of gallstone disease. Z Gesamte Inn Med 1989; 44: 377-382

244 Walters JR, Hood KA, Gleeson D et al. Combination therapy with oral ursodeoxycholic and chenodeoxycholic acids: pretreatment computed tomography of the gall bladder improves gall stone dissolution efficacy. Gut 1992; 33: 375-380

245 Jazrawi RP, Pigozzi MG, Galatola G et al. Optimum bile acid treatment for rapid gall stone dissolution. Gut 1992; 33: 381-386

246 Podda M, Zuin M, Battezzati PM et al. Efficacy and safety of a combination of chenodeoxycholic acid and ursodeoxycholic acid for gallstone dissolution: a comparison with ursodeoxycholic acid alone. Gastroenterology 1989; 96: 222-229

247 Janssen J, Johanns $W$, Weickert $U$ et al. Long-term results after successful extracorporeal gallstone lithotripsy: outcome of the first 120 stone-free patients. Scand J Gastroenterol 2001; 36: 314-317

248 Lanzini A, Jazrawi RP, Kupfer RM et al. Gallstone recurrence after medical dissolution. An overestimated threat? J Hepatol 1986; 3: 241-246

249 Petroni ML, Jazrawi RP, Goggin PM et al. Characteristics of recurrent gallstones following non-surgical treatment: implications for retreatment. Eur J Gastroenterol Hepatol 1991; 3: 473-478

250 Sackmann M, Pauletzki J, Aydemir U et al. Efficacy and safety of ursodeoxycholic acid for dissolution of gallstone fragments: comparison with the combination of ursodeoxycholic acid and chenodeoxycholic acid. Hepatology 1991; 14: 1136-1141

251 Sackmann M, Niller H, Klüppelberg $U$ et al. Gallstone recurrence after shock-wave therapy. Gastroenterology 1994; 106: 225-230

252 Pelletier G, Raymond JM, Capdeville R et al. Gallstone recurrence after successful lithotripsy. J Hepatol 1995; 23: 420-423
253 Cesmeli E, Elewaut AE, Kerre T et al. Gallstone recurrence after successful shock wave therapy: the magnitude of the problem and the predictive factors. Am J Gastroenterol 1999; 94: 474-479

254 Tsumita R, Sugiura N, Abe A et al. Long-term evaluation of extracorporeal shock-wave lithotripsy for cholesterol gallstones. J Gastroenterol Hepatol 2001; 16: 93-99

255 Rabenstein T, Radespiel-Tröger M, Höpfner $L$ et al. Ten years experience with piezoelectric extracorporeal shockwave lithotripsy of gallbladder stones. Eur J Gastroenterol Hepatol 2005; 17: 629-639

256 Venneman NG, van Berge-Henegouwen GP, Portincasa P et al. Absence of apolipoprotein E4 genotype, good gallbladder motility and presence of solitary stones delay rather than prevent gallstone recurrence after extracorporeal shock wave lithotripsy. J Hepatol 2001; 35: $10-16$

257 Carrilho-Ribeiro L, Pinto-Correia A, Velosa J et al. A ten-year prospective study on gallbladder stone recurrence after successful extracorporeal shock-wave lithotripsy. Scand J Gastroenterol 2006; 41: 338-342

258 Pauletzki J, Althaus R, Holl J et al. Gallbladder emptying and gallstone formation: a prospective study on gallstone recurrence. Gastroenterology 1996; 111: 765-771

259 Ochi H, Tazuma S, Kajihara T et al. Factors affecting gallstone recurrence after successful extracorporeal shock wave lithotripsy. J Clin Gastroenterol 2000; 31: 230-232

260 Petroni ML, Jazrawi RP, Pazzi P et al. Risk factors for the development of gallstone recurrence following medical dissolution. The BritishItalian Gallstone Study Group. Eur J Gastroenterol Hepatol 2000; 12: $695-700$

261 Tazuma S, Nishioka T, Ochi $\mathrm{H}$ et al. Impaired gallbladder mucosal function in aged gallstone patients suppresses gallstone recurrence after successful extracorporeal shockwave lithotripsy. J Gastroenterol Hepatol 2003; 18: 157-161

262 Adamek HE, Rochlitz C, von Bubnoff AC et al. Predictions and associations of cholecystectomy in patients with cholecystolithiasis treated with extracorporeal shock wave lithotripsy. Dig Dis Sci 2004; 49: 1938-1942

263 Sonnenberg A, Benninger J, Ell C. Kostenvergleich zwischen der laparoskopischen Cholezystektomie und der extrakorporalen Stoßwellenlithotripsie in der Behandlung von Gallenblasensteinen. Dtsch Med Wochenschr 1994; 119: 1532-1537

264 Go PM, Stolk MF, Obertop H et al. Symptomatic gallbladder stones. Cost-effectiveness of treatment with extracorporeal shock-wave lithotripsy, conventional and laparoscopic cholecystectomy. Surg Endosc 1995; 9: 37-41

265 Neubrand M, Holl J, Sackmann M et al. Combination of extracorporeal shock-wave lithotripsy and dissolution of gallbladder stones with methyl tert-butyl ether: a randomized study. Hepatology 1994; 19: 133-137

266 Pauletzki J, Holl J, Sackmann M et al. Gallstone recurrence after direct contact dissolution with methyl tert-butyl ether. Dig Dis Sci 1995; 40: 1775-1781

267 Hellstern A, Leuschner U, Benjaminov A et al. Dissolution of gallbladder stones with methyl-tert-butyl ether and stone recurrence: a European survey. Dig Dis Sci 1998; 43: 911-920

268 Maclure KM, Hayes KC, Colditz GA et al. Weight, diet, and the risk of symptomatic gallstones in middle-aged women. N Engl J Med 1989; 321: 563-569

269 Tsai CJ, Leitzmann MF, Willett WC et al. Central adiposity, regional fat distribution, and the risk of cholecystectomy in women. Gut 2006; 55: 708-714

270 Ortega RM, Fernandez-Azuela M, Encinas-Sotillos A et al. Differences in diet and food habits between patients with gallstones and controls. J Am Coll Nutr 1997; 16: 88-95

271 Leitzmann MF, Giovannucci EL, Rimm EB et al. The relation of physical activity to risk for symptomatic gallstone disease in men. Ann Intern Med 1998; 128: 417-425

272 Leitzmann MF, Rimm EB, Willett WC et al. Recreational physical activity and the risk of cholecystectomy in women. N Engl J Med 1999; 341: 777-784

273 Misciagna G, Centonze S, Leoci C et al. Diet, physical activity, and gallstones - a population-based, case-control study in southern Italy. Am J Clin Nutr 1999; 69: 120-126

274 Broomfield PH, Chopra R, Sheinbaum RC et al. Effects of ursodeoxycholic acid and aspirin on the formation of lithogenic bile and gallstones during loss of weight. N Engl J Med 1988; 319: 1567-1572 
275 Liddle RA, Goldstein RB, Saxton J. Gallstone formation during weightreduction dieting. Arch Intern Med 1989; 149: 1750-1753

276 Yang H, Petersen GM, Roth MP et al. Risk factors for gallstone formation during rapid loss of weight. Dig Dis Sci 1992; 37: 912-918

277 Weinsier RL, Wilson LJ, Lee J. Medically safe rate of weight loss for the treatment of obesity: a guideline based on risk of gallstone formation. Am J Med 1995; 98: 115-117

278 Syngal S, Coakley EH, Willett WC et al. Long-term weight patterns and risk for cholecystectomy in women. Ann Intern Med 1999; 130: 471-477

279 Tsai CJ, Leitzmann MF, Willett WC et al. Weight cycling and risk of gallstone disease in men. Arch Intern Med 2006; 166: 2369-2374

280 Williams C, Gowan R, Perey BJ. A double-blind placebo-controlled trial of ursodeoxycholic acid in the prevention of gallstones during weight loss after vertical banded gastroplasty. Obes Surg 1993; 3: 257-259

281 Worobetz LJ, Inglis FG, Shaffer EA. The effect of ursodeoxycholic acid therapy on gallstone formation in the morbidly obese during rapid weight loss. Am J Gastroenterol 1993; 88: 1705-1710

282 Shiffman ML, Kaplan GD, Brinkman-Kaplan $V$ et al. Prophylaxis against gallstone formation with ursodeoxycholic acid in patients participating in a very-low-calorie diet program. Ann Intern Med 1995; 122: 899-905

283 Sugerman HJ, Brewer WH, Shiffman ML et al. A multicenter, placebocontrolled, randomized, double-blind, prospective trial of prophylactic ursodiol for the prevention of gallstone formation following gastric-bypass-induced rapid weight loss. Am J Surg 1995; 169: 91-96

284 Wudel LJ, Wright JK, Debelak JP et al. Prevention of gallstone formation in morbidly obese patients undergoing rapid weight loss: results of a randomized controlled pilot study. J Surg Res 2002; 102: 50-56

285 Miller K, Hell E, Lang B et al. Gallstone formation prophylaxis after gastric restrictive procedures for weight loss: a randomized doubleblind placebo-controlled trial. Ann Surg 2003; 238: 697-702

286 Shoheiber O, Biskupiak JE, Nash DB. Estimation of the cost savings resulting from the use of ursodiol for the prevention of gallstones in obese patients undergoing rapid weight reduction. Int J Obes Relat Metab Disord 1997; 21: 1038-1045

287 Schmieder G, Stankov G, Zerle G et al. Observer-blind study with metamizole versus tramadol and butylscopolamine in acute biliary colic pain. Arzneimittelforschung 1993; 43: 1216-1221

288 Staritz M, Poralla T, Manns $M$ et al. Effect of modern analgesic drugs (tramadol, pentazocine, and buprenorphine) on the bile duct sphincter in man. Gut 1986; 27: 567-569

289 Cuer JC, Dapoigny M, Ajmi S et al. Effects of buprenorphine on motor activity of the sphincter of Oddi in man. Eur J Clin Pharmacol 1989; 36: 203-204

$290 \mathrm{Wu}$ SD, Zhang ZH, Jin JZ et al. Effects of narcotic analgesic drugs on human Oddi's sphincter motility. World J Gastroenterol 2004; 10 : 2901-2904

291 Hassel B. Treatment of biliary colic with nitroglycerin. Lancet 1993; 342: 1305

292 Goldman G, Kahn PJ, Alon R et al. Biliary colic treatment and acute cholecystitis prevention by prostaglandin inhibitor. Dig Dis Sci 1989; 34: 809-811

293 Akriviadis EA, Hatzigavriel M, Kapnias D et al. Treatment of biliary colic with diclofenac: a randomized, double-blind, placebo-controlled study. Gastroenterology 1997; 113: 225-231

294 Thornell E, Jansson R, Svanvik J. Indomethacin intravenously - a new way for effective relief of biliary pain: a double-blind study in man. Surgery 1981; 90: 468-472

295 Thornell E, Nilsson B, Jansson R et al. Effect of short-term indomethacin treatment on the clinical course of acute obstructive cholecystitis. Eur J Surg 1991; 157: 127-130

296 Kumar A, Deed JS, Bhasin B et al. Comparison of the effect of diclofenac with hyoscine-N-butylbromide in the symptomatic treatment of acute biliary colic. ANZ J Surg 2004; 74: 573-576

297 Shim CS, Joo JH, Park CW et al. Effectiveness of endoscopic ultrasonography in the diagnosis of choledocholithiasis prior to laparoscopic cholecystectomy. Endoscopy 1995; 27: 428-432

298 Sugiyama M, Atomi Y. Endoscopic ultrasonography for diagnosing choledocholithiasis: a prospective comparative study with ultrasonography and computed tomography. Gastrointest Endosc 1997; 45: $143-146$
299 Sugiyama M, Atomi Y. Acute biliary pancreatitis: the roles of endoscopic ultrasonography and endoscopic retrograde cholangiopancreatography. Surgery $1998 ; 124: 14-21$

300 Chak A, Hawes RH, Cooper GS et al. Prospective assessment of the utility of EUS in the evaluation of gallstone pancreatitis. Gastrointest Endosc 1999; 49: 599-604

301 Varghese JC, Liddell RP, Farrell MA et al. The diagnostic accuracy of magnetic resonance cholangiopancreatography and ultrasound compared with direct cholangiography in the detection of choledocholithiasis. Clin Radiol 1999; 54: 604-614

302 Cwik G, Wallner G, Ciechanski A et al. Endoscopic sphincterotomy in 100 patients scheduled for laparoscopic cholecystectomy: ultrasound evaluation. Hepatogastroenterology 2003; 50: 1225-1228

303 Rickes S, Treiber G, Mönkemüller K et al. Impact of the operator's experience on value of high-resolution transabdominal ultrasound in the diagnosis of choledocholithiasis: a prospective comparison using endoscopic retrograde cholangiography as the gold standard. Scand J Gastroenterol 2006; 41: 838-843

304 Thornton JR, Lobo AJ, Lintott DJ et al. Value of ultrasound and liver function tests in determining the need for endoscopic retrograde cholangiopancreatography in unexplained abdominal pain. Gut 1992; 33: 1559-1561

305 Majeed AW, Ross B, Johnson AG et al. Common duct diameter as an independent predictor of choledocholithiasis: is it useful? Clin Radiol 1999; 54: 170-172

306 Aronson N, Flamm CR, Mark D et al. Endoscopic retrograde cholangiopancreatography. Evid Rep Technol Assess 2002; 50: 1-369

307 Barkun AN, Barkun JS, Fried GM et al. Useful predictors of bile duct stones in patients undergoing laparoscopic cholecystectomy. McGill Gallstone Treatment Group. Ann Surg 1994; 220: 32-39

308 Koo KP, Traverso LW. Do preoperative indicators predict the presence of common bile duct stones during laparoscopic cholecystectomy? Am J Surg 1996; 171: 495-499

309 Onken JE, Brazer SR, Eisen GM et al. Predicting the presence of choledocholithiasis in patients with symptomatic cholelithiasis. Am J Gastroenterol 1996; 91: 762-767

310 Santucci L, Natalini G, Sarpi L et al. Selective endoscopic retrograde cholangiography and preoperative bile duct stone removal in patients scheduled for laparoscopic cholecystectomy: a prospective study. Am J Gastroenterol 1996; 91: 1326-1330

311 Alponat A, Kum CK, Rajnakova A et al. Predictive factors for synchronous common bile duct stones in patients with cholelithiasis. Surg Endosc 1997; 11: 928-932

$312 \mathrm{Kim} \mathrm{KH,} \mathrm{Kim} \mathrm{W,} \mathrm{Lee} \mathrm{HI} \mathrm{et} \mathrm{al.} \mathrm{Prediction} \mathrm{of} \mathrm{common} \mathrm{bile} \mathrm{duct} \mathrm{stones:}$ its validation in laparoscopic cholecystectomy. Hepatogastroenterology 1997; 44: 1574-1579

313 Bergamaschi R, Tuech JJ, Braconier L et al. Selective endoscopic retrograde cholangiography prior to laparoscopic cholecystectomy for gallstones. Am J Surg 1999; 178: 46-49

314 Masci E, Fanti L, Mariani A et al. Selection criteria for pre-operative endoscopic retrograde cholangiography and endoscopic-laparoscopic treatment of biliary stones. Eur J Gastroenterol Hepatol 1999; 11: 781-784

315 Prat F, Meduri B, Ducot B et al. Prediction of common bile duct stones by noninvasive tests. Ann Surg 1999; 229: 362-368

316 Sahai AV, Mauldin PD, Marsi V et al. Bile duct stones and laparoscopic cholecystectomy: a decision analysis to assess the roles of intraoperative cholangiography, EUS, and ERCP. Gastrointest Endosc 1999; 49: 334-343

317 Kama NA, Atli M, Doganay $M$ et al. Practical recommendations for the prediction and management of common bile duct stones in patients with gallstones. Surg Endosc 2001; 15: 942-945

318 Liu TH, Consorti ET, Kawashima A et al. Patient evaluation and management with selective use of magnetic resonance cholangiography and endoscopic retrograde cholangiopancreatography before laparoscopic cholecystectomy. Ann Surg 2001; 234: 33-40

319 Luman W, Chan ES. Mathematical model for predicting biliary therapeutic endoscopic retrograde pancreatography (ERCP). Dig Liver Dis 2003; 35: 486-492

320 Sarli L, Costi R, Gobbi S et al. Scoring system to predict asymptomatic choledocholithiasis before laparoscopic cholecystectomy. A matched case-control study. Surg Endosc 2003; 17: 1396-1403

321 Cohen S, Bacon BR, Berlin JA et al. NIH state-of-the-science statement on endoscopic retrograde cholangiopancreatography (ERCP) for di- 
agnosis and therapy. NIH Consensus State Sci Statements 2002; 19: $1-26$

322 Palazzo L, Girollet PP, Salmeron M et al. Value of endoscopic ultrasonography in the diagnosis of common bile duct stones: comparison with surgical exploration and ERCP. Gastrointest Endosc 1995; 42: 225-231

323 Liu CL, Lo CM, Chan JK et al. Detection of choledocholithiasis by EUS in acute pancreatitis: a prospective evaluation in 100 consecutive patients. Gastrointest Endosc 2001; 54: 325-330

324 Tseng LJ, Jao YT, Mo LR et al. Over-the-wire US catheter probe as an adjunct to ERCP in the detection of choledocholithiasis. Gastrointest Endosc 2001; 54: 720-723

325 Moon JH, Cho YD, Cha SW et al. The detection of bile duct stones in suspected biliary pancreatitis: comparison of MRCP, ERCP, and intraductal US. Am J Gastroenterol 2005; 100: 1051-1057

326 Johnson AG, Hosking SW. Appraisal of the management of bile duct stones. Br J Surg 1987; 74: 555-560

327 Murison MS, Gartell PC, McGinn FP. Does selective peroperative cholangiography result in missed common bile duct stones? J R Coll Surg Edinb 1993; 38: 220-224

328 Dancygier $H$, Nattermann $C$. The role of endoscopic ultrasonography in biliary tract disease: obstructive jaundice. Endoscopy 1994; 26 : 800-802

329 Prat F, Amouyal G, Amouyal P et al. Prospective controlled study of endoscopic ultrasonography and endoscopic retrograde cholangiography in patients with suspected common-bileduct lithiasis. Lancet 1996; 347: 75-79

330 Norton SA, Alderson D. Prospective comparison of endoscopic ultrasonography and endoscopic retrograde cholangiopancreatography in the detection of bile duct stones. Br J Surg 1997; 84: 1366-1369

331 Canto MI, Chak A, Stellato T et al. Endoscopic ultrasonography versus cholangiography for the diagnosis of choledocholithiasis. Gastrointest Endosc 1998; 47: 439-448

332 De Ledinghen $V$, Lecesne R, Raymond JM et al. Diagnosis of choledocholithiasis: EUS or magnetic resonance cholangiography? A prospective controlled study. Gastrointest Endosc 1999; 49: 26-31

333 Polkowski M, Palucki J, Regula J et al. Helical computed tomographic cholangiography versus endosonography for suspected bile duct stones: a prospective blinded study in non-jaundiced patients. Gut 1999; 45: 744-749

334 Materne R, van Beers BE, Gigot JF et al. Extrahepatic biliary obstruction: magnetic resonance imaging compared with endoscopic ultrasonography. Endoscopy 2000; 32: 3-9

335 Scheiman JM, Carlos RC, Barnett JL et al. Can endoscopic ultrasound or magnetic resonance cholangiopancreatography replace ERCP in patients with suspected biliary disease? A prospective trial and cost analysis. Am J Gastroenterol 2001; 96: 2900-2904

336 Ainsworth AP, Rafaelsen SR, Wamberg PA et al. Is there a difference in diagnostic accuracy and clinical impact between endoscopic ultrasonography and magnetic resonance cholangiopancreatography? Endoscopy 2003; 35: 1029-1032

337 Buscarini E, Tansini P, Vallisa D et al. EUS for suspected choledocholithiasis: do benefits outweigh costs? A prospective, controlled study. Gastrointest Endosc 2003; 57: 510-518

338 Napoleon B, Dumortier J, Keriven-Souquet 0 et al. Do normal findings at biliary endoscopic ultrasonography obviate the need for endoscopic retrograde cholangiography in patients with suspicion of common bile duct stone? A prospective follow-up study of 238 patients. Endoscopy 2003; 35: 411-415

339 Thorboll J, Vilmann P, Jacobsen B et al. Endoscopic ultrasonography in detection of cholelithiasis in patients with biliary pain and negative transabdominal ultrasonography. Scand J Gastroenterol 2004; 39 : 267-269

340 Tse F, Barkun JS, Barkun AN. The elective evaluation of patients with suspected choledocholithiasis undergoing laparoscopic cholecystectomy. Gastrointest Endosc 2004; 60: 437-448

341 Aubé C, Delorme B, Yzet T et al. MR cholangiopancreatography versus endoscopic sonography in suspected common bile duct lithiasis: a prospective, comparative study. Am J Roentgenol 2005; 184: 55-62

342 Kondo $S$, Isayama $H$, Akahane $M$ et al. Detection of common bile duct stones: comparison between endoscopic ultrasonography, magnetic resonance cholangiography, and helical-computed-tomographic cholangiography. Eur J Radiol 2005; 54: 271-275

343 Mirbagheri SA, Mohamadnejad M, Nasiri J et al. Prospective evaluation of endoscopic ultrasonography in the diagnosis of biliary micro- lithiasis in patients with normal transabdominal ultrasonography. J Gastrointest Surg 2005; 9: 961-964

344 Ney MV, Maluf-Filho F, Sakai P et al. Echo-endoscopy versus endoscopic retrograde cholangiography for the diagnosis of choledocholithiasis: the influence of the size of the stone and diameter of the common bile duct. Arq Gastroenterol 2005; 42: 239-243

345 Guibaud L, Bret PM, Reinhold C et al. Bile duct obstruction and choledocholithiasis: diagnosis with MR cholangiography. Radiology 1995; 197: 109-115

346 Chan YL, Chan AC, Lam WW et al. Choledocholithiasis: comparison of MR cholangiography and endoscopic retrograde cholangiography. Radiology 1996; 200: 85-89

347 Reuther G, Kiefer B, Tuchmann A et al. MR-Cholangiopankreatikografie als Einzelschussprojektion: Erfahrungen und Ergebnisse bei 200 Untersuchungen. Fortschr Röntgenstr 1996; 165: 535-543

348 Pavone P, Laghi A, Lomanto D et al. MR cholangiography (MRC) in the evaluation of CBD stones before laparoscopic cholecystectomy. Surg Endosc 1997; 11: 982-985

349 Adamek HE, Albert J, Weitz $M$ et al. A prospective evaluation of magnetic resonance cholangiopancreatography in patients with suspected bile duct obstruction. Gut 1998; 43: 680-683

350 Holzknecht N, Gauger J, Sackmann M et al. Breath-hold MR cholangiography with snapshot techniques: prospective comparison with endoscopic retrograde cholangiography. Radiology 1998; 206: 657-664

351 Lomas DJ, Bearcroft PW, Gimson AE. MR cholangiopancreatography: prospective comparison of a breath-hold 2D projection technique with diagnostic ERCP. Eur Radiol 1999; 9: 1411-1417

352 Zidi SH, Prat F, Le Guen O et al. Use of magnetic resonance cholangiography in the diagnosis of choledocholithiasis: prospective comparison with a reference imaging method. Gut 1999; 44: 118-122

353 Angulo P, Pearce DH, Johnson CD et al. Magnetic resonance cholangiography in patients with biliary disease: its role in primary sclerosing cholangitis. J Hepatol 2000; 33: 520-527

354 Demartines N, Eisner L, Schnabel Ket al. Evaluation of magnetic resonance cholangiography in the management of bile duct stones. Arch Surg 2000; 135: 148-152

355 Soto JA, Alvarez O, Munera F et al. Diagnosing bile duct stones: comparison of unenhanced helical CT, oral contrast-enhanced CT cholangiography, and MR cholangiography. Am J Roentgenol 2000; 175: 1127-1134

356 Soto JA, Barish MA, Alvarez 0 et al. Detection of choledocholithiasis with MR cholangiography: comparison of three-dimensional fast spin-echo and single- and multisection half-Fourier rapid acquisition with relaxation enhancement sequences. Radiology 2000; 215 : 737-745

357 Stiris MG, Tennoe B, Aadland E et al. MR cholangiopancreaticography and endoscopic retrograde cholangiopancreaticography in patients with suspected common bile duct stones. Acta Radiol 2000; 41: 269-272

358 Laokpessi A, Bouillet P, Sautereau D et al. Value of magnetic resonance cholangiography in the preoperative diagnosis of common bile duct stones. Am J Gastroenterol 2001; 96: 2354-2359

359 Boraschi P, Gigoni R, Braccini $G$ et al. Detection of common bile duct stones before laparoscopic cholecystectomy. Evaluation with MR cholangiography. Acta Radiol 2002; 43: 593-598

360 Calvo MM, Bujanda L, Calderon A et al. Role of magnetic resonance cholangiopancreatography in patients with suspected choledocholithiasis. Mayo Clin Proc 2002; 77: 422-428

361 Hakansson K, Ekberg O, Hakansson $\mathrm{HO}$ et al. MR and ultrasound in screening of patients with suspected biliary tract disease. Acta Radiol 2002; 43: 80-86

362 Hussein FM, Alsumait B, Aman S et al. Diagnosis of choledocholithiasis and bile duct stenosis by magnetic resonance cholangiogram. Australas Radiol 2002; 46: 41-46

363 Jendresen MB, Thorboll JE, Adamsen S et al. Preoperative routine magnetic resonance cholangiopancreatography before laparoscopic cholecystectomy: a prospective study. Eur J Surg 2002; 168: 690-694

$364 \mathrm{Kim}$ TK, Kim BS, Kim JH et al. Diagnosis of intrahepatic stones: superiority of MR cholangiopancreatography over endoscopic retrograde cholangiopancreatography. Am J Roentgenol 2002; 179: 429-434

$365 \mathrm{Kim}$ JH, Kim MJ, Park SI et al. MR cholangiography in symptomatic gallstones: diagnostic accuracy according to clinical risk group. Radiology 2002; 224: 410-416 
366 Taylor AC, Little AF, Hennessy OF et al. Prospective assessment of magnetic resonance cholangiopancreatography for noninvasive imaging of the biliary tree. Gastrointest Endosc 2002; 55: 17-22

367 Urban M, Holzer B, Sebesta C et al. Efficacy of diagnosis of mechanical cholestasis by magnetic resonance cholangiography. World J Surg 2002; 26: 353-358

368 Griffin N, Wastle ML, Dunn WK et al. Magnetic resonance cholangiopancreatography versus endoscopic retrograde cholangiopancreatography in the diagnosis of choledocholithiasis. Eur J Gastroenterol Hepatol 2003; 15: 809-813

369 Kats J, Kraai M, Dijkstra AJ et al. Magnetic resonance cholangiopancreaticography as a diagnostic tool for common bile duct stones: a comparison with ERCP and clinical follow-up. Dig Surg 2003; 20: $32-37$

370 Romagnuolo J, Bardou M, Rahme E et al. Magnetic resonance cholangiopancreatography: a meta-analysis of test performance in suspected biliary disease. Ann Intern Med 2003; 139: 547-557

371 Kaltenthaler E, Vergel YB, Chilcott J et al. A systematic review and economic evaluation of magnetic resonance cholangiopancreatography compared with diagnostic endoscopic retrograde cholangiopancreatography. Health Technol Assess 2004; 8: 1-89

372 Verma D, Kapedia A, Eisen GM et al. EUS vs MRCP for detection of choledocholithiasis. Gastrointest Endosc 2006; 64: 248-254

373 Varadarajulu S, Eloubeidi MA, Wilcox CM et al. Do all patients with abnormal intraoperative cholangiogram merit endoscopic retrograde cholangiopancreatography? Surg Endosc 2006; 20: 801-805

374 Ludwig K, Bernhardt J, Lorenz D. Value and consequences of routine intraoperative cholangiography during cholecystectomy. Surg Laparosc Endosc Percutan Tech 2002; 12: 154-159

375 Ludwig K, Wilhelm L, Prinz C et al. Die intraoperative laparoskopische Cholangiografie - Wann ist sie sinnvoll? Zentralbl Chir 2004; 129: 185-190

376 Sauerbruch T, Stellaard F, Soehendra $N$ et al. Cholesteringehalt von Gallengangsteinen. Dtsch Med Wochenschr 1983; 108: 1099-1102

377 Cahalane MJ, Neubrand MW, Carey MC. Physical-chemical pathogenesis of pigment gallstones. Semin Liver Dis 1988; 8: 317-328

378 Thistle JL. Pathophysiology of bile duct stones. World J Surg 1998; 22: 1114-1118

379 Neubrand M, Sauerbruch T. Pathogenese der Choledocholithiasis. Chir Gastroenterol 2001; 17: 119-122

380 Lacaine F, Corlette MB, Bismuth $H$. Preoperative evaluation of the risk of common bile duct stones. Arch Surg 1980; 115: 1114-1116

381 Saltzstein EC, Peacock JB, Thomas MD. Preoperative bilirubin, alkaline phosphatase and amylase levels as predictors of common duct stones. Surg Gynecol Obstet 1982; 154: 381-384

382 Neuhaus H, Feussner H, Ungeheuer A et al. Prospective evaluation of the use of endoscopic retrograde cholangiography prior to laparoscopic cholecystectomy. Endoscopy 1992; 24: 745-749

383 Houdart R, Perniceni T, Darne B et al. Predicting common bile duct lithiasis: determination and prospective validation of a model predicting low risk. Am J Surg 1995; 170: 38-43

384 Welbourn CR, Mehta D, Armstrong CP et al. Selective preoperative endoscopic retrograde cholangiography with sphincterotomy avoids bile duct exploration during laparoscopic cholecystectomy. Gut 1995; 37: 576-579

385 Collins C, Maguire D, Ireland A et al. A prospective study of common bile duct calculi in patients undergoing laparoscopic cholecystectomy: natural history of choledocholithiasis revisited. Ann Surg 2004; 239: 28-33

386 Millbourn E. Klinische Studien über die Choledocholithiasis unter besonderer Berücksichtigung des Ikterus, des Verhaltens der Diastasurie des spontanen Steinabgangs, sowie der Früh- und Spätergebnisse nach konservativer und operativer Therapie. Acta Chir Scand 1941; 86: 1-310

387 Frossard JL, Hadengue A, Amouyal G et al. Choledocholithiasis: a prospective study of spontaneous common bile duct stone migration. Gastrointest Endosc 2000; 51: 175-179

388 Caddy GR, Kirby J, Kirk SJ et al. Natural history of asymptomatic bile duct stones at time of cholecystectomy. Ulster Med J 2005; 74: $108-112$

389 Acosta MJ, Rossi R, Ledesma CL. The usefulness of stool screening for diagnosing cholelithiasis in acute pancreatitis. A description of the technique. Am J Dig Dis 1977; 22: 168-172
390 Greiner L, Münks C, Heil W et al. Gallbladder stone fragments in feces after biliary extracorporeal shock-wave lithotripsy. Gastroenterology 1990; 98: 1620-1624

391 Classen M, Demling L. Endoskopische Sphinkterotomie der Papilla Vateri und Steinextraktion aus dem Ductus choledochus. Dtsch Med Wochenschr 1974; 99: 469-467

392 Cotton PB. Endoscopic management of bile duct stones (apples and oranges). Gut 1984; 25: 587-597

393 Miller BM, Kozarek RA, Ryan JA et al. Surgical versus endoscopic management of common bile duct stones. Ann Surg 1988; 207: 135-141

394 Targarona EM, Ayuso RM, Bordas JM et al. Randomised trial of endoscopic sphincterotomy with gallbladder left in situ versus open surgery for common bileduct calculi in high-risk patients. Lancet 1996; 347: 926-929

395 Kautz G. Indikationen, Befunde und Komplikationen der ERCP in der Pädiatrie. Z Gastroenterol 1993; 31: 742-750

396 Deenitchin GP, Konomi H, Kimura H et al. Reappraisal of safety of endoscopic sphincterotomy for common bile duct stones in the elderly. Am J Surg 1995; 170: 51-54

397 Deans GT, Sedman P, Martin DF et al. Are complications of endoscopic sphincterotomy age related? Gut 1997; 41: 545-548

398 Tham TC, Carr-Locke DL, Collins JS. Endoscopic sphincterotomy in the young patient: is there cause for concern? Gut 1997; 40: 697-700

399 Shuchleib S, Chousleb A, Mondragon A et al. Laparoscopic common bile duct exploration. World J Surg 1999; 23: 698-701

400 Daradkeh S, Shennak M, Abu-Khalaf M. Selective use of perioperative ERCP in patients undergoing laparoscopic cholecystectomy. Hepatogastroenterology 2000; 47: 1213-1215

401 Biffl WL, Moore EE, Offner PJ et al. Routine intraoperative laparoscopic ultrasonography with selective cholangiography reduces bile duct complications during laparoscopic cholecystectomy. J Am Coll Surg 2001; 193: 272-280

402 Iodice G, Giardiello C, Francica $G$ et al. Single-step treatment of gallbladder and bile duct stones: a combined endoscopic-laparoscopic technique. Gastrointest Endosc 2001; 53: 336-338

403 Lauschke H, Kaminski M, Verfurth B et al. Die Choledochusrevision im Zeitalter der endoskopischen Papillotomie. Indikationen und Ergebnisse. Zentralbl Chir 2001; 126: 364-368

404 Leibl BJ, Ulrich M, Scheuritzel U et al. Selektive prä-, intra- und postoperative Gallengangdiagnostik - Ein effizientes und komplikationsarmes Regime im Rahmen der laparoskopischen Cholecystektomie. Früh- und Spätergebnisse einer prospektiven Studie. Chirurg 2001; 72: 812-817

405 Urbach DR, Khajanchee YS, Jobe BA et al. Cost-effective management of common bile duct stones: a decision analysis of the use of endoscopic retrograde cholangiopancreatography (ERCP), intraoperative cholangiography, and laparoscopic bile duct exploration. Surg Endosc 2001; 15: 4-13

406 Meyer C, Le JV, Rohr S et al. Management of common bile duct stones in a single operation combining laparoscopic cholecystectomy and perioperative endoscopic sphincterotomy. Surg Endosc 1999; 13 : 874-877

407 Thielemann H, Laun C, Naveke R et al. Das diagnostische und therapeutische Management bei symptomatischer Cholezystolithiasis und präoperativ bestehendem Verdacht auf eine Choledocholithiasis. Zentralbl Chir 2002; 127: 987-991

408 Williams GL, Vellacott KD. Selective operative cholangiography and perioperative endoscopic retrograde cholangiopancreatography (ERCP) during laparoscopic cholecystectomy: a viable option for choledocholithiasis. Surg Endosc 2002; 16: 465-467

409 Hamy A, Hennekinne S, Pessaux P et al. Endoscopic sphincterotomy prior to laparoscopic cholecystectomy for the treatment of cholelithiasis. Surg Endosc 2003; 17: 872-875

410 Enochsson L, Lindberg B, Swahn F et al. Intraoperative endoscopic retrograde cholangiopancreatography (ERCP) to remove common bile duct stones during routine laparoscopic cholecystectomy does not prolong hospitalization: a 2-year experience. Surg Endosc 2004; 18 : 367-371

411 Hüttl TP, Hrdina C, Geiger TK et al. Management der Choledocholithiasis. Umfrageergebnisse und Analyse von 8433 Gallengangsrevisionen. Zentralbl Chir 2002; 127: 282-288

412 Martin DJ, Vernon DR, Toouli J. Surgical versus endoscopic treatment of bile duct stones. Cochrane Database Syst Rev 2006; CD003327 
413 Clayton ES, Connor S, Alexakis N et al. Meta-analysis of endoscopy and surgery versus surgery alone for common bile duct stones with the gallbladder in situ. Br J Surg 2006; 93: 1185-1191

414 Neoptolemos JP, Carr-Locke DL, Fossard DP. Prospective randomised study of preoperative endoscopic sphincterotomy versus surgery alone for common bile duct stones. Br Med J 1987; 294: 470-474

415 Stain SC, Cohen H, Tsuishoysha M et al. Choledocholithiasis. Endoscopic sphincterotomy or common bile duct exploration. Ann Surg 1991; 213: 627-633

416 Stiegmann GV, GoffJS, Mansour A et al. Precholecystectomy endoscopic cholangiography and stone removal is not superior to cholecystectomy, cholangiography, and common duct exploration. Am J Surg 1992; 163: 227-230

417 Hammarström LE, Holmin T, Stridbeck H et al. Long-term follow-up of a prospective randomized study of endoscopic versus surgical treatment of bile duct calculi in patients with gallbladder in situ. Br J Surg 1995; 82: 1516-1521

418 Kapoor R, Kaushik SP, Saraswat VA et al. Prospective randomized trial comparing endoscopic sphincterotomy followed by surgery with surgery alone in good risk patients with choledocholithiasis. HPB Surg 1996; 9: 145-148

419 Suc B, Escat J, Cherqui D et al. Surgery vs endoscopy as primary treatment in symptomatic patients with suspected common bile duct stones: a multicenter randomized trial. Arch Surg 1998; 133: 702-708

420 Cuschieri A, Lezoche E, Morino M et al. E. A. E. S. multicenter prospective randomized trial comparing two-stage vs single-stage management of patients with gallstone disease and ductal calculi. Surg Endosc 1999; 13: 952-957

421 Sgourakis G, Karaliotas K. Laparoscopic common bile duct exploration and cholecystectomy versus endoscopic stone extraction and laparoscopic cholecystectomy for choledocholithiasis. A prospective randomized study. Minerva Chir 2002; 57: 467-474

422 Rhodes M, Sussman L, Cohen L et al. Randomised trial of laparoscopic exploration of common bile duct versus postoperative endoscopic retrograde cholangiography for common bile duct stones. Lancet 1998: 351: 159-161

423 Nathanson LK, O'Rourke NA, Martin IJ et al. Postoperative ERCP versus laparoscopic choledochotomy for clearance of selected bile duct calculi: a randomized trial. Ann Surg 2005; 242: 188-192

424 Poulose BK, Arbogast PG, Holzman MD. National analysis of in-hospital resource utilization in choledocholithiasis management using propensity scores. Surg Endosc 2006; 20: 186-190

425 Ammori BJ, Birbas K, Davides D et al. Routine vs „on demand“ postoperative ERCP for small bile duct calculi detected at intraoperative cholangiography. Clinical evaluation and cost analysis. Surg Endosc 2000; 14: 1123-1126

426 Basso N, Pizzuto G, Surgo D et al. Laparoscopic cholecystectomy and intraoperative endoscopic sphincterotomy in the treatment of cholecysto-choledocholithiasis. Gastrointest Endosc 1999; 50: 532-535

427 Meyer C, Le JV, Rohr S et al. Management of common bile duct stones in a single operation combining laparoscopic cholecystectomy and peroperative endoscopic sphincterotomy. J Hepatobiliary Pancreat Surg 2002; 9: 196-200

428 Wei Q Wang JG, Li LB et al. Management of choledocholithiasis: comparison between laparoscopic common bile duct exploration and intraoperative endoscopic sphincterotomy. World J Gastroenterol 2003; 9: 2856-2858

429 Lella F, Bagnolo F, Rebuffat C et al. Use of the laparoscopic-endoscopic approach, the so-called „rendezvous“ technique, in cholecystocholedocholithiasis: a valid method in cases with patient-related risk factors for post-ERCP pancreatitis. Surg Endosc 2006; 20: 419-423

430 Rabago LR, Vicente C, Soler F et al. Two-stage treatment with preoperative endoscopic retrograde cholangiopancreatography (ERCP) compared with single-stage treatment with intraoperative ERCP for patients with symptomatic cholelithiasis with possible choledocholithiasis. Endoscopy 2006; 38: 779-786

431 Metcalfe MS, Ong T, Bruening MH et al. Is laparoscopic intraoperative cholangiogram a matter of routine? Am J Surg 2004; 187: 475-481

432 Järhult J. Is preoperative evaluation of the biliary tree necessary in uncomplicated gallstone disease? Results of a randomized trial. Scand J Surg 2005; 94: 31-33

433 Hawasli A, Lloyd L, Pozios $V$ et al. The role of endoscopic retrograde cholangio-pancreaticrogram in laparoscopic cholecystectomy. Am Surg 1993; 59: 285-288
434 Trondsen E, Edwin B, Reiertsen $O$ et al. Selection criteria for endoscopic retrograde cholangiopancreaticography (ERCP) in patients with gallstone disease. World J Surg 1995; 19: 852-856

435 Menezes N, Marson LP, Debeaux AC et al. Prospective analysis of a scoring system to predict choledocholithiasis. Br J Surg 2000; 87: 1176-1181

436 Katz D, Nikfarjam M, Sfakiotaki A et al. Selective endoscopic cholangiography for the detection of common bile duct stones in patients with cholelithiasis. Endoscopy 2004; 36: 1045-1049

437 Bachar GN, Cohen M, Belenky A et al. Effect of aging on the adult extrahepatic bile duct: a sonographic study. J Ultrasound Med 2003; 22: 879-882

438 Cotton PB. Endoscopic retrograde cholangiopancreatography and laparoscopic cholecystectomy. Am J Surg 1993; 165: 474-478

439 Van der Velden JJ, Berger MY, Bonjer HJ et al. Percutaneous treatment of bile duct stones in patients treated unsuccessfully with endoscopic retrograde procedures. Gastrointest Endosc 2000; 51: 418-422

440 Garcia-Vila JH, Redondo-Ibanez M, Diaz-Ramon C. Balloon sphincteroplasty and transpapillary elimination of bile duct stones: 10 years' experience. Am J Roentgenol 2004; 182: 1451-1458

441 Chespak LW, Ring EJ, Shapiro HA et al. Multidisciplinary approach to complex endoscopic biliary intervention. Radiology 1989; 170: 995-997

442 Navarrete CG, Castillo CT, Castillo PY. Choledocholithiasis: percutaneous treatment. World J Surg 1998; 22: 1151-1154

443 Stage JG, Moesgaard F, Gronvall S et al. Percutaneous transhepatic cholelithotripsy for difficult common bile duct stones. Endoscopy 1998; 30: 289-292

444 Picus D, Weyman PJ, Marx MV. Role of percutaneous intracorporeal electrohydraulic lithotripsy in the treatment of biliary tract calculi. Work in progress. Radiology 1989; 170: 989-993

445 Bonnel DH, Liguory CE, Cornud FE et al. Common bile duct and intrahepatic stones: results of transhepatic electrohydraulic lithotripsy in 50 patients. Radiology 1991; 180: 345-348

446 Hayashi N, Sakai T, Yamamoto T et al. Percutaneous transhepatic lithotripsy using a choledochoscope: long-term follow-up in 14 patients. Am J Roentgenol 1998; 171: 1387-1389

447 Demling L, Ermert H, Riemann JF et al. Lithotripsy in the common bile duct using ultrasound. Preliminary in vitro experiments. Endoscopy 1984; 16: 226-228

448 Garg PK, Tandon RK, Ahuja V et al. Predictors of unsuccessful mechanical lithotripsy and endoscopic clearance of large bile duct stones. Gastrointest Endosc 2004; 59: 601-605

449 Chang WH, Chu CH, Wang TE et al. Outcome of simple use of mechanical lithotripsy of difficult common bile duct stones. World J Gastroenterol 2005; 11: 593-596

450 Jakobs R, Adamek HE, Maier M et al. Fluoroscopically guided laser lithotripsy versus extracorporeal shock wave lithotripsy for retained bile duct stones: a prospective randomised study. Gut 1997; 40: 678-682

451 Neuhaus H, Zillinger C, Born P et al. Randomized study of intracorporeal laser lithotripsy versus extracorporeal shock-wave lithotripsy for difficult bile duct stones. Gastrointest Endosc 1998; 47: 327-334

452 Sauerbruch T, Stern M. Fragmentation of bile duct stones by extracorporeal shock waves. A new approach to biliary calculi after failure of routine endoscopic measures. Gastroenterology 1989; 96: 146-152

453 Sauerbruch T, Holl J, Sackmann $M$ et al. Fragmentation of bile duct stones by extracorporeal shock-wave lithotripsy: a five-year experience. Hepatology 1992; 15: 208-214

454 Adamek HE, Buttmann A, Wessbecher R et al. Clinical comparison of extracorporeal piezoelectric lithotripsy (EPL) and intracorporeal electrohydraulic lithotripsy (EHL) in difficult bile duct stones. A prospective randomized trial. Dig Dis Sci 1995; 40: 1185-1192

455 Adamek HE, Maier M, Jakobs $R$ et al. Management of retained bile duct stones: a prospective open trial comparing extracorporeal and intracorporeal lithotripsy. Gastrointest Endosc 1996; 44: 40-47

456 Ellis RD, Jenkins AP, Thompson RP et al. Clearance of refractory bile duct stones with extracorporeal shockwave lithotripsy. Gut 2000; 47: 728-731

457 Sackmann M, Holl J, Sauter GH et al. Extracorporeal shock wave lithotripsy for clearance of bile duct stones resistant to endoscopic extraction. Gastrointest Endosc 2001; 53: 27-32

458 Adamek HE, Kudis V, Jakobs $R$ et al. Impact of gallbladder status on the outcome in patients with retained bile duct stones treated with 
extracorporeal shockwave lithotripsy. Endoscopy 2002; 34 : 624-627

459 Riemann JF, Lux G, Forster P et al. Long-term results after endoscopic papillotomy. Endoscopy 1983; 15 (Suppl 1): 165-168

460 Binmoeller KF, Brückner M, Thonke F et al. Treatment of difficult bile duct stones using mechanical, electrohydraulic and extracorporeal shock wave lithotripsy. Endoscopy 1993; 25: 201-206

461 Ell C, Lux G, Hochberger J et al. Laserlithotripsy of common bile duct stones. Gut 1988; 29: 746-751

462 Behjou B, Prat F, Fritsch J et al. Intra-corporeal shockwave lithotripsy in the treatment of complex lithiasis of the bile ducts. Comparison of endoscopic techniques and long-term results. Gastroenterol Clin Biol 1997; 21: 648-654

463 Hochberger J, Bayer J, May A et al. Laser lithotripsy of difficult bile duct stones: results in 60 patients using a rhodamine 6G dye laser with optical stone tissue detection system. Gut 1998; 43: 823-829

464 Palmer K, Hofmann A. Intraductal mono-octanoin for the direct dissolution of bile duct stones: experience in 343 patients. Gut 1986; 27: 196-202

465 Leuschner $U$. Topical treatment of calcified and pigment stones. Semin Liver Dis 1990; 10: 191-196

466 Minami A, Nakatsu T, Uchida N et al. Papillary dilation vs sphincterotomy in endoscopic removal of bile duct stones. A randomized trial with manometric function. Dig Dis Sci 1995; 40: 2550-2554

467 Bergman JJ, Rauws EA, Fockens P et al. Randomised trial of endoscopic balloon dilation versus endoscopic sphincterotomy for removal of bileduct stones. Lancet 1997; 349: 1124-1129

468 Ochi Y, Mukawa K, Kiyosawa K et al. Comparing the treatment outcomes of endoscopic papillary dilation and endoscopic sphincterotomy for removal of bile duct stones. J Gastroenterol Hepatol 1999; 14: 90-96

469 Arnold JC, Benz C, Martin WR et al. Endoscopic papillary balloon dilation vs. sphincterotomy for removal of common bile duct stones: a prospective randomized pilot study. Endoscopy 2001; 33: 563-567

470 Bergman JJ, van Berkel AM, Bruno MJ et al. A randomized trial of endoscopic balloon dilation and endoscopic sphincterotomy for removal of bile duct stones in patients with a prior Billroth II gastrectomy. Gastrointest Endosc 2001; 53: 19-26

471 Yasuda I, Tomita E, Enya $M$ et al. Can endoscopic papillary balloon dilation really preserve sphincter of Oddi function? Gut 2001; 49: 686-691

472 Natsui M, Narisawa R, Motoyama $\mathrm{H}$ et al. What is an appropriate indication for endoscopic papillary balloon dilation? Eur J Gastroenterol Hepatol 2002; 14: 635-640

473 Fujita N, Maguchi H, Komatsu Yet al. Endoscopic sphincterotomy and endoscopic papillary balloon dilatation for bile duct stones: A prospective randomized controlled multicenter trial. Gastrointest Endosc 2003; 57: 151-155

474 Vlavianos P, Chopra K, Mandalia S et al. Endoscopic balloon dilatation versus endoscopic sphincterotomy for the removal of bile duct stones: a prospective randomised trial. Gut 2003; 52: 1165-1169

475 DiSario JA, Freeman ML, Bjorkman DJ et al. Endoscopic balloon dilation compared with sphincterotomy for extraction of bile duct stones. Gastroenterology 2004; 127: 1291-1299

476 Lin CK, Lai KH, Chan HH et al. Endoscopic balloon dilatation is a safe method in the management of common bile duct stones. Dig Liver Dis 2004; 36: 68-72

477 Tanaka S, Sawayama T, Yoshioka T. Endoscopic papillary balloon dilation and endoscopic sphincterotomy for bile duct stones: long-term outcomes in a prospective randomized controlled trial. Gastrointest Endosc 2004; 59: 614-618

478 Weinberg BM, Shindy W, Lo S. Endoscopic balloon sphincter dilation (sphincteroplasty) versus sphincterotomy for common bile duct stones. Cochrane Database Syst Rev 2006; CD004890

479 Baron TH, Harewood GC. Endoscopic balloon dilation of the biliary sphincter compared to endoscopic biliary sphincterotomy for removal of common bile duct stones during ERCP: a metaanalysis of randomized, controlled trials. Am J Gastroenterol 2004; 99: 1455-1460

480 Chopra KB, Peters RA, PA OT et al. Randomised study of endoscopic biliary endoprosthesis versus duct clearance for bileduct stones in high-risk patients. Lancet 1996; 348: 791-793

481 Soomers AJ, Nagengast FM, Yap SH. Endoscopic placement of biliary endoprostheses in patients with endoscopically unextractable common bile duct stones. A long-term follow up study of 26 patients. Endoscopy 1990; 22: 24-26
482 Peters R, Macmathuna P, Lombard M et al. Management of common bile duct stones with a biliary endoprosthesis. Report on 40 cases. Gut 1992; 33: 1412-1415

483 Johnson GK, Geenen JE, Venu RP et al. Treatment of non-extractable common bile duct stones with combination ursodeoxycholic acid plus endoprostheses. Gastrointest Endosc 1993; 39: 528-531

484 Bergman JJ, Rauws EA, Tijssen JG et al. Biliary endoprostheses in elderly patients with endoscopically irretrievable common bile duct stones: report on 117 patients. Gastrointest Endosc 1995; 42: 195-201

485 Dalton HR, Chapman RW. Role of biliary stenting in the management of bile duct stones in the elderly. Gut 1995; 36: 485-487

486 Maxton DG, Tweedle DE, Martin DF. Retained common bile duct stones after endoscopic sphincterotomy: temporary and longterm treatment with biliary stenting. Gut 1995; 36: 446-449

487 Bowrey DJ, Fligelstone LJ, Solomon A et al. Common bile duct stenting for choledocholithiasis: a district general hospital experience. Postgrad Med J 1998; 74: 358-360

488 Jain SK, Stein R, Bhuva M et al. Pigtail stents: an alternative in the treatment of difficult bile duct stones. Gastrointest Endosc 2000; 52: $490-493$

489 Cotton PB. Stents for stones: short-term good, long-term uncertain. Gastrointest Endosc 1995; 42: 272-273

490 Hui CK, Lai KC, Ng M et al. Retained common bile duct stones: a comparison between biliary stenting and complete clearance of stones by electrohydraulic lithotripsy. Aliment Pharmacol Ther 2003; 17: 289-296

491 Boerma D, Rauws EA, Keulemans YC et al. Wait-and-see policy or laparoscopic cholecystectomy after endoscopic sphincterotomy for bile-duct stones: a randomised trial. Lancet 2002; 360: 761-765

492 Lau JY, Leow CK, Fung TM et al. Cholecystectomy or gallbladder in situ after endoscopic sphincterotomy and bile duct stone removal in Chinese patients. Gastroenterology 2006; 130: 96-103

493 Ando T, Tsuyuguchi T, Okugawa T et al. Risk factors for recurrent bile duct stones after endoscopic papillotomy. Gut 2003; 52: 116-121

494 Lee DW, Chan AC, Lam YH et al. Biliary decompression by nasobiliary catheter or biliary stent in acute suppurative cholangitis: a prospective randomized trial. Gastrointest Endosc 2002; 56: 361-365

495 Sharma BC, Kumar R, Agarwal $N$ et al. Endoscopic biliary drainage by nasobiliary drain or by stent placement in patients with acute cholangitis. Endoscopy 2005; 37: 439-443

496 Sauter G, Ruckdeschel G, Sauerbruch T. Antibiotische Prophylaxe und Therapie infektiöser Komplikationen bei der ERCP. Leber Magen Darm 1992; 22: 173-176

497 Niederau C, Pohlmann U, Lübke $H$ et al. Prophylactic antibiotic treatment in therapeutic or complicated diagnostic ERCP: results of a randomized controlled clinical study. Gastrointest Endosc 1994; 40: 533-537

498 Thompson BF, Arguedas MR, Wilcox CM. Antibiotic prophylaxis prior to endoscopic retrograde cholangiopancreatography in patients with obstructive jaundice: is it worth the cost? Aliment Pharmacol Ther 2002; 16: 727-734

499 Neoptolemos JP, Carr-Locke DL, London NJ et al. Controlled trial of urgent endoscopic retrograde cholangiopancreatography and endoscopic sphincterotomy versus conservative treatment for acute pancreatitis due to gallstones. Lancet 1988; 2: 979-983

500 Fan ST, Lai EC, Mok FP et al. Early treatment of acute biliary pancreatitis by endoscopic papillotomy. N Engl J Med 1993; 328: 228-232

501 Fölsch UR, Nitsche R, Lüdtke R et al. Early ERCP and papillotomy compared with conservative treatment for acute biliary pancreatitis. N Engl J Med 1997; 336: 237-242

502 Ayub K, Imada R, Slavin J. Endoscopic retrograde cholangiopancreatography in gallstone-associated acute pancreatitis. Cochrane Database Syst Rev 2004; CD003630

503 Oria A, Cimmino D, Ocampo C et al. Early endoscopic intervention versus early conservative management in patients with acute gallstone pancreatitis and biliopancreatic obstruction. A randomized clinical trial. Ann Surg 2007; 245: 10-17

504 Acosta JM, Katkhouda N, Debian KA et al. Early ductal decompression versus conservative management for gallstone pancreatitis with ampullary obstruction: a prospective randomized clinical trial. Ann Surg 2006; 243: 33-40

505 Bradley EL. A clinically based classification system for acute pancreatitis. Summary of the International Symposium on Acute Pancreatitis, Atlanta, GA, September 11 through 13,1992. Arch Surg 1993; 128 : $586-590$ 
506 Whitcomb DC. Acute Pancreatitis. N Engl J Med 2006; 354: $2142-2150$

507 Hammarström LE, Andersson $R$, Stridbeck $H$ et al. Influence of bile duct stones on patient features and effect of endoscopic sphincterotomy on early outcome of edematous gallstone pancreatitis. World J Surg 1999; 23: 12-17

508 De Waele B, Peterson T, Smekens L et al. Common bile duct stones in acute biliary pancreatitis: an endoscopic study. Surg Laparosc Endosc 1997; 7: 248-250

509 Arbuckle J, Isla A. Acute pancreatitis - update 2006. In: Neugebauer EA, Sauerland S, Fingerhut A, Millat B, Buess G (Hrsg). EAES Guidelines for Endoscopic Surgery. Twelve Years of Evidence-based Surgery in Europe. Berlin, Heidelberg: Springer, 2006: 377-386

510 Banks PA, Freeman ML. Practice guidelines in acute pancreatitis. Am J Gastroenterol 2006; 101: 2379-2400

511 Prat F, Edery J, Meduri B et al. Early EUS of the bile duct before endoscopic sphincterotomy for acute biliary pancreatitis. Gastrointest Endosc 2001; 54: 724-729

512 Hallal AH, Amortegui JD, Jeroukhimov IM et al. Magnetic resonance cholangiopancreatography accurately detects common bile duct stones in resolving gallstone pancreatitis. J Am Coll Surg 2005; 200: 869-875

513 Liu CL, Fan ST, Lo CM et al. Comparison of early endoscopic ultrasonography and endoscopic retrograde cholangiopancreatography in the management of acute biliary pancreatitis: a prospective randomized study. Clin Gastroenterol Hepatol 2005; 3: 1238-1244

514 Makary MA, Duncan MD, Harmon JW et al. The role of magnetic resonance cholangiography in the management of patients with gallstone pancreatitis. Ann Surg 2005; 241: 119-124

515 Kelly TR, Wagner DS. Gallstone pancreatitis: a prospective randomized trial of the timing of surgery. Surgery 1988; 104: 600-605

516 Nealon WH, Bawduniak J, Walser EM. Appropriate timing of cholecystectomy in patients who present with moderate to severe gallstoneassociated acute pancreatitis with peripancreatic fluid collections. Ann Surg 2004; 239: 741-749

517 Tate JJ, Lau WY, Li AK. Laparoscopic cholecystectomy for biliary pancreatitis. Br J Surg 1994; 81: 720-722

518 Uhl W, Müller CA, Krähenbühl L et al. Acute gallstone pancreatitis: timing of laparoscopic cholecystectomy in mild and severe disease. Surg Endosc 1999; 13: 1070-1076

519 Schachter P, Peleg T, Cohen O. Interval laparoscopic cholecystectomy in the management of acute biliary pancreatitis. HPB Surg 2000; 11 : 319-322

520 Schietroma M, Carlei F, Lezoche E et al. Acute biliary pancreatitis: staging and management. Hepatogastroenterology 2001; 48: 988-993

521 Taylor E, Wong $C$. The optimal timing of laparoscopic cholecystectomy in mild gallstone pancreatitis. Am Surg 2004; 70: 971-975

522 Cameron DR, Goodman AJ. Delayed cholecystectomy for gallstone pancreatitis: re-admissions and outcomes. Ann R Coll Surg Engl 2004; 86: 358-362

523 Hernandez V, Pascual I, Almela P et al. Recurrence of acute gallstone pancreatitis and relationship with cholecystectomy or endoscopic sphincterotomy. Am J Gastroenterol 2004; 99: 2417-2423

524 Gislason H, Vetrhus M, Horn A et al. Endoscopic sphincterotomy in acute gallstone pancreatitis: a prospective study of the late outcome. Eur J Surg 2001; 167: 204-208

525 Kaw M, Al-Antably Y, Kaw P. Management of gallstone pancreatitis: cholecystectomy or ERCP and endoscopic sphincterotomy. Gastrointest Endosc 2002; 56: 61-65

526 Hui CK, Lai KC, Yuen MF et al. The role of cholecystectomy in reducing recurrent gallstone pancreatitis. Endoscopy 2004; 36: 206-211

527 Ros E, Navarro S, Bru C et al. Occult microlithiasis in 'idiopathic' acute pancreatitis: prevention of relapses by cholecystectomy or ursodeoxycholic acid therapy. Gastroenterology 1991; 101: 1701-1709

528 Evans WB, Draganov P. Is empiric cholecystectomy a reasonable treatment option for idiopathic acute pancreatitis? Nat Clin Pract Gastroenterol Hepatol 2006; 3: 356-357

529 Garg PK, Tandon RK, Madan K. Is biliary microlithiasis a significant cause of idiopathic recurrent acute pancreatitis? A long-term follow-up study. Clin Gastroenterol Hepatol 2007; 5: 75-79

530 Testoni PA, Caporuscio S, Bagnolo F et al. Idiopathic recurrent pancreatitis: long-term results after ERCP, endoscopic sphincterotomy, or ursodeoxycholic acid treatment. Am J Gastroenterol 2000; 95: 1702-1707
531 Venneman NG, Renooij W, Rehfeld JF et al. Small gallstones, preserved gallbladder motility, and fast crystallization are associated with pancreatitis. Hepatology 2005; 41: 738-746

532 Venneman NG, Buskens E, Besselink MG et al. Small gallstones are associated with increased risk of acute pancreatitis: potential benefits of prophylactic cholecystectomy? Am J Gastroenterol 2005; 100: 2540-2550

533 Park DH, Kim MH, Lee SS et al. Accuracy of magnetic resonance cholangiopancreatography for locating hepatolithiasis and detecting accompanying biliary strictures. Endoscopy 2004; 36: 987-992

534 Mori T, Sugiyama M, Atomi Y. Gallstone disease: management of intrahepatic stones. Best Pract Res Clin Gastroenterol 2006; 20: 1117-1137

535 Kusano T, Isa T, Ohtsubo M et al. Natural progression of untreated hepatolithiasis that shows no clinical signs at its initial presentation. J Clin Gastroenterol 2001; 33: 114-117

536 Neuhaus $H$. Intrahepatic stones: the percutaneous approach. Can J Gastroenterol 1999; 13: 467-472

537 Di Carlo I, Sauvanet A, Belghiti J. Intrahepatic lithiasis: a Western experience. Surg Today 2000; 30: 319-322

538 Kim KH, Sung CK, Park BG et al. Clinical significance of intrahepatic biliary stricture in efficacy of hepatic resection for intrahepatic stones. J Hepatobiliary Pancreat Surg 1998; 5: 303-308

539 Adamek HE, Schneider AR, Adamek MU et al. Treatment of difficult intrahepatic stones by using extracorporeal and intracorporeal lithotripsy techniques: 10 years' experience in 55 patients. Scand J Gastroenterol 1999; 34: 1157-1161

540 Maetani I, Ishiguro J, Ogawa S et al. Percutaneous choledochoscopic treatment of intrahepatic stones, including management of associated biliary stenoses. Endoscopy 1999; 31: 456-459

541 Jeng KS, Sheen IS, Yang FS. Are modified procedures significantly better than conventional procedures in percutaneous transhepatic treatment for complicated right hepatolithiasis with intrahepatic biliary strictures? Scand J Gastroenterol 2002; 37: 597-601

542 Wu YW, Jian YP, Liang JS et al. The treatment of intrahepatic calculosis by applying helix hydro-jet lithotripsy under video choledochoscope: a report of 30 cases. Langenbecks Arch Surg 2006; 391: 355-358

543 Axelrad AM, Fleischer DE, Strack LL et al. Performance of ERCP for symptomatic choledocholithiasis during pregnancy: techniques to increase safety and improve patient management. Am J Gastroenterol 1994; 89: 109-112

544 Jamidar PA, Beck GJ, Hoffman BJ et al. Endoscopic retrograde cholangiopancreatography in pregnancy. Am J Gastroenterol 1995; 90: 1263-1267

545 Sungler P, Heinerman PM, Steiner $H$ et al. Laparoscopic cholecystectomy and interventional endoscopy for gallstone complications during pregnancy. Surg Endosc 2000; 14: 267-271

546 Tham TC, Vandervoort J, Wong RC et al. Safety of ERCP during pregnancy. Am J Gastroenterol 2003; 98: 308-311

547 Kahaleh M, Hartwell GD, Arseneau KO et al. Safety and efficacy of ERCP in pregnancy. Gastrointest Endosc 2004; 60: 287-292

548 Freistühler M, Braess A, Petrides AS. Ultraschallgeleitete endoskopische Papillotomie in der Schwangerschaft bei schwerer biliärer Pankreatitis. Z Gastroenterol 1999; 37: 27-30

549 Deutsche Gesellschaft für Medizinische Physik, Deutsche Röntgengesellschaft. Pränatale Strahlenexposition aus medizinischer Indikation. Dosisermittlung, Folgerungen für Arzt und Schwangere. Berlin: DGMP, 2002: 1-48

550 Testoni PA, Tittobello A. Long-term efficacy of endoscopic papillosphincterotomy for common bile duct stones and benign papillary stenosis. Surg Endosc 1991; 5: 135-139

551 Bergman JJ, van der Mey S, Rauws EA et al. Long-term follow-up after endoscopic sphincterotomy for bile duct stones in patients younger than 60 years of age. Gastrointest Endosc 1996; 44: 643-649

552 Lai KH, Peng NJ, Lo GH et al. Prediction of recurrent choledocholithiasis by quantitative cholescintigraphy in patients after endoscopic sphincterotomy. Gut 1997; 41: 399-403

553 Kratzer W, Mason RA, Grammer S et al. Difficult bile duct stone recurrence after endoscopy and extracorporeal shockwave lithotripsy. Hepatogastroenterology 1998; 45: 910-916

554 Tanaka M, Takahata S, Konomi H et al. Long-term consequence of endoscopic sphincterotomy for bile duct stones. Gastrointest Endosc 1998; 48: 465-469 
555 Sugiyama M, Suzuki Y, Abe N et al. Endoscopic retreatment of recurrent choledocholithiasis after sphincterotomy. Gut 2004; 53: 1856-1859

556 Swobodnik W, Janowitz P, Kratzer $W$ et al. Rezidivprophylaxe von Choledochuskonkrementen nach endoskopischer Papillotomie mit Ursodeoxycholsäure. Z Gastroenterol 1990; 28: 621-625

557 Paolucci V, Neckell M, Götze T. Das „okkulte“ Gallenblasenkarzinom CAE-S/CAMIC -Zentralregister. Zentralbl Chir 2003; 128: 309-312

558 Baron TH, Petersen BT, Mergener K et al. Quality indicators for endoscopic retrograde cholangiopancreatography. Am J Gastroenterol 2006; 101: 892-897

559 Freeman ML, Nelson DB, Sherman S et al. Complications of endoscopic biliary sphincterotomy. N Engl J Med 1996; 335: 909-918

560 Zinsser E, Hoffmann A, Will U et al. Erfolgs- und Komplikationsraten der diagnostischen und der therapeutischen endoskopischen retrograden Cholangiopankreatikografie - eine prospektive Studie. Z Gastroenterol 1999; 37: 707-713

561 Loperfido S, Angelini G, Benedetti $G$ et al. Major early complications from diagnostic and therapeutic ERCP: a prospective multicenter study. Gastrointest Endosc 1998; 48: 1-10

562 Freeman ML, DiSario JA, Nelson DB et al. Risk factors for post-ERCP pancreatitis: a prospective, multicenter study. Gastrointest Endosc 2001; 54: 425-434

563 Masci E, Toti G, Mariani A et al. Complications of diagnostic and therapeutic ERCP: a prospective multicenter study. Am J Gastroenterol 2001; 96: 417-423

564 Enns R, Eloubeidi MA, Mergener $K$ et al. ERCP-related perforations: risk factors and management. Endoscopy 2002; 34: 293-298

565 Christensen M, Matzen P, Schulze $S$ et al. Complications of ERCP: a prospective study. Gastrointest Endosc 2004; 60: 721-731

566 Washington M, Ghazi A. Complications of endoscopic retrograde cholangiopancreatography. In: Scott-Conner CE (Hrsg). The SAGES Manual. Fundamentals of Laparoscopy, Thoracoscopy, and GI Endoscopy. New York: Springer, 2006: 685-690

567 Vaira D, Dowsett JF, Hatfield AR et al. Is duodenal diverticulum a risk factor for sphincterotomy? Gut 1989; 30: 939-942

568 Mehta SN, Pavone E, Barkun JS et al. Predictors of post-ERCP complications in patients with suspected choledocholithiasis. Endoscopy 1998; 30: 457-463

569 Rabenstein T, Schneider HT, Bulling D et al. Analysis of the risk factors associated with endoscopic sphincterotomy techniques: preliminary results of a prospective study, with emphasis on the reduced risk of acute pancreatitis with low-dose anticoagulation treatment. Endoscopy 2000; 32: 10-19

570 Elfant $A B$, Bourke $M J$, Alhalel $R$ et al. A prospective study of the safety of endoscopic therapy for choledocholithiasis in an outpatient population. Am J Gastroenterol 1996; 91: 1499-1502

571 Freeman ML, Nelson DB, Sherman $S$ et al. Same-day discharge after endoscopic biliary sphincterotomy: observations from a prospective multicenter complication study. The Multicenter Endoscopic Sphincterotomy (MESH) Study Group. Gastrointest Endosc 1999; 49: $580-586$
572 Bittner R, Ulrich M. Gallenblasensteinleiden - immer eine Operationsindikation? Internist 2004; 45: 8-15

573 Eypasch E, Williams II, Wood-Dauphinee S et al. Gastrointestinal Quality of Life Index: development, validation and application of a new instrument. Br J Surg 1995; 82: 216-222

574 Eypasch E, Troidl H, Wood-Dauphinée S et al. Immediate improval in quality of life after laparoscopic cholecystectomy. Minim Invasive Ther Allied Technol 1993; 2: 139-146

575 Sanabria JR, Clavien PA, Cywes R et al. Laparoscopic versus open cholecystectomy: a matched study. Can J Surg 1993; 36: 330-336

576 Kane RL, Lurie N, Borbas C et al. The outcomes of elective laparoscopic and open cholecystectomies. J Am Coll Surg 1995; 180: 136-145

577 Plaisier PW. Incidence of persistent symptoms after laparoscopic cholecystectomy. Gut 1997; 41: 579

578 Mentes BB, Akin M, Irkorucu 0 et al. Gastrointestinal quality of life in patients with symptomatic or asymptomatic cholelithiasis before and after laparoscopic cholecystectomy. Surg Endosc 2001; 15: 1267-1272

579 Ludwig K, Patel K, Wilhelm L et al. Prospektive Analyse zur Outcomebewertung nach laparoskopischer versus konventioneller Cholecystektomie. Zentralbl Chir 2002; 127: 41-46

580 Topсu O, Karakayali F, Kuzu MA et al. Comparison of long-term quality of life after laparoscopic and open cholecystectomy. Surg Endosc 2003; 17: 291-295

581 Quintana JM, Arostegui I, Cabriada J et al. Predictors of improvement in health-related quality of life in patients undergoing cholecystectomy. Br J Surg 2003; 90: 1549-1555

582 Curet MJ, Contreras M, Weber DM et al. Laparoscopic cholecystectomy. Surg Endosc 2002; 16: 453-457

583 Keulemans Y, Eshuis J, de Haes H et al. Laparoscopic cholecystectomy: day-care versus clinical observation. Ann Surg 1998; 228: 734-740

584 Johansson M, Thune A, Nelvin L et al. Randomized clinical trial of daycare versus overnight-stay laparoscopic cholecystectomy. Br J Surg 2006; 93: 40-45

585 Lau H, Brooks DC. Transitions in laparoscopic cholecystectomy: the impact of ambulatory surgery. Surg Endosc 2002; 16: 323-326

586 Richardson WS, Fuhrman GS, Burch E et al. Outpatient laparoscopic cholecystectomy. Outcomes of 847 planned procedures. Surg Endosc 2001: 15: 193-195

587 Rosen MJ, Malm JA, Tarnoff $M$ et al. Cost-effectiveness of ambulatory laparoscopic cholecystectomy. Surg Laparosc Endosc Percutan Tech 2001; 11: 182-184

588 Maggiore $D$. Outpatient laparoscopic cholecystectomy: a reality. JSLS 2002; 6: 369-371

589 Feussner $H$. Laparoskopische Eingriffe. Möglichkeiten und Grenzen der ambulanten und kurzstationären Chirurgie. Chirurg 2004; 75 248-256

590 Lillemoe KD, Lin JW, Talamini MA et al. Laparoscopic cholecystectomy as a "true“ outpatient procedure: initial experience in 130 consecutive patients. J Gastrointest Surg 1999; 3: 44-49

591 Blatt $A$, Chen $S$. Day-only laparoscopic cholecystectomy in a regional teaching hospital. ANZ J Surg 2003; 73: 321-325 